Algorithms for

Psychometric Calculations

(Skeleton Tables for the

Thermodynamic Properties of Moist Air) 


\section{Announcing - The Building Science Series}

The "Building Science Series" disseminates technical information developed at the Bureau on building materials, components, systems, and whole structures. The series presents research results, test methods, and performance criteria related to the structural and environmental functions and the durability and safety characteristics of building elements and systems.

These publications, similar in style and content to the NBS Building Materials and Structure Reports (1938-59), are directed toward the manufacturing, design, and construction segments of the building industry, standards organizations, officials responsible for building codes, and scientists and engineers concerned with the properties of building materials.

The material for this series originates principally in the Building Research Division of the NBS Institute for Applied Technology. Published or in preparation are:

BSS1. Performance of Buildings - Concept and Measurement. Man and His Shelter. (In press.)

BSS2. Interrelations Between Cement and Concrete and Concrete Properties: Part 1, Materials and Techniques, W ater Requirements and Trace Elements. 35 cents

BSS3. Doors as Barriers to Fire and Smoke. 15 cents

BSS4. Weather Resistance of Porcelain EnameIs: Effect of Exposure Site and other Variables After Seven Years. 20 cents

BS55. Interrelations Between Cement and Concrete Properties: Part 2, Sulfate Expansion, Heat of Hydration, and Autoclave Expansion. 35 cents

BSS6. Some Properties of the Calcium Aluminoferrite Hydrates. 20 cents

BSS7. Organic Coatings. Properties, Selection, and Use. $\$ 2.50$

BSS8. Interrelations Between Cement and Concrete Properties: Part 3, Compressive Strengths of Portland Cement Test Mortars and Steam-Cured Mortars. 55 cents

BSS9. Thermal-Shock Resistance for Built. Up Membranes. 20 cents

BSS10. Field Burnout Tests of Apartment Dwelling Units. 25 cents

BSS11. Fire Resistance of Steel Deck Floor Assemblies. 25 cents

BSS12. Performance of Square-Edged Orifices and Orifice-Target Combinations as Air Mixers. 15 cents

BSS13. Shrinkage and Creep in Prestressed Concrete. 15 cents

BSS14. Experimental Determination of Eccentricity of Floor Loads Applied to a Bearing Wall. 15 cents

BSS15. Interrelations Between Cement and Concrete Properties: Part 4, Shrinkage of Hardened Portland Cement Pastes. 75 cents

BSS16. Techniques for the Survey and Evaluation of Live Floor Loads and Fire Loads in Modern Office Buildings. 40 cents

BSS17. Causes of Variations in Chemical Analysis and Physical Tests of Portland Cement. 40 cents

BSS18. Smoke and Gases Produced by Burning Aircraft Interior Materials. 35 cents

BSS19. Study of the Variables in the Saturating of Roofing Felts. 30 cents

Send orders with remittance to: Superintendent of Documents, U.S. Government Printing Office, Washington, D.C. 20402. Remittances from foreign countries should include an additional one-fourth of the purchase price for postage.

[See mailing list announcement on last page.] 
UNITED STATES DEPARTMENT OF COMMERCE • Maurice H. Stans, Secretary NATIONAL BUREAU OF STANDARDS $\bullet$ Lewis M. Branscomb, Director

\title{
Algorithms for Psychrometric Calculations (Skeleton Tables for the Thermodynamic Properties of Moist Air)
}

\author{
T. Kusuda \\ Building Research Division \\ Institute for Applied Technology \\ National Bureau of Standards \\ Washington, D.C., 20234
}

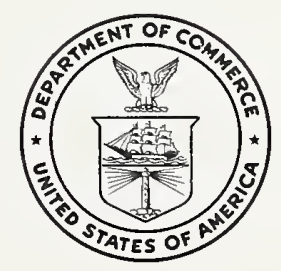

Building Science Series 21

Nat. Bur. Stand. (U.S.), Bldg. Sci. Ser. 21, 53 pages (Jan. 1970) CODEN: BSSNB

Issued Jan. 1970

For sale by the Superintendent of Documents, U.S. Government Printing Office Washington, D.C. 20402. (Order by SD Catalog No. C 13.29/2:21). Price 55 cents 
Library of Congress Catalog Card. No. 76-600863 


\section{Contents}

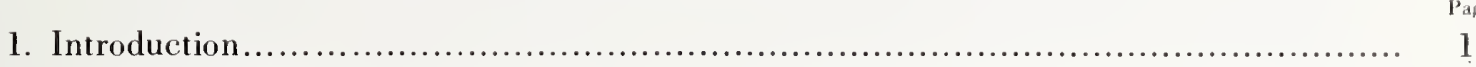

2. PSYCHR program .............................................................. 2

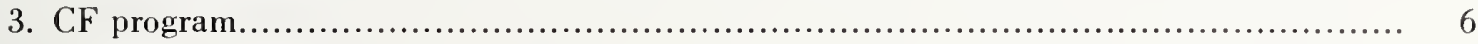

4. DBWBW program...................................................................... 7

5. DBDPW program..................................................................... 8

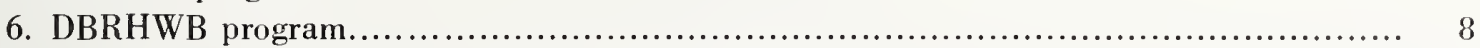

7. Comments on the new Kelvin temperature scale................................... 9

8. References......................................................................... 10

9. Sample results of psychrometric calculations..................................... 11 


\title{
Algorithms for Psychrometric Calculations (Skeleton Tables for the Thermodynamic Properties of Moist Air)
}

\section{T. Kusuda}

\begin{abstract}
Computer algorithms to obtain thermodynamic properties of saturated and unsaturated moist air are presented in this paper. The saturated moist air properties are calculated by the methodology developed by J. A. Goff and S. Gratch for their ASHRAE tables (1967 Book of Fundamentals, The American Society of Heating, Refrigerating and Air Conditioning Engineers). Sample calculations were performed using a computer program based upon the algorithms presented herein and the results are attached.

Key words: Computer algorithm; psychrometrics; saturated and unsaturated moist air; thermodynamic properties.
\end{abstract}

\section{Introduction}

Very accurate values of moist-air properties are required for many engineering problems. Most notable examples are those required in psychrometric calorimetry for measuring the capacity of various air conditioning apparatus, moisture transfer analyses in the cold storage warehouses, and analyses of simultaneous transfer of heat and moisture affecting the physiological responses of biological bodies. Although numerous psychrometric formulas and charts currently exist, the thermodynamic properties of moist air calculated by Goff and Gratch (1945) [1] ${ }^{1}$ are still considered most accurate and extensive. Their calculations are based upon the theory of statistical mechanics whereby interactions of major molecular components in the moist air are taken into consideration. Thus the calculation methodology employed by Goff and Gratch to produce the now well-known ASHRAE tables of moist-air properties should be valid beyond the ranges within which their calculations had been made. For example, the barometric pressure up to 3 atm and the temperature to $400 \mathrm{~K}$ can be covered. In addition, it is believed that the methodology is valid for a mole fraction composition of dry air different from that used in the original calculations.

Although the basic principle of calculation procedures for obtaining the moist air properties is described in the 1949 paper of J. A. Goff [2], it is not readily adaptable for machine calculation. The purpose of this paper is, then, to list step-by-step procedures for computer-oriented engineers to be able to calculate the accurate values of moist air properties based upon the Goff paper mentioned above. Since the paper is not intended for elaborating the thermodynamic principles inherent to the calculation procedures, those who wish to familarize themselves with that account should refer to references [1] and [2]. The complete National Bureau of Standards program called PSYCHR has been written to follow the calculation procedures described in this paper. This program has successfully reproduced the ASHRAE table of saturated moist air for 29.92 in of barometric pressure.

Additional programs called CF, DBWBW, DBDPW, DBRHWB, coupled with PSYCHR, can be used to calculate thermodynamic properties of unsaturated moist air by inputting values of the barometric pressure, dry-bulb temperature, and any one of several humidity indices such as wet-bulb temperature, dew-point temperature, relative humidity, and humidity ratio.

The program has been further employed to calculate the thermodynamic properties of moist air at various degrees of saturation and at various barometric pressure levels. Sample results of such calculations are attached to this algorithm. It is believed that the step-by-step account of the calculation methodology and the sample calculations presented herein should be very useful in future reference work relating to accurate psychrometric calculations.

1 Figures in brackets indicate the literature references on p. 10. 


\section{PSYCHR Program}

Input: $\quad P_{t}=$ barometric pressure (inches $\mathrm{Hg}$ )

$t=$ dry-bulb temperature $(\mathrm{F})$

Output: $h_{a}=$ enthalpy of dry air (Btu/lb of dry air)

$h_{s}=$ enthalpy of saturated moist air (Btu/lb of dry air)

$V_{a}=$ volume of dry air ( $\mathrm{ft}^{3} / \mathrm{lb}$ of dry air)

$V_{s}=$ volume of saturated moist air (ft. ${ }^{3} / \mathrm{lb}$ of dry air)

$W_{s}=$ humidity ratio of saturated moist air (lb of $\mathrm{H}_{2} \mathrm{O} / \mathrm{lb}$ of dry air)

$f_{s}=$ air-water interaction factor

$h_{w}=$ enthalpy of saturated water $\left(\mathrm{Btu} / \mathrm{lb}\right.$ of $\left.\mathrm{H}_{2} \mathrm{O}\right)$

$P_{v s}=$ vapor pressure of liquid water in moisture saturated air (inches $\mathbf{H g}$ )

$s_{a}=$ entropy of dry air $(\mathrm{Btu} / \mathrm{lb}, \mathrm{F})$

$s_{s}=$ entropy of saturated moist air (Btu/F, lb of dry air)

1. Composition of dry air.

Components

Chemical Symbols

Mole fraction $(X)$

Molecular weight $(M)$

(natural oxygen scale)

Molecular weight of air.

Molecular weight of water

\section{Oxygen}

$\mathrm{O} 2$

0.2095

32.000
Nitrogen
$\mathrm{N}_{2}$
0.7809
28.016

$M_{a}=28.966$
$M_{w}=18.016$

2. Kelvin temperature used for the calculations. ${ }^{2}$

$$
\begin{gathered}
T=\frac{t-32}{1.8}+273.16 \\
\tau=\frac{1}{T}
\end{gathered}
$$

\begin{tabular}{|c|c|c|c|c|c|}
\hline Components & Oxygen & Nitrogen & Argon & $\begin{array}{l}\text { Carbon } \\
\text { dioxide }\end{array}$ & Water \\
\hline C....................... & $7 / 2$ & $7 / 2$ & $5 / 2$ & $7 / 2$ & 4 \\
\hline$N_{1} \ldots \ldots \ldots \ldots \ldots \ldots \ldots$ & 1 & 1 & 0 & 2 & 1 \\
\hline$N_{2} \ldots \ldots \ldots \ldots \ldots \ldots \ldots$ & 0 & 0 & 0 & 1 & 1 \\
\hline$N_{3} \ldots \ldots \ldots \ldots \ldots \ldots$ & 0 & 0 & 0 & 1 & 1 \\
\hline$\theta_{1}(K) \ldots \ldots \ldots \ldots$ & 2235.4 & 3352.69 & $\infty$ & 960 & 2291.16 \\
\hline$\theta_{2}(K) \ldots \ldots$ & $\infty$ & $\infty$ & $\infty$ & 1944 & 5176.37 \\
\hline$\theta_{3}(K) \ldots \ldots \ldots \ldots \ldots$ & $\infty$ & $\infty$ & $\infty$ & 3379 & 5445.59 \\
\hline$A(K) \ldots \ldots \ldots \ldots \ldots \ldots$ & 1.073 & 0.9580 & 0 & 0 & 5.011 \\
\hline$B \ldots \ldots \ldots \ldots \ldots \ldots \ldots$ & 0 & 0.09 & 0 & 0 & 0 \\
\hline$D\left(K^{-1}\right) \ldots$ & $3.30 \times 10^{-6}$ & $2.023 \times 10^{-6}$ & 0 & 0 & $2.32 \times 10^{-5}$ \\
\hline$F \ldots \ldots$ & 0.011 & 0.009009 & 0 & 0 & 0 \\
\hline$a_{1} \ldots \ldots \ldots \ldots$ & 0 & 0 & 0 & 0 & -0.03958 \\
\hline$a_{2} \ldots \ldots \ldots \ldots \ldots \ldots \ldots$ & 0 & 0 & 0 & 0 & 0.05353 \\
\hline$a_{3} \ldots \ldots \ldots \ldots \ldots \ldots \ldots$ & 0 & 0 & 0 & 0 & 0.04000 \\
\hline Const................... & 1.2164 & -0.414686 & 1.867 & 1.8945 & -4.1083 \\
\hline
\end{tabular}

3. Zero pressure constants.

2If the new Kelvin Temperature Seale is to be used, 273.16 in this algorithm should be replaced by 273.15. Detailed discussions on this subject are presented beginning on p. 9 . 
4. For each component, calculate zero pressure enthalpy and entropy, $\frac{h^{\circ}}{R}$, and $\frac{s^{\circ}}{R}$ by the following relations.

$$
\begin{aligned}
\frac{h^{\circ}}{R} & =\frac{c}{\tau}+\sum_{k=1}^{3} \frac{N_{k} \theta_{k} e^{-\theta_{k} \tau}}{1-e^{-\theta_{k} \tau}}-A-2 B \tau+\frac{D}{\tau^{2}}+\sum_{k=1}^{3} a_{k} \theta_{k} e^{-\theta_{k} \tau}-\frac{F \theta_{1} e^{-\theta_{1 \tau}}}{\left(e^{-\theta_{1} \tau}-1\right)^{2}} \\
\frac{s^{\circ}}{R} & =c(1-\ln \tau)+\sum_{k=1}^{3} N_{k}\left\{\frac{\theta_{k} \tau-\left(1-e^{-\theta_{k} \tau}\right) \ln \left(1-e^{-\theta_{k} \tau}\right)}{\left(1-e^{-\theta_{k} \tau}\right)}\right\} \\
& -B \tau^{2}+\frac{2 D}{\tau}+\sum_{k=1}^{3} a_{k} e^{-\theta_{k} \tau}\left(\theta_{k} \tau+1\right)+F\left\{\frac{e^{\theta_{1 \tau}}\left(1-e^{\theta_{1 \tau} \tau}\right)-1}{\left(e^{\theta_{1 i}}-1\right)^{2}}\right\}+\text { Const }
\end{aligned}
$$

where $R=1.98583 \mathrm{cal} / \mathrm{mol}$, K.

5. Zero pressure enthalpy and entropy of dry air and water vapor are then obtained by

$$
\begin{aligned}
& h_{\mathrm{air}}^{\circ}=\left[\frac{X_{\mathrm{O}_{2}} h_{\mathrm{O}_{2}}^{\circ}+X_{\mathrm{N}_{2}} h_{\mathrm{N}_{2}}^{\circ}+X_{\mathrm{Ar}} h_{\mathrm{Ar}}^{\circ}+X_{\mathrm{CO}_{2}} h_{\mathrm{CO}_{2}}^{\circ}}{M_{a}}\right] \quad \mathrm{cal} / \mathrm{g} \\
& h_{\mathrm{H}_{2} \mathrm{O}}^{\circ} \leftarrow{\frac{h_{\mathrm{H}_{2} \mathrm{O}}^{\circ}}{M_{w}}}^{3} \quad \mathrm{cal} / \mathrm{g} \\
& s_{\text {air }}^{\circ}=\left[\frac{X_{\mathrm{O}_{2}} s_{\mathrm{O}_{2}}^{\circ}+X_{\mathrm{N}_{2}} S_{\mathrm{N}_{2}}^{\circ}+X_{\mathrm{Ar}} s_{\mathrm{Ar}}^{\circ}+X_{\mathrm{CO}_{2}} s_{\mathrm{CO}_{2}}^{\circ}}{M_{a}}\right]+0.03945 \quad \mathrm{cal} / \mathrm{g} \quad K \\
& s_{\mathrm{H}_{2} \mathrm{O}}^{\circ} \leftarrow \frac{s_{\mathrm{H}_{2} \mathrm{O}}^{\circ}}{M_{w}} \quad \mathrm{cal} / \mathrm{g} \quad \mathrm{K}
\end{aligned}
$$

6. Pressure in atmosphere

$$
P_{t} \leftarrow P_{t} / 29.921
$$

7. Virial coefficients:

$$
\begin{array}{rlr}
A_{a a}= & -40.70+13116 \tau+12 \tau^{3} \cdot 10^{7} & \\
B_{a a}= & -40.70+26232 \tau+48 \tau^{3} \cdot 10^{7} & \mathrm{~cm}^{3} / \mathrm{g} . \mathrm{mol} \\
C_{a a}= & \tau\left(A_{a a}-B_{a a}\right) & \mathrm{cm}^{3} / \mathrm{g} . \mathrm{mol} \\
A_{w w}=-33.97+55306 \tau \cdot 10^{72000 \tau^{2}} & \mathrm{~cm}^{3} / \mathrm{g} . \mathrm{mol}, \mathrm{K} \\
B_{w w}= & -33.97+110612 \tau \cdot 10^{72000 \tau^{2}} & \mathrm{~cm}^{3} / \mathrm{g} . \mathrm{mol} \\
& +55306 \tau^{2}\left(144000 \tau \cdot 10^{7200 \tau^{2}} \cdot \ln 10\right) & \mathrm{cm}^{3} / \mathrm{g} . \mathrm{mol} \\
C_{w w}= & \tau\left(A_{w w}-B_{w w}\right) & \\
A_{a w}= & -29.53+\frac{0.00669}{\tau}\left(1-e^{-\theta \tau}\right)+A \tau+B \tau^{2}+D \tau^{3} \mathrm{~cm}^{3} / \mathrm{g} \cdot \mathrm{mol}, \mathrm{K} \\
B_{a w}= & -29.53+0.00669 \theta e^{-\theta \tau}+2 A \tau+3 B \tau^{2}+4 D \tau^{3} & \mathrm{~cm}^{3} / \mathrm{g} . \mathrm{mol} \\
C_{a w}= & \tau\left(A_{a w}-B_{a w}\right) & \mathrm{cm}^{3} / \mathrm{g} . \mathrm{mol}, \mathrm{K} \\
& & \\
\text { where } A=17546 & \mathrm{~cm}^{3} \mathrm{~K} / \mathrm{g} . \mathrm{mol} \\
B=95300 & \mathrm{~cm}^{3} \mathrm{~K} / \mathrm{g} . \mathrm{mol} \\
D=8.515 \cdot 10^{2} & \mathrm{~cm}^{3} \mathrm{~K} / \mathrm{g} . \mathrm{mol} \\
\theta=4416.5 & \mathrm{~K}
\end{array}
$$

${ }^{3}$ Throughout this algorithm "๕" means "is replaced by" instead of "equals to" as in conventional mathematics. 


$$
\begin{aligned}
& A_{w w w}=0.0348 \tau^{2} A_{w w}^{3} \\
& B_{w w w}=0.1044 \tau^{2} A_{w w}^{2} B_{w w} \\
& C_{w w w}=\tau\left(A_{w w w}-B_{w w w}\right)
\end{aligned}
$$

8. Dry air properties

$$
\begin{aligned}
& h_{a}=1.8\left[h_{\text {air }}^{\circ}-\frac{0.0242179 \cdot B_{a a}\left(P_{t}\right)}{M_{a}}-60.99\right], \mathrm{Btu} / \mathrm{lb} \\
& s_{a}=s_{\text {air }}^{\circ}+\frac{0.0242179 \cdot C_{a a}\left(P_{t}\right)}{M_{a}}-\frac{R}{M_{a}} \ln \left(P_{t}\right)-1.60096, \mathrm{Btu} / \mathrm{lb}, \mathrm{F} \\
& V_{a}=\left(\frac{453.5924}{28316.85}\right) \frac{\left(\frac{82.0567}{P_{t}}-A_{a t a}\right)}{M_{a}}, \mathrm{ft}^{3} / \mathrm{lb}
\end{aligned}
$$

9. Calculate the water vapor pressure. If $T \leqslant 273.16$,

$$
\begin{aligned}
u & =273.16 \tau \\
z & =-9.09718(u-1)-3.56654 \log _{10} u+0.876793\left(1-\frac{1}{u}\right) \\
P_{s} & =0.0060273 \cdot 10^{z} \ldots(\mathrm{atm}) \ldots . \text { vapor pressure of ice. }
\end{aligned}
$$

if $T>273.16$,

$$
\begin{aligned}
u= & 373.16 \tau \\
z= & -7.90298(u-1)+5.02808 \log _{10} u \\
& -1.3816 \cdot 10^{-7}\left\{10^{11.344(1-1 / u)}-1\right\} \\
& +8.1328 \cdot 10^{-3}\left(10^{-3.49149(u-1)}-1\right) \\
P_{s}= & 10^{z} \ldots \text { atm } \ldots \text { vapor pressure of liquid water. } \\
P_{v s}= & 29.921 \cdot P_{s}
\end{aligned}
$$

10. Calculate humidity ratio and mole fraction of moisture saturated air.

$$
\begin{aligned}
\alpha & =\left(A_{a a}-2 A_{a w}+A_{w w}\right) \frac{P_{s} \tau}{82.0567}+\frac{A w w w P_{s}^{2} \tau}{82.0567} \\
z^{\prime} & =\alpha\left(1-\frac{P_{s}}{P_{t}}\right)+\beta\left(\frac{P_{t}}{P_{s}}-1\right) \quad(\beta \text { is tabulated below }) \\
f_{s} & =e^{z^{\prime}} \\
W_{s} & =0.62197 \frac{f_{s} \frac{P_{s}}{P_{t}}}{1-f_{s} \frac{P_{s}}{P_{t}}} \\
Y_{s} & =\frac{18.016}{28.966 \cdot W_{s}+18.016}
\end{aligned}
$$


11. Table of $\beta, h^{\prime}, L$ for the Lagrangean interpolation.

\begin{tabular}{|c|c|c|}
\hline$\left(\frac{t-32}{1.8}\right)$ & $\beta^{*}$ & $h_{w}^{\prime}(\mathrm{cal} / \mathrm{g})$ \\
\hline-80 & $0.44 * 10^{-8}$ & -114.25 \\
\hline-70 & $1.90 * 10^{-8}$ & -110.54 \\
\hline-60 & $0.71 * 10^{-7}$ & -106.64 \\
\hline-50 & $2.35^{*} 10^{-7}$ & -102.58 \\
\hline-40 & $0.70^{*} 10^{-6}$ & -98.34 \\
\hline-30 & $1.91 * 10^{-6}$ & -93.92 \\
\hline-20 & $0.48^{*} 10^{-5}$ & -89.34 \\
\hline-10 & $1.11^{*} 10^{-5}$ & -84.57 \\
\hline 0 & $2.43^{*} 10^{-5}$ & -79.64 \\
\hline 0 & $2.37^{*} 10^{-5}$ & 0.02 \\
\hline 10 & $4.44^{*} 10^{-5}$ & 10.06 \\
\hline 20 & $0.79 * 10^{-4}$ & 20.06 \\
\hline 30 & $1.34^{*} 10^{-4}$ & 30.04 \\
\hline 40 & $2.19^{*} 10^{-4}$ & 40.03 \\
\hline 50 & $3.46^{*} 10^{-4}$ & 50.01 \\
\hline 60 & $5.26^{*} 10^{-4}$ & 60.00 \\
\hline 70 & $0.78^{*} 10^{-3}$ & 69.99 \\
\hline 80 & $1.12^{*} 10^{-3}$ & 80.01 \\
\hline 90 & $1.58 * 10^{-3}$ & 90.05 \\
\hline
\end{tabular}

12. Calculate the properties of water vapor.

$$
\begin{aligned}
h_{g}^{\prime}= & h_{H_{2} O}^{\circ}-\frac{0.0242179}{M_{w}}\left\{B_{w w} P_{s}+\frac{1}{2} B_{w w w} P_{s}^{2}\right\}+477.277 \mathrm{cal} / \mathrm{g} \\
h_{g}= & 1.8 h_{g}^{\prime} \ldots . \mathrm{Btu} / \mathrm{lb} \\
s_{y}= & s_{H_{2} O}^{\circ}+\frac{0.0242179}{M_{w}}\left\{C_{w w} P_{s}+\frac{1}{2} C_{w w w} P_{s}^{2}\right\} \\
& -\frac{\mathrm{R}}{M_{w}} \ln P_{s}-0.83960, \mathrm{Btu} / \mathrm{lb}, \mathrm{F} \text { or } \mathrm{cal} / \mathrm{g}, \mathrm{C} \\
V_{g}= & \frac{1}{M_{w}}\left(\frac{453.5924}{28316.85}\right)\left(\frac{82.0567}{P_{s} \tau}-A_{w w}-A_{w w u} P_{s}\right), \mathrm{ft}^{3} / \mathrm{lb}
\end{aligned}
$$

13. Enthalpy of liquid water.

$$
h_{w}=1.8 h_{w}^{\prime} \ldots . \mathrm{Btu} / \mathrm{lb}
$$


14. Calculate the properties of moist air 'saturated with water vapor.

$$
\begin{aligned}
h_{s}= & \frac{1.8}{M_{a} Y_{s}}\left\{\left[M_{a} Y_{s} h_{\mathrm{air}}^{\circ}+M_{w}\left(1-Y_{s}\right) h_{\mathrm{H}_{2} \mathrm{O}}^{\circ}\right]\right. \\
& -0.0242179\left[\left(Y_{s}^{2} \cdot B_{a a}+2 Y_{s}\left(1-Y_{s}\right) B_{a w}+\left(1-Y_{s}\right)^{2} B_{w w}\right) P_{t}\right. \\
& \left.\left.-1 / 2\left(1-Y_{s}\right)^{3} B_{w w w} P_{t}^{2}\right]\right\}-109.782+859.099 W_{s}, \text { B.t.u./lb. of dry air } \\
s_{s}= & \frac{1}{M_{a} Y_{s}}\left\{\left[M_{a} Y_{s} s_{\mathrm{air}}^{\circ}+M_{w}\left(1-Y_{s}\right) s_{\mathrm{H}_{2} \mathrm{O}}^{\circ}\right]\right. \\
& +0.0242179\left[\left(Y_{s}^{2} C_{a a}+2 Y_{s}\left(1-Y_{s}\right) C_{a w}+\left(1-Y_{s}\right)^{2} C_{w w}\right) P_{t}\right. \\
& \left.+1 / 2\left(1-Y_{s}\right)^{3} C_{w w w} P_{t}^{2}\right]-R\left[Y_{s} \ln Y_{s}+\left(1-Y_{s}\right) \ln \left(1-Y_{s}\right)+\ln P_{t}\right] \\
& -1.60096-0.83960 W_{s}, \text { B.t.u./lb. of dry air, }{ }^{\circ} \mathrm{R} \\
V_{s}= & \frac{1}{M_{a} Y_{s}}\left(\frac{453.5924}{28316.85}\right)\left\{\frac{82.0567}{P_{t} \tau}\right. \\
& -\left[\left(Y_{s}^{2} A_{a a}+2 Y_{s}\left(1-Y_{s}\right) A_{a w}+\left(1-Y_{s}\right)^{2} A_{w w}\right)\right. \\
& \left.\left.-\left(1-Y_{s}\right)^{3} A_{w w w} P_{t}\right]\right\} \mathrm{ft} 3 / \mathrm{lb} \text { of dry air }
\end{aligned}
$$

\section{CF Program}

Comments: When $t \geqslant 112^{\circ} \mathrm{F}$, this program CF is used to find correction terms for $h$ and $v$ calculated by

$$
h=h_{a}+\left(W / W_{s}\right)\left(h_{s}-h_{a}\right), V=V_{a}+\left(W / W_{s}\right)\left(V_{s}-V_{a}\right) .
$$

Input: $t=$ dry bulb temperatures $(\mathrm{F})$

$W=$ humidity ratio $\left(\mathrm{lb} \mathrm{H}_{2} \mathrm{O} / \mathrm{lb}\right.$ of dry air)

$W_{s}=$ humidity ratio of saturated moist air $\left(\mathrm{lb} \mathrm{H}_{2} \mathrm{O} / \mathrm{lb}\right.$ of dry air)

Output: $\bar{V}=$ correction terms to the volume $v$ (ft/lb of dry air)

$\bar{h}=$ correction term to the enthalpy $h$ (Btu/lb of dry air)

Calculation procedures:

1. Use the Lagrangean interpolation technique to pick up $A$ and $B$ from the following table:

\begin{tabular}{r|r|r}
\hline \hline \multicolumn{1}{c|}{$t$} & $A$ & $B$ \\
\hline 96 & 0.0018 & 0.0268 \\
112 & .0042 & .0650 \\
128 & .0096 & .1439 \\
144 & .0215 & .3149 \\
160 & .0487 & .6969 \\
176 & .1169 & 1.636 \\
192 & .3363 & 4.608 \\
\hline
\end{tabular}

2. $\bar{v}=\frac{\left(\frac{W}{W_{s}}\right) \cdot\left(1-\frac{W}{W_{s}}\right) A}{1+1.6078 W}$

3. $\vec{h}=\left(\frac{W}{W_{s}}\right)\left(1-\frac{W}{W_{s}}\right) B /(1+1.6078 W)$ 


\section{DBWBW Program}

Input: $t=\mathrm{dry}$-bulb temperature $(\mathrm{F})$

$t^{*}=$ thermodynamic wet-bulb temperature $(\mathrm{F})$

$P_{t}=$ barometric pressure (inches $\mathrm{Hg}$ )

Output: $W=$ humidity ratio of moist air (lb of $\mathrm{H}_{2} \mathrm{O} / \mathrm{lb}$ of dry air)

$h=$ enthalpy of moist air (Btu/lb of dry air)

$V=$ volume of moist air $\left(\mathrm{ft}^{3} / \mathrm{lb}\right.$ of dry air)

$\mu=$ degree of saturation

$P_{v}=$ water vapor pressure, inches $\mathrm{Hg}$

$D P=$ dew-point temperature, $\mathrm{F}$

$R H=$ relative humidity, \%

Calculation procedures:

1. CALL PSYCHR $\left(P_{t}, P_{v s}, t, h_{a}, h_{s}, V_{a}, V_{s}, W_{s}, f_{s}, h_{w}\right)$

2. CALL PSYCHR $\left(P_{t}, P_{v s}, t^{*}, h_{a}^{*}, h_{s}^{*}, V_{a}^{*}, V_{s}^{*}, W_{s}^{*}, f_{s}^{*}, h_{w}^{*}\right)^{4}$

3. Iterate on $W$ to satisfy

where $\bar{h}$ is obtained by

$$
\begin{aligned}
& h=h_{a}+\frac{W}{W_{s}}\left(h_{s}-h_{a}\right)+\bar{h} \\
& h+\left(W_{s}^{*}-W\right) h_{w}^{*}=h_{s}^{*}
\end{aligned}
$$

$\operatorname{CALL~} \mathrm{CF}\left(t, W, W_{s}, \bar{V}, \bar{h}\right)$.

If $t \leqslant 112^{\circ} \mathrm{F}$, however, $W$ may be calculated by

4. If $t \leqslant 112^{\circ} \mathrm{F}$,

$$
\begin{gathered}
W=\left[\frac{h_{s}^{*}-h_{a}-h_{w}^{*} W_{s}^{*}}{h_{s}-h_{a}-h_{w}^{*} W_{s}}\right] \cdot W_{s} . \\
h=h_{a}+\frac{W}{W_{s}}\left(h_{s}-h_{a}\right) \\
V=V_{a}+\frac{W}{W_{s}}\left(V_{s}-V_{a}\right) .
\end{gathered}
$$

5. If $t>112^{\circ} \mathrm{F}$,

CALL CF $\left(t, W, W_{s,}, \bar{V}, \bar{h}\right)$

$$
\begin{gathered}
h \leftarrow h+\bar{h} \\
V \leftarrow V+\bar{V} \\
\mu=\frac{W}{W_{s}} .
\end{gathered}
$$

6.

7.

$$
R H=\frac{\mu \times 100}{1-(1-\mu) f_{s} \frac{P_{v s}}{P_{t}}}
$$

8. Iterate PSYCHR on various DP until

$$
W=W_{s}(D P) .
$$

9.

$$
P V=P_{v s}(D P)
$$




\section{DBDPW Program}

Input: $t=\mathrm{dry}-\mathrm{bulb}$ temperature $(\mathrm{F})$

$D P=$ dew-point temperature $(\mathrm{F})$

$P_{t}=$ barometric pressure (inches $\mathrm{Hg}$ )

Output: $W=$ humidity ratio (lb of $\mathrm{H}_{2} \mathrm{O} / \mathrm{lb}$ of dry air)

$h=$ enthalpy (Btu/lb of dry air)

$V=$ volume $\left(\mathrm{ft}^{3} / \mathrm{lb}\right.$ of dry air)

$R H=$ relative humidity, $\%$

$t^{*}=$ thermodynamic wet-bulb temperature, $\mathrm{F}$

Calculation procedures:

1. CALL PSYCHR $\left(P_{t}, P_{v s}, D P, h_{a}^{\prime}, h_{s}^{\prime}, V_{a}^{\prime}, V_{s}^{\prime}, W_{s}^{\prime}, f_{s}^{\prime}, h_{w}^{\prime}\right)$

$$
W=W_{s}^{\prime}
$$

2. CALL PSYCHR $\left(P_{t}, P_{t s}, t, h_{a}, h_{s}: V_{a}, V_{s}, W_{s}, f_{s}, h_{w}\right)$

3. If $t \leqslant 112^{\circ} \mathrm{F}$

4. If $t>112^{\circ} \mathrm{F}$

$$
h=h_{a}+\frac{W}{W_{s}}\left(V_{s}-V_{a}\right) .
$$

$\operatorname{CALL~CF~}\left(t, W, W_{s}, \bar{V}, \bar{h}\right)$

$$
\begin{aligned}
& h=h_{a}+\frac{W}{W_{s}}\left(h_{s}-h_{a}\right)+\bar{h} \\
& V=V_{a}+\frac{W}{W_{s}}\left(V_{s}-V_{a}\right)+\bar{V}
\end{aligned}
$$

5.

$$
\begin{aligned}
\mu & =\frac{W}{W_{s}} \\
R H & =\frac{\mu \times 100}{1-(1-\mu) f_{s} \frac{P_{v s}}{P_{t}}}
\end{aligned}
$$

6. Iterate $t^{*}$ on the following equation by making use of PSYCHR.

$$
h+\left(W_{s}\left(t^{*}\right)-W\right) h_{w}\left(t^{*}\right)=h_{s}\left(t^{*}\right) .
$$

\section{DBRHWB Program}

Input: $P_{t}=$ barometric pressure (inches $\mathrm{Hg}$ )

$t=$ dry-bulb temperature $(\mathrm{F})$

$\phi=$ relative humidity, fraction

Output: $t^{*}=$ thermodynamic wet-bulb temperature $(\mathrm{F})$

Calculation procedures:

1. CALL PSYCHR $\left(P_{t}, P_{v s}, t, h_{a}, h_{s}, \bar{V}_{a}, V_{s}, W_{s}, f_{s}, h_{w}\right)$

$$
P_{s}=\phi P_{v s} \quad \text { approximation) }
$$

3.

$$
\mu=\frac{\phi\left(1-f_{s} \frac{P_{s}}{P_{t}}\right)}{1-\phi f_{s} \frac{P_{s}}{P_{\imath}}} .
$$


If $t \leqslant 112^{\circ} \mathrm{F}$

If $t>112^{\circ} \mathrm{F}$

$$
h=h_{a}+\mu\left(h_{s}-h_{a}\right) .
$$

$\operatorname{CALL~CF}\left(t, W, W_{s}, \bar{V}, \bar{h}\right)$

$$
h \leftarrow h+\bar{h}
$$

4.

$$
W=\mu W_{s}
$$

5. Iterate the following formula to find $t^{*}$ by making use of PSYCHR.

$$
h+\left(W_{s}\left(t^{*}\right)-W\right) \cdot h_{w}\left(t^{*}\right)=h_{s}\left(t^{*}\right)
$$

\section{Comments on the New Kelvin Temperature Scale}

The values for saturation pressure of water calculated by the algorithms presented herein are identical with those tabulated in table 2 of Chapter 21 of 1967 ASHRAE Book of Fundamentals. These values were, however, obtained by the Goff's calculation based upon the old Kelvin scale $[t=T-273.16$ (Centigrade vs. Kelvin)]. In October 1954, the Tenth General Conference on Weights and Measures adopted a new Kelvin scale $T$ of absolute temperature on which the triplepoint of water is assigned the value 273.16. According to this new convention, the new temperature scale becomes $t=T-273.15$. Prof. J. A. Goff published new formulas corrected for this new temperature scale for saturation pressure in his most recent paper (Saturation Pressure of Water on the New Kelvin Temperature Scale, 1963 International Symposium on Humidity and Moisture, Washington, D.C.). According to that paper, the saturation pressure for the new temperature scale will be calculated by the following algorithms.

$$
\begin{gathered}
u=273.16 /[(t-32) / 1.8+273.15] \\
P_{s}=29.9210^{z}
\end{gathered}
$$

when (1) saturation over ice

$$
\begin{aligned}
z= & -9.096936(u-1)-3.56654 \log _{10} u \\
& +0.876817\left(1-\frac{1}{u}\right)-2.2195983
\end{aligned}
$$

(2) saturation over liquid water

$$
\begin{aligned}
z= & -10.79586(u-1)-5.02808 \log _{10} u \\
& +1.50474 \cdot 10^{-4}\left(1-10^{-8 \cdot 29692(1 / u-)}\right) \\
& +0.42873 \cdot 10^{-3}\left(10^{4 \cdot 76955(1-u)}-1\right) \\
& -2.2195983
\end{aligned}
$$

According to this new formula, the corrections $\triangle P / P$ to the current ASHRAE table values of the saturation water vapor pressure are found to be extremely small, as shown in the following table. 


\begin{tabular}{lll} 
over ice & $t\left({ }^{\circ} \mathrm{F}\right)$ & $\Delta P / P$ \\
& -292 & -0.00513 \\
-220 & -0.00216 \\
-148 & -0.001050 \\
-76 & -0.000541 \\
& -4 & -0.000269 \\
& +32 & -0.000181 \\
\hline over liquid & 32 & -0.000193 \\
water & 68 & -0.0001320 \\
& 104 & -0.0000854 \\
& 140 & -0.0000493 \\
& 176 & -0.0000212 \\
& 212 & 0.00000
\end{tabular}

For the engineering calculation, the algorithms based upon Goff's 1949 paper for PSYCHR should, therefore, be sufficiently accurate.

\section{Unit Conversion Table}

\begin{tabular}{|c|c|c|c|}
\hline$V$ & $\begin{array}{l}\text { To Convert From } \\
{\left[\mathrm{ft}^{3} / \mathrm{lb}\right]}\end{array}$ & $V^{\prime}\left[\mathrm{m}^{3} / \mathrm{kg}\right]$ & $\begin{array}{l}\text { Operation Required } \\
\dot{V}^{\prime}=0.06243 \dot{V}\end{array}$ \\
\hline$P$ & {$\left[\begin{array}{c}\text { in } \mathrm{Hg}^{*} \\
\left(32{ }^{\circ} \mathrm{F}\right)\end{array}\right]$} & $P^{\prime}\left[\mathrm{N} / \mathrm{m}^{2}\right]$ & $P^{\prime}=3386.389 P$ \\
\hline$h$ & {$[\mathrm{Btu} / \mathrm{lb}]$} & $\begin{array}{l}P^{\prime \prime}\left[\mathrm{kg} / \mathrm{m}^{2}\right] \\
P^{\prime \prime \prime}[\mathrm{mm} \mathrm{Hg}]\end{array}$ & $\begin{aligned} P^{\prime \prime} & =345.3 P \\
P^{\prime \prime \prime} & =25.4 P \\
h^{\prime} & =(h-7686) 2326\end{aligned}$ \\
\hline & & $h^{\prime \prime}[\mathrm{kcal} / \mathrm{kg}]$ & $h^{\prime \prime}=\left(\frac{h-7.686}{1.8}\right)$ \\
\hline$s$ & {$\left[\mathrm{Btu} / \mathrm{lb}{ }^{\circ} \mathrm{R}\right]$} & $\begin{array}{ll}s^{\prime} & {[\mathrm{kcal} / \mathrm{kg} \mathrm{K}]} \\
s^{\prime \prime} & {[\mathrm{J} / \mathrm{kg} \mathrm{K}]}\end{array}$ & $\begin{array}{l}s^{\prime}=s-0.01617 \\
s^{\prime \prime}=(s-0.01617) 4184\end{array}$ \\
\hline$W$ & {$[\mathrm{lb} / \mathrm{lb}]$} & $W^{\prime}[g / \mathrm{kg}]$ & $W^{\prime}=1000 W$ \\
\hline
\end{tabular}

*Density, $13.595 \mathrm{~g} / \mathrm{cm}^{3}$

\section{References}

[1] Goff, J. A., and Gratch, S., Thermodynamic properties of moist air, ASHVE Transaction, 1945, pp. $125-164$.

[2] Goff, J. A., Standardization of thermodynamic properties of moist air, ASHVE Journal Section, HPAC, Nov. 1949. 
9. Sample Results of Psychrometric Calculations 


\title{
NOMENCLATURE
}

\author{
ALT............ Altitude, $\mathrm{ft}$ \\ DB............. Dry-bulb temperature, F \\ WB........... Thermodynamic wet-bulb temperature, $\mathrm{F}$ \\ DP............ Dewpoint temperature, $\mathrm{F}$ \\ RH ............ Relative humidity, percent \\ PV ............. Vapor pressure, in. hg \\ W.............. Humidity ratio \\ H............. Enthalpy, Btu per lb of dry air \\ S ............. Entropy, Btu per F per lb of dry air \\ V............... Volume, cu ft per lb of dry air \\ PB............ Barometric pressure, in. hg
}

Thermodynamic properties tabulated in this publication are calculated by the Goff and Gratch formulas originally published in Standardization of Thermodynamic Properties of Moist Air (ASHVE Journal Section 1949). 
$\mathrm{PB}=31.02$, Altitude $=-1000$

\begin{tabular}{|c|c|c|c|c|c|c|c|c|}
\hline $\mathrm{DB}$ & WB & $\mathrm{DP}$ & $\mathrm{RH}$ & PV & W & $\mathrm{H}$ & S & V \\
\hline-40.0 & -40.0 & -40.0 & 100.0 & .0038 & .00008 & -9.54 & -.0242 & 10.19 \\
\hline .0 & .0 & .0 & 100.0 & .0376 & .00076 & .80 & -.0006 & 11.18 \\
\hline .0 & -.1 & -.6 & 96.9 & .0365 & .00074 & .77 & -.0007 & 11.18 \\
\hline .0 & -.2 & -1.2 & 93.8 & .0353 & .00071 & .75 & -.0007 & 11.18 \\
\hline .0 & -.3 & -1.9 & 90.7 & .0341 & .00069 & .72 & -.0008 & 11.18 \\
\hline .0 & -.4 & -2.5 & 87.5 & .0330 & .00066 & .70 & -.0008 & 11.18 \\
\hline .0 & -.5 & -3.2 & 84.4 & .0318 & .00064 & .67 & -.0009 & 11.18 \\
\hline .0 & -.6 & -3.9 & 81.3 & .0306 & .00062 & .65 & -.0010 & 11.18 \\
\hline .0 & -.7 & -4.6 & 78.2 & .0294 & .00059 & .62 & -.0010 & 11.18 \\
\hline .0 & -.8 & -5.4 & 75.1 & .0283 & .00057 & .60 & -.0011 & 11.18 \\
\hline .0 & -.9 & -6.2 & 72.0 & .0271 & .00055 & .57 & -.0011 & 11.18 \\
\hline .0 & -1.0 & -7.0 & 68.9 & .0259 & .00052 & .55 & -.0012 & 11.18 \\
\hline .0 & -1.1 & -7.8 & 65.8 & .0248 & .00050 & .52 & -.0012 & 11.18 \\
\hline .0 & -1.2 & -8.7 & 62.7 & .0236 & .00048 & .50 & -.0013 & 11.18 \\
\hline .0 & -1.3 & -9.7 & 59.7 & .0225 & .00045 & .47 & -.0013 & 11.18 \\
\hline .0 & -1.4 & -10.6 & 56.6 & .0213 & .00043 & .45 & -.0014 & 11.18 \\
\hline .0 & -1.5 & -11.6 & 53.5 & .0201 & .00041 & .42 & -.0015 & 11.18 \\
\hline .0 & -1.6 & -12.7 & 50.4 & .0190 & .00038 & .40 & -.0015 & 11.17 \\
\hline .0 & -1.7 & -13.8 & 47.3 & .0178 & .00036 & .37 & -.0016 & 11.17 \\
\hline .0 & -1.8 & -15.0 & 44.3 & .0167 & .00034 & .35 & -.0016 & 11.17 \\
\hline .0 & -1.9 & -16.3 & 41.2 & .0155 & .00031 & .32 & -.0017 & 11.17 \\
\hline .0 & -2.0 & -17.7 & 38.1 & .0143 & .00029 & .30 & -.0017 & 11.17 \\
\hline .0 & -2.1 & -19.2 & 35.0 & .0132 & .00027 & .28 & -.0018 & 11.17 \\
\hline .0 & -2.2 & -20.8 & 32.0 & .0120 & .00024 & .25 & -.0019 & 11.17 \\
\hline .0 & -2.3 & -22.5 & 28.9 & .0109 & .00022 & .23 & -.0019 & 11.17 \\
\hline .0 & -2.4 & -24.4 & 25.8 & .0097 & .00020 & .20 & -.0020 & 11.17 \\
\hline 40.0 & 40.0 & 40.0 & 100.0 & .2477 & .00503 & 15.02 & .0290 & 12.24 \\
\hline 40.0 & 39.0 & 37.8 & 91.7 & .2271 & .00461 & 14.57 & .0281 & 12.23 \\
\hline 40.0 & 38.0 & 35.4 & 83.5 & .2069 & .00420 & 14.13 & .0272 & 12.23 \\
\hline 40.0 & 37.0 & 32.9 & 75.5 & .1870 & .00379 & 13.69 & .0263 & 12.22 \\
\hline 40.0 & 36.0 & 30.4 & 67.6 & .1674 & .00339 & 13.26 & .0254 & 12.21 \\
\hline 40.0 & 35.0 & 27.7 & 59.8 & .1481 & .00300 & 12.84 & .0245 & 12.20 \\
\hline 40.0 & 34.0 & 24.8 & 52.1 & .1291 & .00261 & 12.42 & .0237 & 12.19 \\
\hline 40.0 & 33.0 & 21.5 & 44.6 & .1104 & .00223 & 12.01 & .0228 & 12.19 \\
\hline 40.0 & 32.0 & 19.9 & 41.3 & .1024 & .00207 & 11.83 & .0225 & 12.18 \\
\hline 40.0 & 31.0 & 16.0 & 34.2 & .0846 & .00171 & 11.45 & .0216 & 12.18 \\
\hline 40.0 & 30.0 & 11.3 & 27.1 & .0671 & .00135 & 11.06 & .0208 & 12.17 \\
\hline 40.0 & 29.0 & 5.4 & 20.1 & .0499 & .00101 & 10.69 & .0200 & 12.16 \\
\hline 40.0 & 28.0 & -2.5 & 13.3 & .0330 & .00067 & 10.32 & .0192 & 12.16 \\
\hline 40.0 & 27.0 & -15.3 & 6.6 & .0164 & .00033 & 9.96 & .0184 & 12.15 \\
\hline 40.0 & 26.0 & -85.5 & .1 & .0002 & .00000 & 9.61 & .0176 & 12.14 \\
\hline
\end{tabular}


$\mathrm{PB}=31.02$, Altitude $=-1000-$ Continued

\begin{tabular}{|c|c|c|c|c|c|c|c|c|}
\hline $\mathrm{DB}$ & WB & $\mathrm{DP}$ & $\mathrm{RH}$ & PV & W & $\mathrm{H}$ & $\mathrm{S}$ & V \\
\hline 80.0 & 80.0 & 80.0 & 100.0 & 1.0323 & .02152 & 42.79 & .0822 & 13.57 \\
\hline 80.0 & 79.0 & 78.7 & 95.7 & .9879 & .02056 & 41.74 & .0802 & 13.55 \\
\hline 80.0 & 78.0 & 77.3 & 91.5 & .9445 & .01963 & 40.72 & .0783 & 13.53 \\
\hline 80.0 & 77.0 & 75.9 & 87.4 & .9020 & .01872 & 39.73 & .0765 & 13.51 \\
\hline 80.0 & 76.0 & 74.5 & 83.3 & .8604 & .01783 & 38.75 & .0746 & 13.49 \\
\hline 80.0 & 75.0 & 73.0 & 79.4 & .8196 & .01696 & 37.80 & .0729 & 13.48 \\
\hline 80.0 & 74.0 & 71.6 & 75.5 & .7797 & .01611 & 36.87 & .0711 & 13.46 \\
\hline 80.0 & 73.0 & 70.1 & 71.7 & .7406 & .01529 & 35.97 & .0694 & 13.44 \\
\hline 80.0 & 72.0 & 68.5 & 68.0 & .7024 & .01448 & 35.08 & .0677 & 13.42 \\
\hline 80.0 & 71.0 & 66.9 & 64.4 & .6649 & .01369 & 34.22 & .0661 & 13.41 \\
\hline 80.0 & 70.0 & 65.3 & 60.9 & .6282 & .01292 & 33.37 & .0645 & 13.39 \\
\hline 80.0 & 69.0 & 63.6 & 57.4 & .5922 & .01216 & 32.54 & .0629 & 13.37 \\
\hline 80.0 & 68.0 & 61.9 & 54.0 & .5570 & .01143 & 31.74 & .0613 & 13.36 \\
\hline 80.0 & 67.0 & 60.1 & 50.6 & .5225 & .01071 & 30.95 & .0598 & 13.34 \\
\hline 80.0 & 66.0 & 58.2 & 47.3 & .4887 & .01000 & 30.18 & .0583 & 13.33 \\
\hline 80.0 & 65.0 & 56.2 & 44.1 & .4556 & .00931 & 29.42 & .0569 & 13.31 \\
\hline 80.0 & 64.0 & 54.2 & 41.0 & .4231 & .00864 & 28.69 & .0555 & 13.30 \\
\hline 80.0 & 63.0 & 52.1 & 37.9 & .3913 & .00798 & 27.97 & .0540 & 13.29 \\
\hline 80.0 & 62.0 & 49.8 & 34.9 & .3602 & .00734 & 27.26 & .0527 & 13.27 \\
\hline 80.0 & 61.0 & 47.5 & 31.9 & .3296 & .00671 & 26.57 & .0513 & 13.26 \\
\hline 80.0 & 60.0 & 44.9 & 29.0 & .2997 & .00609 & 25.90 & .0500 & 13.25 \\
\hline 80.0 & 59.0 & 42.3 & 26.2 & .2703 & .00549 & 25.23 & .0487 & 13.23 \\
\hline 80.0 & 58.0 & 39.4 & 23.4 & .2415 & .00490 & 24.59 & .0474 & 13.22 \\
\hline 80.0 & 57.0 & 36.2 & 20.7 & .2132 & .00432 & 23.96 & .0461 & 13.21 \\
\hline 80.0 & 56.0 & 32.7 & 18.0 & .1856 & .00376 & 23.34 & .0448 & 13.20 \\
\hline 80.0 & 55.0 & 29.2 & 15.3 & .1584 & .00321 & 22.73 & .0436 & 13.19 \\
\hline 80.0 & 54.0 & 25.2 & 12.8 & .1317 & .00266 & 22.14 & .0424 & 13.18 \\
\hline 80.0 & 53.0 & 20.6 & 10.2 & .1056 & .00213 & 21.55 & .0412 & 13.16 \\
\hline 80.0 & 52.0 & 14.8 & 7.7 & .0799 & .00161 & 20.98 & .0400 & 13.15 \\
\hline 80.0 & 51.0 & 7.3 & 5.3 & .0547 & .00110 & 20.43 & .0388 & 13.14 \\
\hline 80.0 & 50.0 & -4.3 & 2.9 & .0300 & .00061 & 19.88 & .0376 & 13.13 \\
\hline 80.0 & 49.0 & -33.3 & .6 & .0058 & .00012 & 19.34 & .0364 & 13.12 \\
\hline
\end{tabular}


$\mathrm{PB}=31.02$, Altitude $=-1000-$ Continued

$\begin{array}{rrrrrrrrr}\text { DB } & \text { WB } & \text { DP } & \text { RH } & \text { PV } & \text { W } & \text { H } & \text { S } & \text { V } \\ & & & & & & & & \\ 120.0 & 120.0 & 120.0 & 100.0 & 3.4476 & .07826 & 115.94 & .2126 & 15.86 \\ 120.0 & 118.0 & 117.8 & 94.0 & 3.2393 & .07298 & 110.06 & .2024 & 15.74 \\ 120.0 & 116.0 & 115.5 & 88.2 & 3.0397 & .06798 & 104.51 & .1927 & 15.63 \\ 120.0 & 114.0 & 113.2 & 82.6 & 2.8483 & .06326 & 99.26 & .1835 & 15.52 \\ 120.0 & 112.0 & 110.9 & 77.3 & 2.6649 & .05880 & 94.29 & .1748 & 15.42 \\ 120.0 & 110.0 & 108.5 & 72.2 & 2.4892 & .05458 & 89.60 & .1665 & 15.32 \\ 120.0 & 108.0 & 106.1 & 67.3 & 2.3208 & .05058 & 85.15 & .1586 & 15.23 \\ 120.0 & 106.0 & 103.7 & 62.6 & 2.1595 & .04680 & 80.94 & .1511 & 15.15 \\ 120.0 & 104.0 & 101.2 & 58.2 & 2.0050 & .04322 & 76.96 & .1440 & 15.07 \\ 120.0 & 102.0 & 98.7 & 53.9 & 1.8571 & .03982 & 73.18 & .1372 & 14.99 \\ 120.0 & 100.0 & 96.1 & 49.8 & 1.7154 & .03660 & 69.59 & .1308 & 14.92 \\ 120.0 & 98.0 & 93.4 & 45.8 & 1.5797 & .03355 & 66.19 & .1246 & 14.85 \\ 120.0 & 96.0 & 90.6 & 42.1 & 1.4497 & .03065 & 62.97 & .1188 & 14.79 \\ 120.0 & 94.0 & 87.8 & 38.4 & 1.3253 & .02790 & 59.91 & .1132 & 14.72 \\ 120.0 & 92.0 & 84.8 & 35.0 & 1.2062 & .02529 & 57.00 & .1078 & 14.66 \\ 120.0 & 90.0 & 81.7 & 31.7 & 1.0921 & .02281 & 54.24 & .1027 & 14.61 \\ 120.0 & 88.0 & 78.5 & 28.5 & .9829 & .02045 & 51.61 & .0979 & 14.56 \\ 120.0 & 86.0 & 75.1 & 25.5 & .8783 & .01821 & 49.12 & .0932 & 14.51 \\ 120.0 & 84.0 & 71.5 & 22.6 & .7782 & .01608 & 46.75 & .0888 & 14.46 \\ 120.0 & 82.0 & 67.7 & 19.8 & .6822 & .01405 & 44.49 & .0845 & 14.41 \\ 120.0 & 80.0 & 63.5 & 17.1 & .5904 & .01212 & 42.34 & .0804 & 14.37 \\ 120.0 & 78.0 & 58.9 & 14.6 & .5023 & .01029 & 40.29 & .0765 & 14.33 \\ 120.0 & 76.0 & 53.9 & 12.1 & .4180 & .00853 & 38.34 & .0727 & 14.29 \\ 120.0 & 74.0 & 48.1 & 9.8 & .3372 & .00687 & 36.48 & .0691 & 14.25 \\ 120.0 & 72.0 & 41.2 & 7.5 & .2597 & .00528 & 34.71 & .0656 & 14.21 \\ 120.0 & 70.0 & 32.7 & 5.4 & .1855 & .00376 & 33.02 & .0622 & 14.18 \\ 120.0 & 68.0 & 22.2 & 3.3 & .1143 & .00231 & 31.41 & .0589 & 14.15 \\ 120.0 & 66.0 & 3.9 & 1.3 & .0460 & .00093 & 29.87 & .0556 & 14.11\end{array}$


$\mathrm{PB}=31.02$, Altitude $=-1000-$ Continued

\begin{tabular}{|c|c|c|c|c|c|c|c|c|}
\hline $\mathrm{DB}$ & WB & $\mathrm{DP}$ & $\mathrm{RH}$ & PV & W & $\mathrm{H}$ & $\mathrm{S}$ & $\mathrm{V}$ \\
\hline 160.0 & 160.0 & 160.0 & 100.0 & 9.6555 & .28371 & 358.97 & .6192 & 21.88 \\
\hline 160.0 & 158.0 & 157.9 & 95.1 & 9.1863 & 26407 & 336.81 & .5829 & 21.41 \\
\hline 160.0 & 156.0 & 155.8 & 90.5 & 8.7346 & .24595 & 316.37 & .5493 & 20.98 \\
\hline 160.0 & 154.0 & 153.7 & 86.0 & 8.2998 & 22919 & 297.46 & .5181 & 20.58 \\
\hline 160.0 & 152.0 & 151.6 & 81.6 & 7.8813 & .21366 & 279.93 & .4892 & 20.21 \\
\hline 160.0 & 150.0 & 149.5 & 77.5 & 7.4787 & .19924 & 263.66 & .4622 & 19.86 \\
\hline 160.0 & 148.0 & 147.4 & 73.4 & 7.0915 & .18584 & 248.52 & .4370 & 19.54 \\
\hline 160.0 & 146.0 & 145.2 & 69.6 & 6.7191 & .17335 & 234.43 & .4135 & 19.24 \\
\hline 160.0 & 144.0 & 143.0 & 65.9 & 6.3611 & .16170 & 221.28 & .3915 & 18.96 \\
\hline 160.0 & 142.0 & 140.9 & 62.3 & 6.0171 & .15083 & 208.99 & .3709 & 18.70 \\
\hline 160.0 & 140.0 & 138.7 & 58.9 & 5.6865 & .14066 & 197.51 & .3515 & 18.46 \\
\hline 160.0 & 138.0 & 136.5 & 55.6 & 5.3688 & .13114 & 186.75 & .3334 & 18.23 \\
\hline 160.0 & 136.0 & 134.2 & 52.4 & 5.0638 & .12221 & 176.67 & .3163 & 18.01 \\
\hline 160.0 & 134.0 & 132.0 & 49.4 & 4.7709 & .11384 & 167.21 & .3002 & 17.81 \\
\hline 160.0 & 132.0 & 129.7 & 46.5 & 4.4897 & .10598 & 158.33 & .2850 & 17.62 \\
\hline 160.0 & 130.0 & 127.4 & 43.7 & 4.2198 & .09859 & 149.98 & .2707 & 17.45 \\
\hline 160.0 & 128.0 & 125.0 & 41.0 & 3.9608 & .09165 & 142.13 & .2572 & 17.28 \\
\hline 160.0 & 126.0 & 122.7 & 38.4 & 3.7124 & .08510 & 134.73 & .2445 & 17.12 \\
\hline 160.0 & 124.0 & 120.3 & 36.0 & 3.4741 & .07894 & 127.77 & .2324 & 16.97 \\
\hline 160.0 & 122.0 & 117.8 & 33.6 & 3.2455 & .07313 & 121.20 & .2210 & 16.83 \\
\hline 160.0 & 120.0 & 115.4 & 31.3 & 3.0264 & .06765 & 115.00 & .2102 & 16.70 \\
\hline 160.0 & 118.0 & 112.8 & 29.2 & 2.8163 & .06248 & 109.16 & .1999 & 16.58 \\
\hline 160.0 & 116.0 & 110.2 & 27.1 & 2.6150 & .05759 & 103.63 & .1902 & 16.46 \\
\hline 160.0 & 114.0 & 107.6 & 25.1 & 2.4220 & .05298 & 98.41 & .1810 & 16.35 \\
\hline 160.0 & 112.0 & 104.9 & 23.2 & 2.2372 & .04862 & 93.48 & .1723 & 16.24 \\
\hline 160.0 & 110.0 & 102.1 & 21.3 & 2.0601 & .04449 & 88.81 & .1639 & 16.14 \\
\hline 160.0 & 108.0 & 99.3 & 19.6 & 1.8904 & .04058 & 84.39 & .1560 & 16.05 \\
\hline 160.0 & 106.0 & 96.3 & 17.9 & 1.7279 & .03689 & 80.21 & .1485 & 15.96 \\
\hline 160.0 & 104.0 & 93.2 & 16.3 & 1.5724 & .03338 & 76.25 & .1414 & 15.87 \\
\hline 160.0 & 102.0 & 90.0 & 14.7 & 1.4234 & .03007 & 72.49 & .1345 & 15.79 \\
\hline 160.0 & 100.0 & 86.7 & 13.3 & 1.2807 & .02692 & 68.93 & .1280 & 15.72 \\
\hline 160.0 & 98.0 & 83.2 & 11.9 & 1.1442 & .02394 & 65.56 & .1218 & 15.65 \\
\hline 160.0 & 96.0 & 79.4 & 10.5 & 1.0135 & .02111 & 62.36 & .1159 & 15.58 \\
\hline 160.0 & 94.0 & 75.5 & 9.2 & .8883 & .01842 & 59.32 & .1103 & 15.51 \\
\hline 160.0 & 92.0 & 71.1 & 8.0 & .7685 & .01588 & 56.44 & .1049 & 15.45 \\
\hline 160.0 & 90.0 & 66.4 & 6.8 & .6539 & .01346 & 53.70 & .0997 & 15.39 \\
\hline 160.0 & 88.0 & 61.2 & 5.6 & .5441 & 01116 & 51.09 & .0947 & 15.34 \\
\hline 160.0 & 86.0 & 55.2 & 4.5 & .4391 & .00897 & 48.62 & .0900 & 15.29 \\
\hline 160.0 & 84.0 & 48.2 & 3.5 & .3385 & .00689 & 46.27 & .0854 & 15.23 \\
\hline 160.0 & 82.0 & 39.4 & 2.5 & .2422 & .00492 & 44.03 & .0810 & 15.19 \\
\hline 160.0 & 80.0 & 28.0 & 1.6 & .1500 & .00304 & 41.90 & .0767 & 15.14 \\
\hline 160.0 & 78.0 & 9.6 & .6 & .0617 & .00125 & 39.88 & .0725 & 15.10 \\
\hline
\end{tabular}


PB $=29.92$, Altitude $=0$

\begin{tabular}{|c|c|c|c|c|c|c|c|c|}
\hline $\mathrm{DB}$ & WB & $\mathrm{DP}$ & $\mathrm{RH}$ & PV & W & $\mathrm{H}$ & S & V \\
\hline-40.0 & -40.0 & -40.0 & 100.0 & .0038 & .00008 & -9.53 & -.0217 & 10.57 \\
\hline $\begin{array}{l}.0 \\
.0 \\
.0 \\
.0 \\
.0\end{array}$ & $\begin{array}{r}.0 \\
-.1 \\
-.2 \\
-.3 \\
-.4\end{array}$ & $\begin{array}{r}.0 \\
-.6 \\
-1.2 \\
-1.8 \\
-2.4\end{array}$ & $\begin{array}{r}100.0 \\
97.0 \\
93.9 \\
90.9 \\
87.9\end{array}$ & $\begin{array}{l}.0376 \\
.0365 \\
.0354 \\
.0342 \\
.0331\end{array}$ & $\begin{array}{l}.00079 \\
.00076 \\
.00074 \\
.00072 \\
.00069\end{array}$ & $\begin{array}{l}.83 \\
.81 \\
.78 \\
.76 \\
.73\end{array}$ & $\begin{array}{l}.0019 \\
.0019 \\
.0018 \\
.0018 \\
.0017\end{array}$ & $\begin{array}{l}11.59 \\
11.59 \\
11.59 \\
11.59 \\
11.59\end{array}$ \\
\hline $\begin{array}{l}.0 \\
.0 \\
.0 \\
.0 \\
.0\end{array}$ & $\begin{array}{l}-.5 \\
-.6 \\
-.7 \\
-.8 \\
-.9\end{array}$ & $\begin{array}{l}-3.1 \\
-3.8 \\
-4.5 \\
-5.2 \\
-6.0\end{array}$ & $\begin{array}{l}84.9 \\
81.9 \\
78.9 \\
75.9 \\
72.9\end{array}$ & $\begin{array}{l}.0320 \\
.0308 \\
.0297 \\
.0286 \\
.0274\end{array}$ & $\begin{array}{l}.00067 \\
.00064 \\
.00062 \\
.00060 \\
.00057\end{array}$ & $\begin{array}{l}.71 \\
.68 \\
.66 \\
.63 \\
.61\end{array}$ & $\begin{array}{l}.0016 \\
.0016 \\
.0015 \\
.0015 \\
.0014\end{array}$ & $\begin{array}{l}11.59 \\
11.59 \\
11.59 \\
11.59 \\
11.59\end{array}$ \\
\hline $\begin{array}{l}.0 \\
.0 \\
.0 \\
.0 \\
.0\end{array}$ & $\begin{array}{l}-1.0 \\
-1.1 \\
-1.2 \\
-1.3 \\
-1.4\end{array}$ & $\begin{array}{r}-6.7 \\
-7.6 \\
-8.4 \\
-9.3 \\
-10.2\end{array}$ & $\begin{array}{l}69.8 \\
66.8 \\
63.8 \\
60.8 \\
57.9\end{array}$ & $\begin{array}{l}.0263 \\
.0252 \\
.0240 \\
.0229 \\
.0218\end{array}$ & $\begin{array}{l}.00055 \\
.00053 \\
.00050 \\
.00048 \\
.00046\end{array}$ & $\begin{array}{l}.58 \\
.56 \\
.53 \\
.51 \\
.48\end{array}$ & $\begin{array}{l}.0014 \\
.0013 \\
.0013 \\
.0012 \\
.0011\end{array}$ & $\begin{array}{l}11.59 \\
11.59 \\
11.59 \\
11.59 \\
11.59\end{array}$ \\
\hline $\begin{array}{l}.0 \\
.0 \\
.0 \\
.0 \\
.0\end{array}$ & $\begin{array}{l}-1.5 \\
-1.6 \\
-1.7 \\
-1.8 \\
-1.9\end{array}$ & $\begin{array}{l}-11.2 \\
-12.2 \\
-13.3 \\
-14.4 \\
-15.6\end{array}$ & $\begin{array}{l}54.9 \\
51.9 \\
48.9 \\
45.9 \\
42.9\end{array}$ & $\begin{array}{l}.0207 \\
.0195 \\
.0184 \\
.0173 \\
.0162\end{array}$ & $\begin{array}{l}.00043 \\
.00041 \\
.00038 \\
.00036 \\
.00034\end{array}$ & $\begin{array}{l}.46 \\
.43 \\
.41 \\
.38 \\
.36\end{array}$ & $\begin{array}{l}.0011 \\
.0010 \\
.0010 \\
.0009 \\
.0009\end{array}$ & $\begin{array}{l}11.59 \\
11.59 \\
11.59 \\
11.59 \\
11.59\end{array}$ \\
\hline $\begin{array}{l}.0 \\
.0 \\
.0 \\
.0 \\
.0 \\
.0\end{array}$ & $\begin{array}{l}-2.0 \\
-2.1 \\
-2.2 \\
-2.3 \\
-2.4 \\
-2.5\end{array}$ & $\begin{array}{l}-16.9 \\
-18.2 \\
-19.7 \\
-21.3 \\
-23.0 \\
-25.0\end{array}$ & $\begin{array}{l}39.9 \\
37.0 \\
34.0 \\
31.0 \\
28.1 \\
25.1\end{array}$ & $\begin{array}{l}.0150 \\
.0139 \\
.0128 \\
.0117 \\
.0106 \\
.0094\end{array}$ & $\begin{array}{l}.00031 \\
.00029 \\
.00027 \\
.00024 \\
.00022 \\
.00020\end{array}$ & $\begin{array}{l}.33 \\
.31 \\
.28 \\
.26 \\
.23 \\
.21\end{array}$ & $\begin{array}{l}.0008 \\
.0007 \\
.0007 \\
.0006 \\
.0006 \\
.0005\end{array}$ & $\begin{array}{l}11.58 \\
11.58 \\
11.58 \\
11.58 \\
11.58 \\
11.58\end{array}$ \\
\hline $\begin{array}{l}40.0 \\
40.0 \\
40.0 \\
40.0 \\
40.0\end{array}$ & $\begin{array}{l}40.0 \\
39.0 \\
38.0 \\
37.0 \\
36.0\end{array}$ & $\begin{array}{l}40.0 \\
37.8 \\
35.5 \\
33.1 \\
30.6\end{array}$ & $\begin{array}{r}100.0 \\
91.9 \\
83.9 \\
76.0 \\
68.2\end{array}$ & $\begin{array}{l}.2477 \\
.2275 \\
.2077 \\
.1882 \\
.1690\end{array}$ & $\begin{array}{l}.00521 \\
.00479 \\
.00437 \\
.00395 \\
.00355\end{array}$ & $\begin{array}{l}15.23 \\
14.77 \\
14.32 \\
13.87 \\
13.43\end{array}$ & $\begin{array}{l}.0319 \\
.0309 \\
.0300 \\
.0291 \\
.0282\end{array}$ & $\begin{array}{l}12.70 \\
12.69 \\
12.68 \\
12.67 \\
12.66\end{array}$ \\
\hline $\begin{array}{l}40.0 \\
40.0 \\
40.0 \\
40.0 \\
40.0\end{array}$ & $\begin{array}{l}35.0 \\
34.0 \\
33.0 \\
32.0 \\
31.0\end{array}$ & $\begin{array}{l}28.0 \\
25.2 \\
22.0 \\
20.5 \\
16.7\end{array}$ & $\begin{array}{l}60.6 \\
53.1 \\
45.7 \\
42.5 \\
35.4\end{array}$ & $\begin{array}{l}.1501 \\
.1315 \\
.1132 \\
.1052 \\
.0877\end{array}$ & $\begin{array}{l}.00315 \\
.00276 \\
.00237 \\
.00220 \\
.00184\end{array}$ & $\begin{array}{l}13.00 \\
12.58 \\
12.17 \\
11.98 \\
11.59\end{array}$ & $\begin{array}{l}.0274 \\
.0265 \\
.0256 \\
.0253 \\
.0244\end{array}$ & $\begin{array}{l}12.65 \\
12.65 \\
12.64 \\
12.63 \\
12.63\end{array}$ \\
\hline $\begin{array}{l}40.0 \\
40.0 \\
40.0 \\
40.0 \\
40.0\end{array}$ & $\begin{array}{l}30.0 \\
29.0 \\
28.0 \\
27.0 \\
26.0\end{array}$ & $\begin{array}{r}12.3 \\
6.9 \\
-.2 \\
-10.9 \\
-35.6\end{array}$ & $\begin{array}{r}28.5 \\
21.7 \\
15.0 \\
8.5 \\
2.0\end{array}$ & $\begin{array}{l}.0705 \\
.0537 \\
.0372 \\
.0209 \\
.0050\end{array}$ & $\begin{array}{l}.00148 \\
.00112 \\
.00078 \\
.00044 \\
.00010\end{array}$ & $\begin{array}{r}11.20 \\
10.82 \\
10.45 \\
10.08 \\
9.72\end{array}$ & $\begin{array}{l}.0236 \\
.0228 \\
.0220 \\
.0212 \\
.0203\end{array}$ & $\begin{array}{l}12.62 \\
12.61 \\
12.61 \\
12.60 \\
12.59\end{array}$ \\
\hline
\end{tabular}


PB $=29.92$, Altitude $=0-$ Continued

\begin{tabular}{|c|c|c|c|c|c|c|c|c|}
\hline $\mathrm{DB}$ & WB & $\mathrm{DP}$ & $\mathrm{RH}$ & PV & W & $\mathrm{H}$ & $\mathrm{S}$ & V \\
\hline 80.0 & 80.0 & 80.0 & 100.0 & 1.0323 & .02233 & 43.69 & .0864 & 14.09 \\
\hline 80.0 & 79.0 & 78.7 & 95.7 & .9883 & .02135 & 42.61 & .0844 & 14.07 \\
\hline 80.0 & 78.0 & 77.3 & 91.6 & .9453 & .02039 & 41.56 & .0824 & 14.05 \\
\hline 80.0 & 77.0 & 75.9 & 87.5 & .9032 & .01945 & 40.53 & .0805 & 14.03 \\
\hline 80.0 & 76.0 & 74.5 & 83.5 & .8620 & .01854 & 39.53 & .0786 & 14.01 \\
\hline 80.0 & 75.0 & 73.1 & 79.6 & .8216 & .01764 & 38.55 & .0768 & 13.99 \\
\hline 80.0 & 74.0 & 71.7 & 75.8 & .7821 & .01677 & 37.60 & .0750 & 13.97 \\
\hline 80.0 & 73.0 & 70.2 & 72.0 & .7435 & .01592 & 36.67 & .0733 & 13.95 \\
\hline 80.0 & 72.0 & 68.6 & 68.4 & .7056 & .01509 & 35.76 & .0715 & 13.93 \\
\hline 80.0 & 71.0 & 67.1 & 64.8 & .6685 & .01428 & 34.87 & .0698 & 13.91 \\
\hline 80.0 & 70.0 & 65.5 & 61.2 & .6322 & .01349 & 34.00 & .0682 & 13.90 \\
\hline 80.0 & 69.0 & 63.8 & 57.8 & .5967 & .01271 & 33.15 & .0666 & 13.88 \\
\hline 80.0 & 68.0 & 62.1 & 54.4 & .5618 & .01196 & 32.32 & .0650 & 13.86 \\
\hline 80.0 & 67.0 & 60.3 & 51.1 & .5277 & .01122 & 31.51 & .0634 & 13.85 \\
\hline 80.0 & 66.0 & 58.5 & 47.9 & .4943 & .01050 & 30.72 & .0619 & 13.83 \\
\hline 80.0 & 65.0 & 56.6 & 44.7 & .4616 & .00979 & 29.95 & .0604 & 13.81 \\
\hline 80.0 & 64.0 & 54.6 & 41.6 & .4295 & .00910 & 29.19 & .0589 & 13.80 \\
\hline 80.0 & 63.0 & 52.5 & 38.6 & .3981 & .00842 & 28.45 & .0575 & 13.79 \\
\hline 80.0 & 62.0 & 50.4 & 35.6 & .3674 & .00777 & 27.73 & .0561 & 13.77 \\
\hline 80.0 & 61.0 & 48.1 & 32.7 & .3372 & .00712 & 27.02 & .0547 & 13.76 \\
\hline 80.0 & 60.0 & 45.6 & 29.8 & .3076 & .00649 & 26.33 & .0533 & 13.74 \\
\hline 80.0 & 59.0 & 43.0 & 27.0 & .2787 & .00587 & 25.66 & .0520 & 13.73 \\
\hline 80.0 & 58.0 & 40.3 & 24.2 & .2502 & .00527 & 24.99 & .0507 & 13.72 \\
\hline 80.0 & 57.0 & 37.3 & 21.5 & .2224 & .00468 & 24.35 & .0494 & 13.70 \\
\hline 80.0 & 56.0 & 34.0 & 18.9 & .1951 & .00410 & 23.71 & .0481 & 13.69 \\
\hline 80.0 & 55.0 & 30.5 & 16.3 & .1683 & .00353 & 23.09 & .0468 & 13.68 \\
\hline 80.0 & 54.0 & 26.8 & 13.8 & .1420 & .00298 & 22.49 & .0456 & 13.67 \\
\hline 80.0 & 53.0 & 22.6 & 11.3 & .1163 & .00244 & 21.89 & .0444 & 13.65 \\
\hline 80.0 & 52.0 & 17.5 & 8.8 & .0910 & .00191 & 21.31 & .0432 & 13.64 \\
\hline 80.0 & 51.0 & 11.0 & 6.4 & .0662 & .00139 & 20.74 & .0419 & 13.63 \\
\hline 80.0 & 50.0 & 2.1 & 4.1 & .0419 & .00088 & 20.18 & .0407 & 13.62 \\
\hline 80.0 & 49.0 & -13.6 & 1.7 & .0180 & .00038 & 19.63 & .0395 & 13.61 \\
\hline
\end{tabular}


PB $=29.92$, Altitude $=0-$ Continued

$\begin{array}{rrrrrrrrr}\text { DB } & \text { WB } & \text { DP } & \text { RH } & \text { PV } & \text { W } & \text { H } & \text { S } & \text { V } \\ 120.0 & 120.0 & 120.0 & 100.0 & 3.4476 & .08151 & 119.55 & .2217 & 16.52 \\ 120.0 & 118.0 & 117.8 & 94.0 & 3.2402 & .07600 & 113.43 & .2110 & 16.39 \\ 120.0 & 116.0 & 115.5 & 88.2 & 3.0413 & .07080 & 107.64 & .2009 & 16.27 \\ 120.0 & 114.0 & 113.2 & 82.7 & 2.8508 & .06589 & 102.18 & .1914 & 16.15 \\ 120.0 & 112.0 & 110.9 & 77.4 & 2.6682 & .06125 & 97.02 & .1823 & 16.04 \\ 120.0 & 110.0 & 108.6 & 72.3 & 2.4933 & .05686 & 92.14 & .1737 & 15.94 \\ 120.0 & 108.0 & 106.2 & 67.5 & 2.3257 & .05271 & 87.53 & .1655 & 15.85 \\ 120.0 & 106.0 & 103.8 & 62.8 & 2.1652 & .04879 & 83.16 & .1577 & 15.75 \\ 120.0 & 104.0 & 101.3 & 58.3 & 2.0116 & .04507 & 79.02 & .1504 & 15.67 \\ 120.0 & 102.0 & 98.8 & 54.1 & 1.8644 & .04155 & 75.11 & .1433 & 15.58 \\ 120.0 & 100.0 & 96.2 & 50.0 & 1.7235 & .03822 & 71.39 & .1366 & 15.51 \\ 120.0 & 98.0 & 93.6 & 46.1 & 1.5886 & .03505 & 67.88 & .1303 & 15.43 \\ 120.0 & 96.0 & 90.8 & 42.3 & 1.4595 & .03206 & 64.54 & .1242 & 15.36 \\ 120.0 & 94.0 & 88.0 & 38.7 & 1.3359 & .02921 & 61.37 & .1184 & 15.30 \\ 120.0 & 92.0 & 85.1 & 35.3 & 1.2175 & .02651 & 58.37 & .1129 & 15.23 \\ 120.0 & 90.0 & 82.1 & 32.0 & 1.1043 & .02395 & 55.51 & .1077 & 15.17 \\ 120.0 & 88.0 & 78.9 & 28.9 & .9958 & .02152 & 52.80 & .1026 & 15.12 \\ 120.0 & 86.0 & 75.6 & 25.9 & .8920 & .01920 & 50.23 & .0978 & 15.06 \\ 120.0 & 84.0 & 72.1 & 23.0 & .7927 & .01700 & 47.78 & .0932 & 15.01 \\ 120.0 & 82.0 & 68.3 & 20.2 & .6975 & .01491 & 45.45 & .0888 & 14.96 \\ 120.0 & 80.0 & 64.3 & 17.6 & .6065 & .01293 & 43.24 & .0846 & 14.91 \\ 120.0 & 78.0 & 59.9 & 15.1 & .5192 & .01103 & 41.13 & .0806 & 14.87 \\ 120.0 & 76.0 & 55.0 & 12.6 & .4357 & .00923 & 39.12 & .0767 & 14.83 \\ 120.0 & 74.0 & 49.5 & 10.3 & .3557 & .00752 & 37.21 & .0730 & 14.79 \\ 120.0 & 72.0 & 43.1 & 8.1 & .2790 & .00588 & 35.39 & .0694 & 14.75 \\ 120.0 & 70.0 & 35.3 & 6.0 & .2055 & .00432 & 33.65 & .0659 & 14.71 \\ 120.0 & 68.0 & 25.8 & 3.9 & .1351 & .00283 & 31.99 & .0625 & 14.68 \\ 120.0 & 66.0 & 11.4 & 2.0 & .0675 & .00141 & 30.41 & .0592 & 14.65 \\ 120.0 & 64.0 & -44.9 & .1 & .0028 & .00006 & 28.90 & .0559 & 14.61\end{array}$


PB $=29.92$, Altitude $=0$ - Continued

\begin{tabular}{|c|c|c|c|c|c|c|c|c|}
\hline DB & WB & $\mathrm{DP}$ & $\mathrm{RH}$ & PV & W & $\mathrm{H}$ & $\mathrm{S}$ & $\mathrm{V}$ \\
\hline $\begin{array}{l}160.0 \\
160.0 \\
160.0 \\
160.0 \\
160.0\end{array}$ & $\begin{array}{l}160.0 \\
158.0 \\
156.0 \\
154.0 \\
152.0\end{array}$ & $\begin{array}{l}160.0 \\
157.9 \\
155.8 \\
153.7 \\
151.6\end{array}$ & $\begin{array}{r}100.0 \\
95.1 \\
90.5 \\
86.0 \\
81.7\end{array}$ & $\begin{array}{l}9.6555 \\
9.1872 \\
8.7363 \\
8.3023 \\
7.8847\end{array}$ & $\begin{array}{l}.29909 \\
.27810 \\
.25877 \\
.24093 \\
.22443\end{array}$ & $\begin{array}{l}376.34 \\
352.66 \\
330.86 \\
310.73 \\
292.11\end{array}$ & $\begin{array}{l}.6512 \\
.6124 \\
.5766 \\
.5434 \\
.5127\end{array}$ & $\begin{array}{l}23.07 \\
22.55 \\
22.07 \\
21.63 \\
21.22\end{array}$ \\
\hline $\begin{array}{l}160.0 \\
160.0 \\
160.0 \\
160.0 \\
160.0\end{array}$ & $\begin{array}{l}150.0 \\
148.0 \\
146.0 \\
144.0 \\
142.0\end{array}$ & $\begin{array}{l}149.5 \\
147.4 \\
145.2 \\
143.1 \\
140.9\end{array}$ & $\begin{array}{l}77.5 \\
73.5 \\
69.6 \\
66.0 \\
62.4\end{array}$ & $\begin{array}{l}7.4829 \\
7.0965 \\
6.7250 \\
6.3679 \\
6.0246\end{array}$ & $\begin{array}{l}.20915 \\
.19495 \\
.18176 \\
.16947 \\
.15800\end{array}$ & $\begin{array}{l}274.86 \\
258.84 \\
243.94 \\
230.06 \\
217.11\end{array}$ & $\begin{array}{l}.4841 \\
.4574 \\
.4326 \\
.4094 \\
.3876\end{array}$ & $\begin{array}{l}20.84 \\
20.49 \\
20.16 \\
19.85 \\
19.57\end{array}$ \\
\hline $\begin{array}{l}160.0 \\
160.0 \\
160.0 \\
160.0 \\
160.0\end{array}$ & $\begin{array}{l}140.0 \\
138.0 \\
136.0 \\
134.0 \\
132.0\end{array}$ & $\begin{array}{l}138.7 \\
136.5 \\
134.3 \\
132.1 \\
129.8\end{array}$ & $\begin{array}{l}59.0 \\
55.7 \\
52.5 \\
49.5 \\
46.6\end{array}$ & $\begin{array}{l}5.6948 \\
5.3780 \\
5.0738 \\
4.7817 \\
4.5013\end{array}$ & $\begin{array}{l}.14730 \\
.13728 \\
.12791 \\
.11913 \\
.11089\end{array}$ & $\begin{array}{l}205.02 \\
193.71 \\
183.12 \\
173.20 \\
163.89\end{array}$ & $\begin{array}{l}.3673 \\
.3481 \\
.3302 \\
.3133 \\
.2974\end{array}$ & $\begin{array}{l}19.30 \\
19.05 \\
18.82 \\
18.60 \\
18.39\end{array}$ \\
\hline $\begin{array}{l}160.0 \\
160.0 \\
160.0 \\
160.0 \\
160.0\end{array}$ & $\begin{array}{l}130.0 \\
128.0 \\
126.0 \\
124.0 \\
122.0\end{array}$ & $\begin{array}{l}127.5 \\
125.2 \\
122.8 \\
120.4 \\
118.0\end{array}$ & $\begin{array}{l}43.8 \\
41.2 \\
38.6 \\
36.1 \\
33.8\end{array}$ & $\begin{array}{l}4.2323 \\
3.9741 \\
3.7264 \\
3.4889 \\
3.2612\end{array}$ & $\begin{array}{l}.10316 \\
.09589 \\
.08905 \\
.08261 \\
.07655\end{array}$ & $\begin{array}{l}155.15 \\
146.93 \\
139.20 \\
131.93 \\
125.07\end{array}$ & $\begin{array}{l}.2825 \\
.2683 \\
.2550 \\
.2424 \\
.2305\end{array}$ & $\begin{array}{l}18.20 \\
18.02 \\
17.85 \\
17.69 \\
17.54\end{array}$ \\
\hline $\begin{array}{l}160.0 \\
160.0 \\
160.0 \\
160.0 \\
160.0\end{array}$ & $\begin{array}{l}120.0 \\
118.0 \\
116.0 \\
114.0 \\
112.0\end{array}$ & $\begin{array}{l}115.5 \\
113.0 \\
110.5 \\
107.9 \\
105.2\end{array}$ & $\begin{array}{l}31.5 \\
29.3 \\
27.3 \\
25.3 \\
23.4\end{array}$ & $\begin{array}{l}3.0429 \\
2.8336 \\
2.6330 \\
2.4409 \\
2.2568\end{array}$ & $\begin{array}{l}.07084 \\
.06545 \\
.06036 \\
.05556 \\
.05102\end{array}$ & $\begin{array}{r}118.61 \\
112.52 \\
106.77 \\
101.34 \\
96.20\end{array}$ & $\begin{array}{l}.2192 \\
.2086 \\
.1985 \\
.1889 \\
.1798\end{array}$ & $\begin{array}{l}17.39 \\
17.26 \\
17.13 \\
17.01 \\
16.90\end{array}$ \\
\hline $\begin{array}{l}160.0 \\
160.0 \\
160.0 \\
160.0 \\
160.0\end{array}$ & $\begin{array}{l}110.0 \\
108.0 \\
106.0 \\
104.0 \\
102.0\end{array}$ & $\begin{array}{r}102.4 \\
99.6 \\
96.7 \\
93.7 \\
90.6\end{array}$ & $\begin{array}{l}21.5 \\
19.8 \\
18.1 \\
16.5 \\
15.0\end{array}$ & $\begin{array}{l}2.0805 \\
1.9117 \\
1.7500 \\
1.5952 \\
1.4470\end{array}$ & $\begin{array}{l}.04673 \\
.04268 \\
.03884 \\
.03521 \\
.03177\end{array}$ & $\begin{array}{l}91.35 \\
86.76 \\
82.42 \\
78.31 \\
74.42\end{array}$ & $\begin{array}{l}.1711 \\
.1629 \\
.1551 \\
.1477 \\
.1406\end{array}$ & $\begin{array}{l}16.79 \\
16.69 \\
16.59 \\
16.50 \\
16.42\end{array}$ \\
\hline $\begin{array}{l}160.0 \\
160.0 \\
160.0 \\
160.0 \\
160.0\end{array}$ & $\begin{array}{r}100.0 \\
98.0 \\
96.0 \\
94.0 \\
92.0\end{array}$ & $\begin{array}{l}87.3 \\
83.8 \\
80.2 \\
76.3 \\
72.2\end{array}$ & $\begin{array}{r}13.5 \\
12.1 \\
10.8 \\
9.5 \\
8.2\end{array}$ & $\begin{array}{r}1.3051 \\
1.1694 \\
1.0394 \\
.9151 \\
.7961\end{array}$ & $\begin{array}{l}.02851 \\
.02542 \\
.02249 \\
.01971 \\
.01708\end{array}$ & $\begin{array}{l}70.73 \\
67.24 \\
63.93 \\
60.78 \\
57.80\end{array}$ & $\begin{array}{l}.1339 \\
.1275 \\
.1214 \\
.1156 \\
.1100\end{array}$ & $\begin{array}{l}16.34 \\
16.26 \\
16.19 \\
16.12 \\
16.05\end{array}$ \\
\hline $\begin{array}{l}160.0 \\
160.0 \\
160.0 \\
160.0 \\
160.0\end{array}$ & $\begin{array}{l}90.0 \\
88.0 \\
86.0 \\
84.0 \\
82.0\end{array}$ & $\begin{array}{l}67.7 \\
62.7 \\
57.0 \\
50.5 \\
42.6\end{array}$ & $\begin{array}{l}7.1 \\
5.9 \\
4.9 \\
3.8 \\
2.8\end{array}$ & $\begin{array}{l}.6822 \\
.5732 \\
.4689 \\
.3691 \\
.2736\end{array}$ & $\begin{array}{l}.01458 \\
.01220 \\
.00995 \\
.00780 \\
.00576\end{array}$ & $\begin{array}{l}54.97 \\
52.28 \\
49.73 \\
47.30 \\
44.99\end{array}$ & $\begin{array}{l}.1046 \\
.0995 \\
.0946 \\
.0899 \\
.0854\end{array}$ & $\begin{array}{l}15.99 \\
15.93 \\
15.87 \\
15.82 \\
15.77\end{array}$ \\
\hline $\begin{array}{l}160.0 \\
160.0 \\
160.0\end{array}$ & $\begin{array}{l}80.0 \\
78.0 \\
76.0\end{array}$ & $\begin{array}{r}32.2 \\
18.3 \\
-22.5\end{array}$ & $\begin{array}{r}1.9 \\
1.0 \\
.1\end{array}$ & $\begin{array}{l}.1822 \\
.0946 \\
.0109\end{array}$ & $\begin{array}{l}.00383 \\
.00198 \\
.00023\end{array}$ & $\begin{array}{l}42.80 \\
40.71 \\
38.72\end{array}$ & $\begin{array}{l}.0810 \\
.0767 \\
.0724\end{array}$ & $\begin{array}{l}15.72 \\
15.67 \\
15.63\end{array}$ \\
\hline
\end{tabular}


$\mathrm{PB}=28.86$, Altitude $=1000$

\begin{tabular}{|c|c|c|c|c|c|c|c|c|}
\hline $\mathrm{DB}$ & WB & $\mathrm{DP}$ & $\mathrm{RH}$ & PV & W & $\mathrm{H}$ & $\mathrm{S}$ & $\mathrm{V}$ \\
\hline-40.0 & -40.0 & -40.0 & 100.0 & .0038 & .00008 & -9.52 & -.0192 & 10.96 \\
\hline $\begin{array}{l}.0 \\
.0 \\
.0 \\
.0 \\
.0\end{array}$ & $\begin{array}{r}.0 \\
-.1 \\
-.2 \\
-.3 \\
-.4\end{array}$ & $\begin{array}{r}.0 \\
-.6 \\
-1.2 \\
-1.8 \\
-2.4\end{array}$ & $\begin{array}{r}100.0 \\
97.1 \\
94.1 \\
91.2 \\
88.3\end{array}$ & $\begin{array}{l}.0376 \\
.0365 \\
.0354 \\
.0343 \\
.0332\end{array}$ & $\begin{array}{l}.00082 \\
.00079 \\
.00077 \\
.00074 \\
.00072\end{array}$ & $\begin{array}{l}.87 \\
.84 \\
.82 \\
.79 \\
.77\end{array}$ & $\begin{array}{l}.0045 \\
.0044 \\
.0044 \\
.0043 \\
.0043\end{array}$ & $\begin{array}{l}12.02 \\
12.02 \\
12.02 \\
12.02 \\
12.02\end{array}$ \\
\hline $\begin{array}{l}.0 \\
.0 \\
.0 \\
.0 \\
.0\end{array}$ & $\begin{array}{l}-.5 \\
-.6 \\
-.7 \\
-.8 \\
-.9\end{array}$ & $\begin{array}{l}-3.0 \\
-3.7 \\
-4.3 \\
-5.0 \\
-5.8\end{array}$ & $\begin{array}{l}85.3 \\
82.4 \\
79.5 \\
76.6 \\
73.6\end{array}$ & $\begin{array}{l}.0321 \\
.0310 \\
.0299 \\
.0288 \\
.0277\end{array}$ & $\begin{array}{l}.00070 \\
.00067 \\
.00065 \\
.00062 \\
.00060\end{array}$ & $\begin{array}{l}.74 \\
.72 \\
.69 \\
.67 \\
.64\end{array}$ & $\begin{array}{l}.0042 \\
.0041 \\
.0041 \\
.0040 \\
.0040\end{array}$ & $\begin{array}{l}12.02 \\
12.02 \\
12.02 \\
12.02 \\
12.02\end{array}$ \\
\hline $\begin{array}{l}.0 \\
.0 \\
.0 \\
.0 \\
.0\end{array}$ & $\begin{array}{l}-1.0 \\
-1.1 \\
-1.2 \\
-1.3 \\
-1.4\end{array}$ & $\begin{array}{l}-6.5 \\
-7.3 \\
-8.1 \\
-8.9 \\
-9.8\end{array}$ & $\begin{array}{l}70.7 \\
67.8 \\
64.9 \\
62.0 \\
59.1\end{array}$ & $\begin{array}{l}.0266 \\
.0255 \\
.0244 \\
.0233 \\
.0222\end{array}$ & $\begin{array}{l}.00058 \\
.00055 \\
.00053 \\
.00051 \\
.00048\end{array}$ & $\begin{array}{l}.62 \\
.59 \\
.56 \\
.54 \\
.51\end{array}$ & $\begin{array}{l}.0039 \\
.0039 \\
.0038 \\
.0037 \\
.0037\end{array}$ & $\begin{array}{l}12.02 \\
12.02 \\
12.01 \\
12.01 \\
12.01\end{array}$ \\
\hline $\begin{array}{l}.0 \\
.0 \\
.0 \\
.0 \\
.0\end{array}$ & $\begin{array}{l}-1.5 \\
-1.6 \\
-1.7 \\
-1.8 \\
-1.9\end{array}$ & $\begin{array}{l}-10.7 \\
-11.7 \\
-12.7 \\
-13.8 \\
-14.9\end{array}$ & $\begin{array}{l}56.2 \\
53.3 \\
50.4 \\
47.5 \\
44.6\end{array}$ & $\begin{array}{l}.0212 \\
.0201 \\
.0190 \\
.0179 \\
.0168\end{array}$ & $\begin{array}{l}.00046 \\
.00043 \\
.00041 \\
.00039 \\
.00036\end{array}$ & $\begin{array}{l}.49 \\
.46 \\
.44 \\
.41 \\
.39\end{array}$ & $\begin{array}{l}.0036 \\
.0036 \\
.0035 \\
.0035 \\
.0034\end{array}$ & $\begin{array}{l}12.01 \\
12.01 \\
12.01 \\
12.01 \\
12.01\end{array}$ \\
\hline $\begin{array}{l}.0 \\
.0 \\
.0 \\
.0 \\
.0\end{array}$ & $\begin{array}{l}-2.0 \\
-2.1 \\
-2.2 \\
-2.3 \\
-2.4\end{array}$ & $\begin{array}{l}-16.1 \\
-17.4 \\
-18.7 \\
-20.2 \\
-21.8\end{array}$ & $\begin{array}{l}41.7 \\
38.8 \\
35.9 \\
33.1 \\
30.2\end{array}$ & $\begin{array}{l}.0157 \\
.0146 \\
.0135 \\
.0124 \\
.0114\end{array}$ & $\begin{array}{l}.00034 \\
.00032 \\
.00029 \\
.00027 \\
.00025\end{array}$ & $\begin{array}{l}.36 \\
.34 \\
.31 \\
.29 \\
.26\end{array}$ & $\begin{array}{l}.0033 \\
.0033 \\
.0032 \\
.0032 \\
.0031\end{array}$ & $\begin{array}{l}12.01 \\
12.01 \\
12.01 \\
12.01 \\
12.01\end{array}$ \\
\hline $\begin{array}{l}.0 \\
.0\end{array}$ & $\begin{array}{l}-2.5 \\
-2.6\end{array}$ & $\begin{array}{l}-23.5 \\
-25.4\end{array}$ & $\begin{array}{l}27.3 \\
24.4\end{array}$ & $\begin{array}{l}.0103 \\
.0092\end{array}$ & $\begin{array}{l}.00022 \\
.00020\end{array}$ & $\begin{array}{l}.24 \\
.21\end{array}$ & $\begin{array}{l}.0031 \\
.0030\end{array}$ & $\begin{array}{l}12.01 \\
12.01\end{array}$ \\
\hline $\begin{array}{l}40.0 \\
40.0 \\
40.0 \\
40.0 \\
40.0\end{array}$ & $\begin{array}{l}40.0 \\
39.0 \\
38.0 \\
37.0 \\
36.0\end{array}$ & $\begin{array}{l}40.0 \\
37.9 \\
35.6 \\
33.2 \\
30.8\end{array}$ & $\begin{array}{r}100.0 \\
92.0 \\
84.2 \\
76.4 \\
68.8\end{array}$ & $\begin{array}{l}.2477 \\
.2279 \\
.2085 \\
.1893 \\
.1705\end{array}$ & $\begin{array}{l}.00541 \\
.00497 \\
.00454 \\
.00412 \\
.00371\end{array}$ & $\begin{array}{l}15.44 \\
14.97 \\
14.51 \\
14.06 \\
13.61\end{array}$ & $\begin{array}{l}.0348 \\
.0338 \\
.0329 \\
.0320 \\
.0311\end{array}$ & $\begin{array}{l}13.17 \\
13.16 \\
13.15 \\
13.14 \\
13.13\end{array}$ \\
\hline $\begin{array}{l}40.0 \\
40.0 \\
40.0 \\
40.0 \\
40.0\end{array}$ & $\begin{array}{l}35.0 \\
34.0 \\
33.0 \\
32.0 \\
31.0\end{array}$ & $\begin{array}{l}28.3 \\
25.6 \\
22.5 \\
21.0 \\
17.4\end{array}$ & $\begin{array}{l}61.4 \\
54.0 \\
46.8 \\
43.5 \\
36.6\end{array}$ & $\begin{array}{l}.1520 \\
.1338 \\
.1158 \\
.1078 \\
.0907\end{array}$ & $\begin{array}{l}.00331 \\
.00291 \\
.00252 \\
.00234 \\
.00197\end{array}$ & $\begin{array}{l}13.18 \\
12.75 \\
12.33 \\
12.14 \\
11.74\end{array}$ & $\begin{array}{l}.0302 \\
.0293 \\
.0284 \\
.0281 \\
.0272\end{array}$ & $\begin{array}{l}13.12 \\
13.11 \\
13.11 \\
13.10 \\
13.09\end{array}$ \\
\hline $\begin{array}{l}40.0 \\
40.0 \\
40.0 \\
40.0 \\
40.0\end{array}$ & $\begin{array}{l}30.0 \\
29.0 \\
28.0 \\
27.0 \\
26.0\end{array}$ & $\begin{array}{r}13.2 \\
8.2 \\
1.7 \\
-7.5 \\
-24.6\end{array}$ & $\begin{array}{r}29.8 \\
23.2 \\
16.6 \\
10.2 \\
3.9\end{array}$ & $\begin{array}{l}.0739 \\
.0574 \\
.0412 \\
.0253 \\
.0097\end{array}$ & $\begin{array}{l}.00160 \\
.00124 \\
.00089 \\
.00055 \\
.00021\end{array}$ & $\begin{array}{r}11.34 \\
10.95 \\
10.57 \\
10.20 \\
9.84\end{array}$ & $\begin{array}{l}.0264 \\
.0256 \\
.0247 \\
.0239 \\
.0231\end{array}$ & $\begin{array}{l}13.09 \\
13.08 \\
13.07 \\
13.06 \\
13.06\end{array}$ \\
\hline
\end{tabular}


$\mathrm{PB}=28.86$, Altitude $=1000-$ Continued

\begin{tabular}{|c|c|c|c|c|c|c|c|c|}
\hline $\mathrm{DB}$ & WB & $\mathrm{DP}$ & $\mathrm{RH}$ & PV & W & $\mathrm{H}$ & S & $\mathrm{V}$ \\
\hline 80.0 & 80.0 & 80.0 & 100.0 & 1.0323 & .02318 & 44.62 & .0907 & 14.62 \\
\hline 80.0 & 79.0 & 78.7 & 95.8 & .9887 & .02217 & 43.51 & .0886 & 14.60 \\
\hline 80.0 & 78.0 & 77.3 & 91.7 & .9461 & .02118 & 42.43 & .0866 & 14.58 \\
\hline 80.0 & 77.0 & 76.0 & 87.6 & .9044 & .02021 & 41.37 & .0846 & 14.56 \\
\hline 80.0 & 76.0 & 74.6 & 83.7 & .8635 & .01927 & 40.34 & .0827 & 14.54 \\
\hline 80.0 & 75.0 & 73.2 & 79.8 & .8236 & .01835 & 39.34 & .0808 & 14.52 \\
\hline 80.0 & 74.0 & 71.7 & 76.0 & .7845 & .01746 & 38.35 & .0790 & 14.50 \\
\hline 80.0 & 73.0 & 70.3 & 72.3 & .7462 & .01658 & 37.39 & .0772 & 14.48 \\
\hline 80.0 & 72.0 & 68.8 & 68.7 & .7087 & .01573 & 36.46 & .0754 & 14.46 \\
\hline 80.0 & 71.0 & 67.2 & 65.1 & .6720 & .01489 & 35.54 & .0737 & 14.44 \\
\hline 80.0 & 70.0 & 65.6 & 61.6 & .6361 & .01408 & 34.65 & .0720 & 14.42 \\
\hline 80.0 & 69.0 & 64.0 & 58.2 & .6009 & .01328 & 33.78 & .0703 & 14.40 \\
\hline 80.0 & 68.0 & 62.3 & 54.9 & .5665 & .01251 & 32.93 & .0687 & 14.38 \\
\hline 80.0 & 67.0 & 60.6 & 51.6 & .5327 & .01175 & 32.10 & .0671 & 14.37 \\
\hline 80.0 & 66.0 & 58.8 & 48.4 & .4997 & .01101 & 31.29 & .0655 & 14.35 \\
\hline 80.0 & 65.0 & 56.9 & 45.3 & .4674 & .01028 & 30.49 & .0640 & 14.33 \\
\hline 80.0 & 64.0 & 55.0 & 42.2 & .4357 & .00957 & 29.72 & .0625 & 14.32 \\
\hline 80.0 & 63.0 & 53.0 & 39.2 & .4047 & .00888 & 28.96 & .0610 & 14.30 \\
\hline 80.0 & 62.0 & 50.9 & 36.3 & .3743 & .00821 & 28.22 & .0595 & 14.29 \\
\hline 80.0 & 61.0 & 48.6 & 33.4 & .3445 & .00755 & 27.49 & .0581 & 14.27 \\
\hline 80.0 & 60.0 & 46.3 & 30.5 & .3153 & .00690 & 26.79 & .0567 & 14.26 \\
\hline 80.0 & 59.0 & 43.8 & 27.8 & .2867 & .00627 & 26.09 & .0554 & 14.24 \\
\hline 80.0 & 58.0 & 41.1 & 25.1 & .2587 & .00565 & 25.42 & .0540 & 14.23 \\
\hline 80.0 & 57.0 & 38.2 & 22.4 & .2312 & .00504 & 24.75 & .0527 & 14.22 \\
\hline 80.0 & 56.0 & 35.1 & 19.8 & .2043 & .00445 & 24.10 & .0514 & 14.20 \\
\hline 80.0 & 55.0 & 31.7 & 17.2 & .1779 & .00387 & 23.47 & .0501 & 14.19 \\
\hline 80.0 & 54.0 & 28.3 & 14.7 & .1520 & .00331 & 22.85 & .0488 & 14.18 \\
\hline 80.0 & 53.0 & 24.4 & 12.3 & .1266 & .00275 & 22.24 & .0476 & 14.16 \\
\hline 80.0 & 52.0 & 19.8 & 9.9 & .1017 & .00221 & 21.64 & .0463 & 14.15 \\
\hline 80.0 & 51.0 & 14.2 & 7.5 & .0773 & .00168 & 21.06 & .0451 & 14.14 \\
\hline 80.0 & 50.0 & 6.8 & 5.2 & .0533 & .00116 & 20.49 & .0439 & 14.13 \\
\hline 80.0 & 49.0 & -4.4 & 2.9 & .0298 & .00065 & 19.93 & .0427 & 14.12 \\
\hline 80.0 & 48.0 & -30.5 & .7 & .0068 & .00015 & 19.38 & .0414 & 14.10 \\
\hline
\end{tabular}


PB $=28.86$, Altitude $=1000-$ Continued

$\begin{array}{rrrrrrrrr}\text { DB } & \text { WB } & \text { DP } & \text { RH } & \text { PV } & \text { W } & \text { H } & \text { S } & \text { V } \\ & & & & & & & & \\ 120.0 & 120.0 & 120.0 & 100.0 & 3.4476 & .08490 & 123.33 & .2311 & 17.21 \\ 120.0 & 118.0 & 117.8 & 94.0 & 3.2410 & .07916 & 116.95 & .2200 & 17.07 \\ 120.0 & 116.0 & 115.5 & 88.3 & 3.0429 & .07374 & 110.92 & .2094 & 16.94 \\ 120.0 & 114.0 & 113.3 & 82.8 & 2.8531 & .06863 & 105.24 & .1995 & 16.81 \\ 120.0 & 112.0 & 111.0 & 77.5 & 2.6714 & .06380 & 99.87 & .1900 & 16.70 \\ 120.0 & 110.0 & 108.7 & 72.4 & 2.4972 & .05925 & 94.80 & .1811 & 16.59 \\ 120.0 & 108.0 & 106.3 & 67.6 & 2.3305 & .05494 & 90.01 & .1726 & 16.48 \\ 120.0 & 106.0 & 103.9 & 63.0 & 2.1708 & .05086 & 85.47 & .1645 & 16.38 \\ 120.0 & 104.0 & 101.4 & 58.5 & 2.0179 & .04700 & 81.18 & .1569 & 16.29 \\ 120.0 & 102.0 & 98.9 & 54.3 & 1.8715 & .04335 & 77.12 & .1496 & 16.20 \\ 120.0 & 100.0 & 96.4 & 50.2 & 1.7314 & .03990 & 73.27 & .1427 & 16.12 \\ 120.0 & 98.0 & 93.7 & 46.3 & 1.5973 & .03662 & 69.63 & .1361 & 16.04 \\ 120.0 & 96.0 & 91.0 & 42.6 & 1.4689 & .03352 & 66.17 & .1298 & 15.96 \\ 120.0 & 94.0 & 88.3 & 39.0 & 1.3461 & .03058 & 62.89 & .1238 & 15.89 \\ 120.0 & 92.0 & 85.4 & 35.6 & 1.2285 & .02778 & 59.79 & .1181 & 15.82 \\ 120.0 & 90.0 & 82.4 & 32.4 & 1.1160 & .02514 & 56.84 & .1127 & 15.76 \\ 120.0 & 88.0 & 79.3 & 29.2 & 1.0083 & .02262 & 54.04 & .1075 & 15.70 \\ 120.0 & 86.0 & 76.0 & 26.3 & .9053 & .02023 & 51.38 & .1025 & 15.64 \\ 120.0 & 84.0 & 72.6 & 23.4 & .8067 & .01797 & 48.85 & .0978 & 15.59 \\ 120.0 & 82.0 & 68.9 & 20.7 & .7123 & .01581 & 46.45 & .0933 & 15.53 \\ 120.0 & 80.0 & 65.0 & 18.0 & .6220 & .01376 & 44.17 & .0889 & 15.48 \\ 120.0 & 78.0 & 60.7 & 15.5 & .5355 & .01181 & 42.00 & .0848 & 15.44 \\ 120.0 & 76.0 & 56.1 & 13.1 & .4527 & .00995 & 39.93 & .0808 & 15.39 \\ 120.0 & 74.0 & 50.8 & 10.8 & .3734 & .00819 & 37.96 & .0770 & 15.35 \\ 120.0 & 72.0 & 44.8 & 8.6 & .2975 & .00651 & 36.09 & .0733 & 15.31 \\ 120.0 & 70.0 & 37.5 & 6.5 & .2248 & .00490 & 34.30 & .0697 & 15.27 \\ 120.0 & 68.0 & 28.7 & 4.5 & .1551 & .00337 & 32.60 & .0663 & 15.23 \\ 120.0 & 66.0 & 16.9 & 2.6 & .0883 & .00192 & 30.98 & .0629 & 15.20 \\ 120.0 & 64.0 & -8.2 & .7 & .0243 & .00053 & 29.43 & .0596 & 15.16\end{array}$


$\mathrm{PB}=28.86$, Altitude $=1000-$ Continued

\begin{tabular}{|c|c|c|c|c|c|c|c|c|}
\hline $\mathrm{DB}$ & WB & $\mathrm{DP}$ & $\mathrm{RH}$ & PV & W & $\mathrm{H}$ & $\mathrm{S}$ & $\mathrm{V}$ \\
\hline 160.0 & 160.0 & 160.0 & 100.0 & 9.6555 & .31557 & 394.96 & .6854 & 24.34 \\
\hline 160.0 & 158.0 & 157.9 & 95.2 & 9.1880 & .29309 & 369.61 & .6438 & 23.77 \\
\hline 160.0 & 156.0 & 155.8 & 90.5 & 8.7380 & .27245 & 346.32 & .6056 & 23.23 \\
\hline 160.0 & 154.0 & 153.8 & 86.0 & 8.3048 & .25344 & 324.87 & .5702 & 22.75 \\
\hline 160.0 & 152.0 & 151.7 & 81.7 & 7.8880 & .23589 & 305.06 & .5375 & 22.29 \\
\hline 160.0 & 150.0 & 149.5 & 77.5 & 7.4870 & .21966 & 286.74 & .5071 & 21.88 \\
\hline 160.0 & 148.0 & 147.4 & 73.5 & 7.1014 & .20462 & 269.76 & .4789 & 21.49 \\
\hline 160.0 & 146.0 & 145.3 & 69.7 & 6.7307 & .19065 & 254.00 & .4526 & 21.13 \\
\hline 160.0 & 144.0 & 143.1 & 66.0 & 6.3744 & .17767 & 239.33 & .4281 & 20.79 \\
\hline 160.0 & 142.0 & 141.0 & 62.5 & 6.0319 & .16557 & 225.68 & .4051 & 20.48 \\
\hline 160.0 & 140.0 & 138.8 & 59.1 & 5.7029 & .15429 & 212.93 & .3837 & 20.19 \\
\hline 160.0 & 138.0 & 136.6 & 55.8 & 5.3869 & .14376 & 201.04 & .3636 & 19.92 \\
\hline 160.0 & 136.0 & 134.4 & 52.6 & 5.0835 & .13391 & 189.91 & .3447 & 19.66 \\
\hline 160.0 & 134.0 & 132.1 & 49.6 & 4.7922 & .12469 & 179.49 & .3270 & 19.43 \\
\hline 160.0 & 132.0 & 129.9 & 46.7 & 4.5126 & .11605 & 169.73 & .3103 & 19.20 \\
\hline 160.0 & 130.0 & 127.6 & 44.0 & 4.2443 & .10795 & 160.57 & .2947 & 18.99 \\
\hline 160.0 & 128.0 & 125.3 & 41.3 & 3.9869 & .10034 & 151.97 & .2799 & 18.80 \\
\hline 160.0 & 126.0 & 122.9 & 38.7 & 3.7400 & .09319 & 143.88 & .2659 & 18.61 \\
\hline 160.0 & 124.0 & 120.6 & 36.3 & 3.5033 & .08646 & 136.28 & .2528 & 18.44 \\
\hline 160.0 & 122.0 & 118.2 & 33.9 & 3.2763 & .08013 & 129.13 & .2404 & 18.27 \\
\hline 160.0 & 120.0 & 115.7 & 31.7 & 3.0587 & .07417 & 122.39 & .2286 & 18.12 \\
\hline 160.0 & 118.0 & 113.2 & 29.5 & 2.8502 & .06855 & 116.03 & .2175 & 17.97 \\
\hline 160.0 & 116.0 & 110.7 & 27.5 & 2.6505 & .06325 & 110.04 & .2069 & 17.84 \\
\hline 160.0 & 114.0 & 108.1 & 25.5 & 2.4591 & .05825 & 104.39 & .1970 & 17.71 \\
\hline 160.0 & 112.0 & 105.5 & 23.6 & 2.2758 & .05353 & 99.05 & .1875 & 17.59 \\
\hline 160.0 & 110.0 & 102.8 & 21.8 & 2.1002 & .04908 & 94.01 & .1785 & 17.47 \\
\hline 160.0 & 108.0 & 100.0 & 20.0 & 1.9322 & .04486 & 89.24 & .1700 & 17.36 \\
\hline 160.0 & 106.0 & 97.1 & 18.3 & 1.7712 & .04088 & 84.73 & .1619 & 17.26 \\
\hline 160.0 & 104.0 & 94.1 & 16.7 & 1.6172 & .03711 & 80.47 & .1542 & 17.16 \\
\hline 160.0 & 102.0 & 91.1 & 15.2 & 1.4698 & .03354 & 76.43 & .1469 & 17.07 \\
\hline 160.0 & 100.0 & 87.8 & 13.8 & 1.3287 & .03016 & 72.61 & .1399 & 16.98 \\
\hline 160.0 & 98.0 & 84.5 & 12.4 & 1.1936 & .02696 & 68.99 & .1333 & 16.90 \\
\hline 160.0 & 96.0 & 80.9 & 11.0 & 1.0644 & .02393 & 65.56 & .1270 & 16.82 \\
\hline 160.0 & 94.0 & 77.2 & 9.7 & .9408 & .02106 & 62.30 & .1209 & 16.74 \\
\hline 160.0 & 92.0 & 73.2 & 8.5 & .8226 & .01833 & 59.22 & .1152 & 16.67 \\
\hline 160.0 & 90.0 & 68.8 & 7.3 & .7094 & .01574 & 56.29 & .1097 & 16.61 \\
\hline 160.0 & 88.0 & 64.0 & 6.2 & .6012 & .01329 & 53.51 & .1044 & 16.54 \\
\hline 160.0 & 86.0 & 58.7 & 5.2 & .4977 & .01096 & 50.88 & .0994 & 16.48 \\
\hline 160.0 & 84.0 & 52.6 & 4.1 & .3986 & .00875 & 48.37 & .0945 & 16.42 \\
\hline 160.0 & 82.0 & 45.3 & 3.1 & .3038 & .00665 & 45.99 & .0898 & 16.37 \\
\hline 160.0 & 80.0 & 36.2 & 2.2 & .2131 & .00465 & 43.73 & .0854 & 16.32 \\
\hline 160.0 & 78.0 & 24.3 & 1.3 & .1263 & .00275 & 41.58 & .0810 & 16.27 \\
\hline 160.0 & 76.0 & 2.7 & .4 & .0433 & .00094 & 39.53 & .0767 & 16.22 \\
\hline
\end{tabular}


$\mathrm{PB}=27.86$, Altitude $=2000$

\begin{tabular}{|c|c|c|c|c|c|c|c|c|}
\hline DB & WB & $\mathrm{DP}$ & $\mathrm{RH}$ & PV & W & $\mathrm{H}$ & S & V \\
\hline-40.0 & -40.0 & -40.0 & 100.0 & .0038 & .00009 & -9.51 & -.0167 & 11.35 \\
\hline $\begin{array}{l}.0 \\
.0 \\
.0 \\
.0 \\
.0\end{array}$ & $\begin{array}{r}.0 \\
-.1 \\
-.2 \\
-.3 \\
-.4\end{array}$ & $\begin{array}{r}.0 \\
-.6 \\
-1.1 \\
-1.7 \\
-2.3\end{array}$ & $\begin{array}{r}100.0 \\
97.1 \\
94.3 \\
91.4 \\
88.6\end{array}$ & $\begin{array}{l}.0376 \\
.0366 \\
.0355 \\
.0344 \\
.0333\end{array}$ & $\begin{array}{l}.00085 \\
.00082 \\
.00080 \\
.00077 \\
.00075\end{array}$ & $\begin{array}{l}.90 \\
.88 \\
.85 \\
.83 \\
.80\end{array}$ & $\begin{array}{l}.0070 \\
.0069 \\
.0069 \\
.0068 \\
.0067\end{array}$ & $\begin{array}{l}12.45 \\
12.45 \\
12.45 \\
12.45 \\
12.45\end{array}$ \\
\hline $\begin{array}{l}.0 \\
.0 \\
.0 \\
.0 \\
.0\end{array}$ & $\begin{array}{l}-.5 \\
-.6 \\
-.7 \\
-.8 \\
-.9\end{array}$ & $\begin{array}{l}-2.9 \\
-3.5 \\
-4.2 \\
-4.9 \\
-5.6\end{array}$ & $\begin{array}{l}85.8 \\
82.9 \\
80.1 \\
77.2 \\
74.4\end{array}$ & $\begin{array}{l}.0323 \\
.0312 \\
.0301 \\
.0291 \\
.0280\end{array}$ & $\begin{array}{l}.00072 \\
.00070 \\
.00068 \\
.00065 \\
.00063\end{array}$ & $\begin{array}{l}.78 \\
.75 \\
.73 \\
.70 \\
.67\end{array}$ & $\begin{array}{l}.0067 \\
.0066 \\
.0066 \\
.0065 \\
.0065\end{array}$ & $\begin{array}{l}12.45 \\
12.45 \\
12.45 \\
12.45 \\
12.45\end{array}$ \\
\hline $\begin{array}{l}.0 \\
.0 \\
.0 \\
.0 \\
.0\end{array}$ & $\begin{array}{l}-1.0 \\
-1.1 \\
-1.2 \\
-1.3 \\
-1.4\end{array}$ & $\begin{array}{l}-6.3 \\
-7.0 \\
-7.8 \\
-8.6 \\
-9.5\end{array}$ & $\begin{array}{l}71.6 \\
68.7 \\
65.9 \\
63.1 \\
60.3\end{array}$ & $\begin{array}{l}.0269 \\
.0259 \\
.0248 \\
.0237 \\
.0227\end{array}$ & $\begin{array}{l}.00060 \\
.00058 \\
.00056 \\
.00053 \\
.00051\end{array}$ & $\begin{array}{l}.65 \\
.62 \\
.60 \\
.57 \\
.55\end{array}$ & $\begin{array}{l}.0064 \\
.0064 \\
.0063 \\
.0062 \\
.0062\end{array}$ & $\begin{array}{l}12.45 \\
12.45 \\
12.45 \\
12.45 \\
12.45\end{array}$ \\
\hline $\begin{array}{l}.0 \\
.0 \\
.0 \\
.0 \\
.0\end{array}$ & $\begin{array}{l}-1.5 \\
-1.6 \\
-1.7 \\
-1.8 \\
-1.9\end{array}$ & $\begin{array}{l}-10.3 \\
-11.3 \\
-12.2 \\
-13.2 \\
-14.3\end{array}$ & $\begin{array}{l}57.4 \\
54.6 \\
51.8 \\
49.0 \\
46.2\end{array}$ & $\begin{array}{l}.0216 \\
.0206 \\
.0195 \\
.0184 \\
.0174\end{array}$ & $\begin{array}{l}.00049 \\
.00046 \\
.00044 \\
.00041 \\
.00039\end{array}$ & $\begin{array}{l}.52 \\
.50 \\
.47 \\
.45 \\
.42\end{array}$ & $\begin{array}{l}.0061 \\
.0061 \\
.0060 \\
.0060 \\
.0059\end{array}$ & $\begin{array}{l}12.45 \\
12.44 \\
12.44 \\
12.44 \\
12.44\end{array}$ \\
\hline $\begin{array}{l}.0 \\
.0 \\
.0 \\
.0 \\
.0\end{array}$ & $\begin{array}{l}-2.0 \\
-2.1 \\
-2.2 \\
-2.3 \\
-2.4\end{array}$ & $\begin{array}{l}-15.4 \\
-16.6 \\
-17.9 \\
-19.2 \\
-20.7\end{array}$ & $\begin{array}{l}43.4 \\
40.6 \\
37.8 \\
35.0 \\
32.2\end{array}$ & $\begin{array}{l}.0163 \\
.0153 \\
.0142 \\
.0132 \\
.0121\end{array}$ & $\begin{array}{l}.00037 \\
.00034 \\
.00032 \\
.00030 \\
.00027\end{array}$ & $\begin{array}{l}.40 \\
.37 \\
.35 \\
.32 \\
.30\end{array}$ & $\begin{array}{l}.0058 \\
.0058 \\
.0057 \\
.0057 \\
.0056\end{array}$ & $\begin{array}{l}12.44 \\
12.44 \\
12.44 \\
12.44 \\
12.44\end{array}$ \\
\hline $\begin{array}{l}.0 \\
.0 \\
.0 \\
.0\end{array}$ & $\begin{array}{l}-2.5 \\
-2.6 \\
-2.7 \\
-2.8\end{array}$ & $\begin{array}{l}-22.2 \\
-24.0 \\
-25.8 \\
-28.0\end{array}$ & $\begin{array}{l}29.4 \\
26.6 \\
23.8 \\
21.0\end{array}$ & $\begin{array}{l}.0111 \\
.0100 \\
.0090 \\
.0079\end{array}$ & $\begin{array}{l}.00025 \\
.00022 \\
.00020 \\
.00018\end{array}$ & $\begin{array}{l}.27 \\
.25 \\
.22 \\
.20\end{array}$ & $\begin{array}{l}.0056 \\
.0055 \\
.0054 \\
.0054\end{array}$ & $\begin{array}{l}12.44 \\
12.44 \\
12.44 \\
12.44\end{array}$ \\
\hline $\begin{array}{l}40.0 \\
40.0 \\
40.0 \\
40.0 \\
40.0\end{array}$ & $\begin{array}{l}40.0 \\
39.0 \\
38.0 \\
37.0 \\
36.0\end{array}$ & $\begin{array}{l}40.0 \\
37.9 \\
35.7 \\
33.3 \\
31.0\end{array}$ & $\begin{array}{r}100.0 \\
92.2 \\
84.5 \\
76.9 \\
69.4\end{array}$ & $\begin{array}{l}.2477 \\
.2283 \\
.2092 \\
.1904 \\
.1719\end{array}$ & $\begin{array}{l}.00560 \\
.00516 \\
.00472 \\
.00430 \\
.00388\end{array}$ & $\begin{array}{l}15.66 \\
15.18 \\
14.71 \\
14.25 \\
13.80\end{array}$ & $\begin{array}{l}.0377 \\
.0367 \\
.0358 \\
.0348 \\
.0339\end{array}$ & $\begin{array}{l}13.64 \\
13.63 \\
13.62 \\
13.62 \\
13.61\end{array}$ \\
\hline $\begin{array}{l}40.0 \\
40.0 \\
40.0 \\
40.0 \\
40.0\end{array}$ & $\begin{array}{l}35.0 \\
34.0 \\
33.0 \\
32.0 \\
31.0\end{array}$ & $\begin{array}{l}28.5 \\
25.9 \\
23.0 \\
21.5 \\
18.1\end{array}$ & $\begin{array}{l}62.1 \\
54.9 \\
47.8 \\
44.6 \\
37.8\end{array}$ & $\begin{array}{l}.1538 \\
.1359 \\
.1184 \\
.1104 \\
.0935\end{array}$ & $\begin{array}{l}.00347 \\
.00306 \\
.00266 \\
.00248 \\
.00210\end{array}$ & $\begin{array}{l}13.35 \\
12.92 \\
12.49 \\
12.29 \\
11.88\end{array}$ & $\begin{array}{l}.0330 \\
.0321 \\
.0312 \\
.0308 \\
.0300\end{array}$ & $\begin{array}{l}13.60 \\
13.59 \\
13.58 \\
13.58 \\
13.57\end{array}$ \\
\hline $\begin{array}{l}40.0 \\
40.0 \\
40.0 \\
40.0 \\
40.0\end{array}$ & $\begin{array}{l}30.0 \\
29.0 \\
28.0 \\
27.0 \\
26.0\end{array}$ & $\begin{array}{r}14.1 \\
9.4 \\
3.4 \\
-4.7 \\
-18.0\end{array}$ & $\begin{array}{r}31.1 \\
24.6 \\
18.2 \\
11.9 \\
5.7\end{array}$ & $\begin{array}{l}.0770 \\
.0608 \\
.0450 \\
.0294 \\
.0141\end{array}$ & $\begin{array}{l}.00173 \\
.00137 \\
.00101 \\
.00066 \\
.00032\end{array}$ & $\begin{array}{r}11.48 \\
11.09 \\
10.70 \\
10.33 \\
9.96\end{array}$ & $\begin{array}{l}.0291 \\
.0283 \\
.0274 \\
.0266 \\
.0258\end{array}$ & $\begin{array}{l}13.56 \\
13.55 \\
13.54 \\
13.54 \\
13.53\end{array}$ \\
\hline
\end{tabular}


$\mathrm{PB}=27.86$, Altitude $=2000-$ Continued

\begin{tabular}{|c|c|c|c|c|c|c|c|c|}
\hline $\mathrm{DB}$ & WB & $\mathrm{DP}$ & $\mathrm{RH}$ & PV & W & $\mathrm{H}$ & $\mathrm{S}$ & $\mathrm{V}$ \\
\hline 80.0 & 80.0 & 80.0 & 100.0 & 1.0323 & .02404 & 45.57 & .0950 & 15.17 \\
\hline 80.0 & 79.0 & 78.7 & 95.8 & .9891 & .02300 & 44.43 & .0928 & 15.15 \\
\hline 80.0 & 78.0 & 77.4 & 91.7 & .9468 & .02198 & 43.31 & .0908 & 15.12 \\
\hline 80.0 & 77.0 & 76.0 & 87.7 & .9055 & .02099 & 42.22 & .0887 & 15.10 \\
\hline 80.0 & 76.0 & 74.7 & 83.8 & .8650 & .02002 & 41.16 & .0867 & 15.08 \\
\hline 80.0 & 75.0 & 73.3 & 80.0 & .8254 & .01907 & 40.13 & .0848 & 15.05 \\
\hline 80.0 & 74.0 & 71.8 & 76.2 & .7866 & 01815 & 39.12 & .0829 & 15.03 \\
\hline 80.0 & 73.0 & 70.4 & 72.5 & .7487 & .01725 & 38.13 & .0810 & 15.01 \\
\hline 80.0 & 72.0 & 68.9 & 68.9 & .7116 & .01637 & 37.17 & .0792 & 14.99 \\
\hline 80.0 & 71.0 & 67.4 & 65.4 & .6753 & .01552 & 36.23 & .0774 & 14.97 \\
\hline 80.0 & 70.0 & 65.8 & 62.0 & .6397 & .01468 & 35.31 & .0757 & 14.95 \\
\hline 80.0 & 69.0 & 64.2 & 58.6 & .6049 & .01386 & 34.42 & .0740 & 14.93 \\
\hline 80.0 & 68.0 & 62.5 & 55.3 & .5708 & .01307 & 33.55 & .0723 & 14.91 \\
\hline 80.0 & 67.0 & 60.8 & 52.1 & .5375 & .01229 & 32.69 & .0707 & 14.90 \\
\hline 80.0 & 66.0 & 59.1 & 48.9 & .5048 & .01153 & 31.86 & .0691 & 14.88 \\
\hline 80.0 & 65.0 & 57.3 & 45.8 & .4728 & .01078 & 31.05 & .0675 & 14.86 \\
\hline 80.0 & 64.0 & 55.4 & 42.8 & .4415 & .01006 & 30.25 & .0660 & 14.84 \\
\hline 80.0 & 63.0 & 53.4 & 39.8 & .4108 & .00935 & 29.47 & .0645 & 14.83 \\
\hline 80.0 & 62.0 & 51.3 & 36.9 & .3808 & .00866 & 28.71 & .0630 & 14.81 \\
\hline 80.0 & 61.0 & 49.2 & 34.0 & .3514 & .00798 & 27.97 & .0615 & 14.79 \\
\hline 80.0 & 60.0 & 46.9 & 31.2 & .3226 & .00732 & 27.24 & .0601 & 14.78 \\
\hline 80.0 & 59.0 & 44.5 & 28.5 & .2943 & .00667 & 26.54 & .0587 & 14.76 \\
\hline 80.0 & 58.0 & 41.9 & 25.8 & .2667 & .00604 & 25.84 & .0573 & 14.75 \\
\hline 80.0 & 57.0 & 39.1 & 23.2 & .2395 & .00542 & 25.16 & .0559 & 14.73 \\
\hline 80.0 & 56.0 & 36.2 & 20.6 & .2130 & .00481 & 24.50 & .0546 & 14.72 \\
\hline 80.0 & 55.0 & 32.9 & 18.1 & .1869 & .00422 & 23.85 & .0533 & 14.71 \\
\hline 80.0 & 54.0 & 29.6 & 15.6 & .1614 & .00364 & 23.21 & .0520 & 14.69 \\
\hline 80.0 & 53.0 & 26.0 & 13.2 & .1364 & .00307 & 22.59 & .0507 & 14.68 \\
\hline 80.0 & 52.0 & 21.8 & 10.8 & .1118 & .00252 & 21.98 & .0495 & 14.67 \\
\hline 80.0 & 51.0 & 16.7 & 8.5 & .0878 & .00197 & 21.39 & .0482 & 14.65 \\
\hline 80.0 & 50.0 & 10.4 & 6.2 & .0642 & .00144 & 20.81 & .0470 & 14.64 \\
\hline 80.0 & 49.0 & 1.6 & 4.0 & .0410 & .00092 & 20.23 & .0458 & 14.63 \\
\hline 80.0 & 48.0 & -13.4 & 1.8 & .0183 & .00041 & 19.68 & .0445 & 14.62 \\
\hline
\end{tabular}


PB $=27.86$, Altitude $=2000-$ Continued

$\begin{array}{rrrrrrrrr}\text { DB } & \text { WB } & \text { DP } & \text { RH } & \text { PV } & \text { W } & \text { H } & \text { S } & \text { V } \\ 120.0 & 120.0 & 120.0 & 100.0 & 3.4476 & .08837 & 127.20 & .2405 & 17.91 \\ 120.0 & 118.0 & 117.8 & 94.0 & 3.2417 & .08239 & 120.54 & .2289 & 17.76 \\ 120.0 & 116.0 & 115.6 & 88.3 & 3.0444 & .07675 & 114.27 & .2180 & 17.62 \\ 120.0 & 114.0 & 113.3 & 82.8 & 2.8554 & .07143 & 108.36 & .2076 & 17.49 \\ 120.0 & 112.0 & 111.0 & 77.6 & 2.6743 & .06641 & 102.78 & .1978 & 17.36 \\ 120.0 & 110.0 & 108.7 & 72.5 & 2.5009 & .06168 & 97.51 & .1885 & 17.24 \\ 120.0 & 108.0 & 106.4 & 67.7 & 2.3349 & .05720 & 92.53 & .1797 & 17.13 \\ 120.0 & 106.0 & 104.0 & 63.1 & 2.1760 & .05297 & 87.83 & .1713 & 17.02 \\ 120.0 & 104.0 & 101.5 & 58.7 & 2.0238 & .04897 & 83.38 & .1634 & 16.92 \\ 120.0 & 102.0 & 99.0 & 54.5 & 1.8782 & .04519 & 79.17 & .1558 & 16.83 \\ 120.0 & 100.0 & 96.5 & 50.4 & 1.7388 & .04161 & 75.18 & .1487 & 16.74 \\ 120.0 & 98.0 & 93.9 & 46.6 & 1.6054 & .03822 & 71.41 & .1419 & 16.65 \\ 120.0 & 96.0 & 91.2 & 42.9 & 1.4778 & .03501 & 67.83 & .1354 & 16.57 \\ 120.0 & 94.0 & 88.5 & 39.3 & 1.3557 & .03196 & 64.44 & .1292 & 16.50 \\ 120.0 & 92.0 & 85.6 & 35.9 & 1.2388 & .02908 & 61.23 & .1233 & 16.42 \\ 120.0 & 90.0 & 82.7 & 32.7 & 1.1270 & .02634 & 58.18 & .1177 & 16.36 \\ 120.0 & 88.0 & 79.6 & 29.6 & 1.0201 & .02375 & 55.29 & .1123 & 16.29 \\ 120.0 & 86.0 & 76.4 & 26.6 & .9178 & .02128 & 52.55 & .1072 & 16.23 \\ 120.0 & 84.0 & 73.1 & 23.8 & .8199 & .01894 & 49.94 & .1023 & 16.17 \\ 120.0 & 82.0 & 69.5 & 21.1 & .7262 & .01672 & 47.47 & .0977 & 16.11 \\ 120.0 & 80.0 & 65.7 & 18.5 & .6366 & .01461 & 45.12 & .0932 & 16.06 \\ 120.0 & 78.0 & 61.5 & 16.0 & .5509 & .01260 & 42.88 & .0889 & 16.01 \\ 120.0 & 76.0 & 57.0 & 13.6 & .4688 & .01069 & 40.75 & .0848 & 15.96 \\ 120.0 & 74.0 & 52.0 & 11.3 & .3902 & .00887 & 38.73 & .0809 & 15.92 \\ 120.0 & 72.0 & 46.3 & 9.1 & .3150 & .00714 & 36.80 & .0771 & 15.87 \\ 120.0 & 70.0 & 39.5 & 7.0 & .2430 & .00549 & 34.96 & .0735 & 15.83 \\ 120.0 & 68.0 & 31.2 & 5.0 & .1740 & .00392 & 33.22 & .0700 & 15.79 \\ 120.0 & 66.0 & 21.0 & 3.1 & .1079 & .00243 & 31.55 & .0665 & 15.75 \\ 120.0 & 64.0 & 3.3 & 1.3 & .0446 & .00100 & 29.96 & .0632 & 15.72\end{array}$


$\mathrm{PB}=27.86$, Altitude $=2000-$ Continued

\begin{tabular}{|c|c|c|c|c|c|c|c|c|}
\hline $\mathrm{DB}$ & WB & $\mathrm{DP}$ & $\mathrm{RH}$ & PV & W & $\mathrm{H}$ & $\mathrm{S}$ & $\mathrm{V}$ \\
\hline 160.0 & 160.0 & 160.0 & 100.0 & 9.6555 & .33287 & 414.52 & .7210 & 25.68 \\
\hline 160.0 & 158.0 & 157.9 & 95.2 & 9.1888 & .30880 & 387.36 & .6765 & 25.04 \\
\hline 160.0 & 156.0 & 155.9 & 90.5 & 8.7395 & .28675 & 362.48 & .6357 & 24.45 \\
\hline 160.0 & 154.0 & 153.8 & 86.0 & 8.3071 & .26648 & 339.61 & .5980 & 23.91 \\
\hline 160.0 & 152.0 & 151.7 & 81.7 & 7.8911 & .24782 & 318.55 & .5632 & 23.41 \\
\hline 160.0 & 150.0 & 149.6 & 77.6 & 7.4909 & .23058 & 299.10 & .5309 & 22.95 \\
\hline 160.0 & 148.0 & 147.4 & 73.6 & 7.1061 & .21464 & 281.10 & .5010 & 22.53 \\
\hline 160.0 & 146.0 & 145.3 & 69.8 & 6.7361 & .19987 & 264.42 & .4732 & 22.13 \\
\hline 160.0 & 144.0 & 143.2 & 66.1 & 6.3805 & .18615 & 248.93 & .4473 & 21.77 \\
\hline 160.0 & 142.0 & 141.0 & 62.5 & 6.0388 & .17340 & 234.52 & .4231 & 21.43 \\
\hline 160.0 & 140.0 & 138.8 & 59.1 & 5.7105 & .16151 & 221.10 & .4005 & 21.11 \\
\hline 160.0 & 138.0 & 136.6 & 55.9 & 5.3953 & .15043 & 208.59 & .3793 & 20.81 \\
\hline 160.0 & 136.0 & 134.4 & 52.7 & 5.0926 & .14008 & 196.89 & .3595 & 20.54 \\
\hline 160.0 & 134.0 & 132.2 & 49.7 & 4.8020 & .13041 & 185.96 & .3409 & 20.28 \\
\hline 160.0 & 132.0 & 130.0 & 46.8 & 4.5232 & .12135 & 175.72 & .3235 & 20.03 \\
\hline 160.0 & 130.0 & 127.7 & 44.1 & 4.2556 & .11286 & 166.13 & .3070 & 19.81 \\
\hline 160.0 & 128.0 & 125.4 & 41.4 & 3.9990 & .10490 & 157.13 & .2916 & 19.59 \\
\hline 160.0 & 126.0 & 123.1 & 38.9 & 3.7528 & .09742 & 148.68 & .2770 & 19.39 \\
\hline 160.0 & 124.0 & 120.7 & 36.4 & 3.5168 & .09040 & 140.74 & .2633 & 19.21 \\
\hline 160.0 & 122.0 & 118.3 & 34.1 & 3.2906 & .08380 & 133.27 & .2503 & 19.03 \\
\hline 160.0 & 120.0 & 115.9 & 31.8 & 3.0737 & .07758 & 126.25 & .2381 & 18.86 \\
\hline 160.0 & 118.0 & 113.4 & 29.7 & 2.8660 & .07173 & 119.63 & .2265 & 18.70 \\
\hline 160.0 & 116.0 & 110.9 & 27.6 & 2.6669 & .06621 & 113.39 & .2155 & 18.56 \\
\hline 160.0 & 114.0 & 108.4 & 25.6 & 2.4763 & .06101 & 107.50 & .2051 & 18.42 \\
\hline 160.0 & 112.0 & 105.7 & 23.8 & 2.2937 & .05610 & 101.95 & .1953 & 18.29 \\
\hline 160.0 & 110.0 & 103.1 & 21.9 & 2.1188 & .05147 & 96.71 & .1860 & 18.16 \\
\hline 160.0 & 108.0 & 100.3 & 20.2 & 1.9515 & .04709 & 91.76 & .1771 & 18.04 \\
\hline 160.0 & 106.0 & 97.5 & 18.6 & 1.7913 & .04295 & 87.08 & .1687 & 17.93 \\
\hline 160.0 & 104.0 & 94.6 & 17.0 & 1.6380 & .03904 & 82.66 & .1608 & 17.83 \\
\hline 160.0 & 102.0 & 91.5 & 15.4 & 1.4912 & .03534 & 78.48 & .1532 & 17.73 \\
\hline 160.0 & 100.0 & 88.4 & 14.0 & 1.3508 & .03184 & 74.52 & .1460 & 17.63 \\
\hline 160.0 & 98.0 & 85.1 & 12.6 & 1.2165 & .02853 & 70.77 & .1391 & 17.54 \\
\hline 160.0 & 96.0 & 81.6 & 11.3 & 1.0881 & .02539 & 67.22 & .1326 & 17.46 \\
\hline 160.0 & 94.0 & 78.0 & 10.0 & .9652 & .02242 & 63.85 & .1263 & 17.38 \\
\hline 160.0 & 92.0 & 74.0 & 8.8 & .8476 & .01960 & 60.66 & .1204 & 17.30 \\
\hline 160.0 & 90.0 & 69.8 & 7.6 & .7352 & .01693 & 57.64 & .1147 & 17.23 \\
\hline 160.0 & 88.0 & 65.3 & 6.5 & .6276 & .01440 & 54.77 & .1093 & 17.16 \\
\hline 160.0 & 86.0 & 60.2 & 5.4 & .5248 & .01199 & 52.05 & .1041 & 17.10 \\
\hline 160.0 & 84.0 & 54.4 & 4.4 & .4264 & .00971 & 49.46 & .0991 & 17.04 \\
\hline 160.0 & 82.0 & 47.7 & 3.4 & .3324 & .00754 & 47.01 & .0943 & 16.98 \\
\hline 160.0 & 80.0 & 39.4 & 2.5 & .2424 & .00548 & 44.68 & .0897 & 16.92 \\
\hline 160.0 & 78.0 & 28.9 & 1.6 & .1563 & .00352 & 42.46 & .0852 & 16.87 \\
\hline 160.0 & 76.0 & 13.3 & .8 & .0739 & .00166 & 40.35 & .0809 & 16.82 \\
\hline
\end{tabular}


PB $=26.87$, Altitude $=3000$

\begin{tabular}{|c|c|c|c|c|c|c|c|c|}
\hline $\mathrm{DB}$ & WB & $\mathrm{DP}$ & $\mathrm{RH}$ & PV & W & $\mathrm{H}$ & S & V \\
\hline-40.0 & -40.0 & -40.0 & 100.0 & .0038 & .00009 & -9.50 & -.0142 & 11.77 \\
\hline $\begin{array}{l}.0 \\
.0 \\
.0 \\
.0 \\
.0\end{array}$ & $\begin{array}{r}.0 \\
-.1 \\
-.2 \\
-.3 \\
-.4\end{array}$ & $\begin{array}{r}.0 \\
-.5 \\
-1.1 \\
-1.6 \\
-2.2\end{array}$ & $\begin{array}{r}100.0 \\
97.2 \\
94.5 \\
91.7 \\
88.9\end{array}$ & $\begin{array}{l}.0376 \\
.0366 \\
.0356 \\
.0345 \\
.0335\end{array}$ & $\begin{array}{l}.00088 \\
.00085 \\
.00083 \\
.00080 \\
.00078\end{array}$ & $\begin{array}{l}.94 \\
.92 \\
.89 \\
.86 \\
.84\end{array}$ & $\begin{array}{l}.0095 \\
.0095 \\
.0094 \\
.0094 \\
.0093\end{array}$ & $\begin{array}{l}12.91 \\
12.91 \\
12.91 \\
12.91 \\
12.91\end{array}$ \\
\hline $\begin{array}{l}.0 \\
.0 \\
.0 \\
.0 \\
.0\end{array}$ & $\begin{array}{l}-.5 \\
-.6 \\
-.7 \\
-.8 \\
-.9\end{array}$ & $\begin{array}{l}-2.8 \\
-3.4 \\
-4.1 \\
-4.7 \\
-5.4\end{array}$ & $\begin{array}{l}86.2 \\
83.4 \\
80.7 \\
77.9 \\
75.1\end{array}$ & $\begin{array}{l}.0324 \\
.0314 \\
.0304 \\
.0293 \\
.0283\end{array}$ & $\begin{array}{l}.00075 \\
.00073 \\
.00071 \\
.00068 \\
.00066\end{array}$ & $\begin{array}{l}.81 \\
.79 \\
.76 \\
.74 \\
.71\end{array}$ & $\begin{array}{l}.0093 \\
.0092 \\
.0091 \\
.0091 \\
.0090\end{array}$ & $\begin{array}{l}12.91 \\
12.91 \\
12.91 \\
12.91 \\
12.91\end{array}$ \\
\hline $\begin{array}{l}.0 \\
.0 \\
.0 \\
.0 \\
.0\end{array}$ & $\begin{array}{l}-1.0 \\
-1.1 \\
-1.2 \\
-1.3 \\
-1.4\end{array}$ & $\begin{array}{l}-6.1 \\
-6.8 \\
-7.5 \\
-8.3 \\
-9.1\end{array}$ & $\begin{array}{l}72.4 \\
69.7 \\
66.9 \\
64.2 \\
61.4\end{array}$ & $\begin{array}{l}.0273 \\
.0262 \\
.0252 \\
.0242 \\
.0231\end{array}$ & $\begin{array}{l}.00063 \\
.00061 \\
.00059 \\
.00056 \\
.00054\end{array}$ & $\begin{array}{l}.68 \\
.66 \\
.63 \\
.61 \\
.58\end{array}$ & $\begin{array}{l}.0090 \\
.0089 \\
.0089 \\
.0088 \\
.0087\end{array}$ & $\begin{array}{c}12.91 \\
12.91 \\
12.91 \\
12.91 \\
12.91\end{array}$ \\
\hline $\begin{array}{l}.0 \\
.0 \\
.0 \\
.0 \\
.0\end{array}$ & $\begin{array}{l}-1.5 \\
-1.6 \\
-1.7 \\
-1.8 \\
-1.9\end{array}$ & $\begin{array}{l}-10.0 \\
-10.8 \\
-11.7 \\
-12.7 \\
-13.7\end{array}$ & $\begin{array}{l}58.7 \\
56.0 \\
53.2 \\
50.5 \\
47.8\end{array}$ & $\begin{array}{l}.0221 \\
.0211 \\
.0200 \\
.0190 \\
.0180\end{array}$ & $\begin{array}{l}.00051 \\
.00049 \\
.00047 \\
.00044 \\
.00042\end{array}$ & $\begin{array}{l}.56 \\
.53 \\
.51 \\
.48 \\
.46\end{array}$ & $\begin{array}{l}.0087 \\
.0086 \\
.0086 \\
.0085 \\
.0085\end{array}$ & $\begin{array}{l}12.90 \\
12.90 \\
12.90 \\
12.90 \\
12.90\end{array}$ \\
\hline $\begin{array}{l}.0 \\
.0 \\
.0 \\
.0 \\
.0\end{array}$ & $\begin{array}{l}-2.0 \\
-2.1 \\
-2.2 \\
-2.3 \\
-2.4\end{array}$ & $\begin{array}{l}-14.7 \\
-15.8 \\
-17.0 \\
-18.3 \\
-19.6\end{array}$ & $\begin{array}{l}45.0 \\
42.3 \\
39.6 \\
36.9 \\
34.2\end{array}$ & $\begin{array}{l}.0170 \\
.0159 \\
.0149 \\
.0139 \\
.0129\end{array}$ & $\begin{array}{l}.00039 \\
.00037 \\
.00035 \\
.00032 \\
.00030\end{array}$ & $\begin{array}{l}.43 \\
.41 \\
.38 \\
.35 \\
.33\end{array}$ & $\begin{array}{l}.0084 \\
.0083 \\
.0083 \\
.0082 \\
.0082\end{array}$ & $\begin{array}{l}12.90 \\
12.90 \\
12.90 \\
12.90 \\
12.90\end{array}$ \\
\hline $\begin{array}{l}.0 \\
.0 \\
.0 \\
.0 \\
.0\end{array}$ & $\begin{array}{l}-2.5 \\
-2.6 \\
-2.7 \\
-2.8 \\
-2.9\end{array}$ & $\begin{array}{l}-21.1 \\
-22.6 \\
-24.3 \\
-26.2 \\
-28.3\end{array}$ & $\begin{array}{l}31.5 \\
28.8 \\
26.0 \\
23.3 \\
20.6\end{array}$ & $\begin{array}{l}.0118 \\
.0108 \\
.0098 \\
.0088 \\
.0078\end{array}$ & $\begin{array}{l}.00028 \\
.00025 \\
.00023 \\
.00020 \\
.00018\end{array}$ & $\begin{array}{l}.30 \\
.28 \\
.25 \\
.23 \\
.20\end{array}$ & $\begin{array}{l}.0081 \\
.0080 \\
.0080 \\
.0079 \\
.0079\end{array}$ & $\begin{array}{l}12.90 \\
12.90 \\
12.90 \\
12.90 \\
12.90\end{array}$ \\
\hline $\begin{array}{l}40.0 \\
40.0 \\
40.0 \\
40.0 \\
40.0\end{array}$ & $\begin{array}{l}40.0 \\
39.0 \\
38.0 \\
37.0 \\
36.0\end{array}$ & $\begin{array}{l}40.0 \\
38.0 \\
35.8 \\
33.5 \\
31.1\end{array}$ & $\begin{array}{r}100.0 \\
92.3 \\
84.7 \\
77.3 \\
70.0\end{array}$ & $\begin{array}{l}.2477 \\
.2286 \\
.2099 \\
.1915 \\
.1734\end{array}$ & $\begin{array}{l}.00581 \\
.00536 \\
.00492 \\
.00448 \\
.00406\end{array}$ & $\begin{array}{l}15.88 \\
15.40 \\
14.92 \\
14.45 \\
13.99\end{array}$ & $\begin{array}{l}.0406 \\
.0396 \\
.0387 \\
.0377 \\
.0368\end{array}$ & $\begin{array}{l}14.15 \\
14.14 \\
14.13 \\
14.12 \\
14.11\end{array}$ \\
\hline $\begin{array}{l}40.0 \\
40.0 \\
40.0 \\
40.0 \\
40.0\end{array}$ & $\begin{array}{l}35.0 \\
34.0 \\
33.0 \\
32.0 \\
31.0\end{array}$ & $\begin{array}{l}28.8 \\
26.2 \\
23.4 \\
22.0 \\
18.7\end{array}$ & $\begin{array}{l}62.8 \\
55.7 \\
48.8 \\
45.6 \\
38.9\end{array}$ & $\begin{array}{l}.1556 \\
.1381 \\
.1208 \\
.1129 \\
.0964\end{array}$ & $\begin{array}{l}.00364 \\
.00323 \\
.00282 \\
.00263 \\
.00225\end{array}$ & $\begin{array}{l}13.54 \\
13.10 \\
12.66 \\
12.46 \\
12.04\end{array}$ & $\begin{array}{l}.0359 \\
.0350 \\
.0341 \\
.0336 \\
.0328\end{array}$ & $\begin{array}{l}14.10 \\
14.09 \\
14.08 \\
14.08 \\
14.07\end{array}$ \\
\hline $\begin{array}{l}40.0 \\
40.0 \\
40.0 \\
40.0 \\
40.0 \\
40.0\end{array}$ & $\begin{array}{l}30.0 \\
29.0 \\
28.0 \\
27.0 \\
26.0 \\
25.0\end{array}$ & $\begin{array}{r}14.9 \\
10.4 \\
5.0 \\
-2.3 \\
-13.2 \\
-40.3\end{array}$ & $\begin{array}{r}32.4 \\
26.0 \\
19.7 \\
13.5 \\
7.4 \\
1.5\end{array}$ & $\begin{array}{l}.0802 \\
.0643 \\
.0487 \\
.0334 \\
.0184 \\
.0037\end{array}$ & $\begin{array}{l}.00187 \\
.00150 \\
.00113 \\
.00078 \\
.00043 \\
.00009\end{array}$ & $\begin{array}{r}11.63 \\
11.23 \\
10.84 \\
10.46 \\
10.08 \\
9.71\end{array}$ & $\begin{array}{l}.0319 \\
.0311 \\
.0302 \\
.0294 \\
.0285 \\
.0277\end{array}$ & $\begin{array}{l}14.06 \\
14.05 \\
14.05 \\
14.04 \\
14.03 \\
14.02\end{array}$ \\
\hline
\end{tabular}


$\mathrm{PB}=26.87$, Altitude $=3000$ - Continued

\begin{tabular}{|c|c|c|c|c|c|c|c|c|}
\hline $\mathrm{DB}$ & WB & $\mathrm{DP}$ & $\mathrm{RH}$ & PV & W & $\mathrm{H}$ & $\mathrm{S}$ & V \\
\hline 80.0 & 80.0 & 80.0 & 100.0 & 1.0323 & .02496 & 46.58 & .0994 & 15.75 \\
\hline 80.0 & 79.0 & 78.7 & 95.9 & .9894 & .02388 & 45.40 & .0972 & 15.73 \\
\hline 80.0 & 78.0 & 77.4 & 91.8 & .9475 & .02283 & 44.25 & .0951 & 15.70 \\
\hline 80.0 & 77.0 & 76.1 & 87.8 & .9065 & .02181 & 43.13 & .0930 & 15.68 \\
\hline 80.0 & 76.0 & 74.7 & 83.9 & .8664 & .02081 & 42.04 & .0909 & 15.65 \\
\hline 80.0 & 75.0 & 73.3 & 80.1 & .8272 & .01984 & 40.97 & .0889 & 15.63 \\
\hline 80.0 & 74.0 & 71.9 & 76.4 & .7888 & .01889 & 39.93 & .0870 & 15.60 \\
\hline 80.0 & 73.0 & 70.5 & 72.8 & .7513 & .01797 & 38.92 & .0851 & $\begin{array}{l}10.00 \\
15.58\end{array}$ \\
\hline 80.0 & 72.0 & 69.0 & 69.2 & .7145 & .01706 & 37.93 & .0832 & 15.56 \\
\hline 80.0 & 71.0 & 67.5 & 65.7 & .6785 & .01618 & 36.96 & .0814 & 15.54 \\
\hline 80.0 & 70.0 & 66.0 & 62.3 & 6433 & .01532 & 36.02 & .0796 & 15.52 \\
\hline 80.0 & 69.0 & 64.4 & 59.0 & .6089 & .01448 & 35.10 & .0778 & 15.50 \\
\hline 80.0 & 68.0 & 62.8 & 55.7 & .5752 & .01366 & 34.20 & .0761 & 15.48 \\
\hline 80.0 & 67.0 & 61.1 & 52.5 & .5422 & .01286 & 33.33 & .0744 & 15.46 \\
\hline 80.0 & 66.0 & 59.4 & 49.4 & .5099 & .01208 & 32.47 & .0728 & 15.44 \\
\hline 80.0 & 65.0 & 57.6 & 46.3 & .4782 & .01132 & 31.63 & .0712 & 15.42 \\
\hline 80.0 & 64.0 & 55.7 & 43.3 & .4473 & .01057 & 30.82 & .0696 & 15.40 \\
\hline 80.0 & 63.0 & 53.8 & 40.4 & .4170 & .00984 & 30.02 & .0680 & 15.38 \\
\hline 80.0 & 62.0 & 51.8 & 37.5 & .3873 & .00913 & 29.24 & .0665 & 15.37 \\
\hline 80.0 & 61.0 & 49.7 & 34.7 & .3582 & .00844 & 28.48 & .0650 & 15.35 \\
\hline 80.0 & 60.0 & 47.5 & 31.9 & .3297 & .00776 & 27.73 & .0636 & 15.33 \\
\hline 80.0 & 59.0 & 45.1 & 29.2 & .3019 & .00709 & 27.01 & .0621 & 15.32 \\
\hline 80.0 & 58.0 & 42.7 & 26.6 & .2745 & .00645 & 26.30 & .0607 & 15.30 \\
\hline 80.0 & 57.0 & 40.0 & 24.0 & .2478 & .00581 & 25.60 & .0593 & 15.29 \\
\hline 80.0 & 56.0 & 37.2 & 21.5 & .2216 & .00519 & 24.92 & .0580 & 15.27 \\
\hline 80.0 & 55.0 & 34.1 & 19.0 & .1959 & .00459 & 24.26 & .0566 & 15.26 \\
\hline 80.0 & 54.0 & 30.8 & 16.5 & .1707 & .00399 & 23.61 & .0553 & 15.24 \\
\hline 80.0 & 53.0 & 27.4 & 14.1 & .1460 & .00341 & 22.97 & .0540 & 15.23 \\
\hline 80.0 & 52.0 & 23.6 & 11.8 & .1218 & .00284 & 22.35 & .0527 & 15.21 \\
\hline 80.0 & 51.0 & 19.0 & 9.5 & .0981 & .00229 & 21.74 & .0514 & 15.20 \\
\hline 80.0 & 50.0 & 13.5 & 7.3 & .0749 & .00174 & 21.14 & .0502 & 15.19 \\
\hline 80.0 & 49.0 & 6.3 & 5.0 & .0521 & .00121 & 20.56 & .0489 & 15.18 \\
\hline 80.0 & 48.0 & -4.5 & 2.9 & .0297 & .00069 & 19.99 & .0477 & 15.16 \\
\hline 80.0 & 47.0 & -28.3 & .8 & .0078 & .00018 & 19.43 & .0464 & 15.15 \\
\hline
\end{tabular}


$\mathrm{PB}=26.87$, Altitude $=3000-$ Continued

\begin{tabular}{|c|c|c|c|c|c|c|c|c|}
\hline $\mathrm{DB}$ & WB & $\mathrm{DP}$ & $\mathrm{RH}$ & PV & $\mathrm{W}$ & $\mathrm{H}$ & S & V \\
\hline 120.0 & 120.0 & 120.0 & 100.0 & 3.4476 & .09209 & 131.35 & .2506 & 18.67 \\
\hline 120.0 & 118.0 & 117.8 & 94.0 & 3.2425 & .08585 & 124.41 & .2385 & 18.51 \\
\hline 120.0 & 116.0 & 115.6 & 88.3 & 3.0459 & .07997 & 117.87 & .2271 & 18.35 \\
\hline 120.0 & 114.0 & 113.3 & 82.9 & 2.8576 & .07443 & 111.71 & .2163 & 18.21 \\
\hline 120.0 & 112.0 & 111.1 & 77.7 & 2.6773 & .06921 & 105.89 & .2061 & 18.07 \\
\hline 120.0 & 110.0 & 108.8 & 72.6 & 2.5046 & .06428 & 100.41 & .1964 & 17.95 \\
\hline 120.0 & 108.0 & 106.4 & 67.9 & 2.3393 & .05963 & 95.24 & . 1872 & 7.82 \\
\hline 120.0 & 106.0 & 104.0 & 63.3 & 2.1811 & .05523 & 90.35 & . 1785 & 7.71 \\
\hline 120.0 & 104.0 & 101.6 & 58.9 & 2.0297 & & 85.72 & . 1703 & .60 \\
\hline 120.0 & 102.0 & 99.2 & 54.7 & 1.8848 & .04715 & 81.35 & .1624 & 17.50 \\
\hline 120.0 & 100.0 & 96.6 & 50.6 & 1.7461 & .04344 & 77.22 & .1550 & 17.40 \\
\hline 120.0 & 98.0 & 94.1 & 46.8 & 1.6135 & .03992 & 73.31 & .1479 & 7.31 \\
\hline 120.0 & 96.0 & 91.4 & 43.1 & 1.4866 & .03660 & 69.61 & .1412 & 7.23 \\
\hline 120.0 & 94.0 & 88.7 & 39.6 & 1.3652 & .03345 & 66.10 & .1348 & 17.14 \\
\hline 120.0 & 92.0 & 85.9 & 36.2 & 1.2490 & .03046 & 62.77 & .1287 & 17.07 \\
\hline 120.0 & 90.0 & 83.0 & 33.0 & 1.1380 & .02763 & 59.62 & .1229 & 16.99 \\
\hline 120.0 & 88.0 & 80.0 & 29.9 & 1.0317 & .02495 & 56.63 & .1174 & 16.92 \\
\hline 120.0 & 86.0 & 76.8 & 27.0 & .9301 & .02240 & 53.80 & .1121 & 16.86 \\
\hline 120.0 & 84.0 & 73.5 & 24.2 & .8330 & .01998 & 51.11 & .1071 & 6.79 \\
\hline 120.0 & 82.0 & 70.0 & 21.5 & .7400 & .01769 & 48.55 & (1023 & 16.73 \\
\hline 120.0 & 80.0 & 66.3 & 18.9 & .6511 & .01551 & 46.13 & .0977 & 16.68 \\
\hline 120.0 & 78.0 & 62.3 & 16.4 & .5661 & .01344 & 43.82 & .0933 & 16.62 \\
\hline 120.0 & 76.0 & 57.9 & 14.1 & .4847 & .01147 & 41.63 & .0891 & 16.57 \\
\hline 120.0 & 74.0 & 53.1 & 11.8 & .4068 & .00960 & 39.54 & .0850 & 5.52 \\
\hline 120.0 & 72.0 & 47.7 & 9.6 & .3323 & .00782 & 37.56 & .0811 & 47 \\
\hline 120.0 & 70.0 & 41.3 & 7.6 & .2610 & .00612 & 35.67 & .0774 & 16.43 \\
\hline 120.0 & 68.0 & 33.6 & 5.6 & .1927 & .00451 & 33.87 & .0738 & 6.39 \\
\hline 120.0 & 66.0 & 24.5 & 3.7 & .1273 & .00297 & 32.16 & .0703 & 16.35 \\
\hline 120.0 & 64.0 & 10.6 & 1.9 & .0647 & .00151 & 30.53 & .0669 & \\
\hline 120.0 & 62.0 & -36.6 & .1 & .0047 & .00011 & 28.97 & .0634 & 16.27 \\
\hline
\end{tabular}


$\mathrm{PB}=26.87$, Altitude $=3000-$ Continued

\begin{tabular}{|c|c|c|c|c|c|c|c|c|}
\hline $\mathrm{DB}$ & WB & $\mathrm{DP}$ & $\mathrm{RH}$ & PV & W & $\mathrm{H}$ & S & V \\
\hline 160.0 & 160.0 & 160.0 & 100.0 & 9.6555 & .35199 & 436.12 & .7602 & 27.16 \\
\hline 160.0 & 158.0 & 157.9 & 95.2 & 9.1896 & .32611 & 406.92 & .7124 & 26.44 \\
\hline 160.0 & 156.0 & 155.9 & 90.5 & 8.7411 & .30246 & 380.24 & .6685 & 25.79 \\
\hline 160.0 & 154.0 & 153.8 & 86.1 & 8.3094 & .28078 & 355.78 & .6282 & 25.19 \\
\hline 160.0 & 152.0 & 151.7 & 81.8 & 7.8942 & .26086 & 333.30 & .5911 & 24.64 \\
\hline 160.0 & 150.0 & 149.6 & 77.6 & 7.4947 & .24251 & 312.59 & .5567 & 24.13 \\
\hline 160.0 & 148.0 & 147.5 & 73.6 & 7.1106 & .22557 & 293.46 & .5249 & 23.66 \\
\hline 160.0 & 146.0 & 145.3 & 69.8 & 6.7414 & .20990 & 275.76 & .4954 & 23.23 \\
\hline 160.0 & 144.0 & 143.2 & 66.1 & 6.3866 & .19537 & 259.36 & .4680 & 22.83 \\
\hline 160.0 & 142.0 & 141.1 & 62.6 & 6.0456 & .18189 & 244.13 & .4424 & 22.45 \\
\hline 160.0 & 140.0 & 138.9 & 59.2 & 5.7181 & .16934 & 229.96 & .4185 & 22.11 \\
\hline 160.0 & 138.0 & 136.7 & 56.0 & 5.4036 & .15766 & 216.76 & .3963 & 21.78 \\
\hline 160.0 & 136.0 & 134.5 & 52.8 & 5.1016 & .14676 & 204.45 & .3754 & 21.48 \\
\hline 160.0 & 134.0 & 132.3 & 49.8 & 4.8118 & .13658 & 192.95 & .3558 & 21.20 \\
\hline 160.0 & 132.0 & 130.1 & 47.0 & 4.5337 & 12707 & 182.20 & .3375 & 20.93 \\
\hline 160.0 & 130.0 & 127.8 & 44.2 & 4.2668 & .11816 & 172.13 & .3203 & 20.69 \\
\hline 160.0 & 128.0 & 125.5 & 41.5 & 4.0109 & .10982 & 162.70 & .3041 & 20.45 \\
\hline 160.0 & 126.0 & 123.2 & 39.0 & 3.7655 & 10199. & 153.85 & .2888 & 20.24 \\
\hline 160.0 & 124.0 & 120.9 & 36.6 & 3.5302 & .09464 & 145.54 & .2744 & 20.03 \\
\hline 160.0 & 122.0 & 118.5 & 34.2 & 3.3047 & .08773 & 137.73 & .2609 & 19.84 \\
\hline 160.0 & 120.0 & 116.1 & 32.0 & 3.0886 & .08124 & 130.39 & 2481 & 19.66 \\
\hline 160.0 & 118.0 & 113.6 & 29.8 & 2.8815 & .07513 & 123.48 & .2360 & 19.49 \\
\hline 160.0 & 116.0 & 111.1 & 27.8 & 2.6832 & .06938 & 116.98 & .2246 & 19.33 \\
\hline 160.0 & 114.0 & 108.6 & 25.8 & 2.4933 & .06396 & 110.85 & .2138 & 19.18 \\
\hline 160.0 & 112.0 & 106.0 & 23.9 & 2.3114 & .05885 & 105.07 & .2035 & 19.04 \\
\hline 160.0 & 110.0 & 103.4 & 22.1 & 2.1373 & .05402 & 99.61 & 1938 & 18.90 \\
\hline 160.0 & 108.0 & 100.6 & 20.4 & 1.9706 & .04947 & 94.46 & .1846 & 18.78 \\
\hline 160.0 & 106.0 & 97.8 & 18.8 & 1.8111 & .04518 & 89.60 & .1759 & 18.66 \\
\hline 160.0 & 104.0 & 95.0 & 17.2 & 1.6585 & .04111 & 85.01 & .1676 & 18.54 \\
\hline 160.0 & 102.0 & 92.0 & 15.7 & 1.5125 & .03727 & 80.66 & .1598 & 18.44 \\
\hline 160.0 & 100.0 & 88.9 & 14.2 & 1.3728 & .03364 & 76.55 & .1523 & 18.33 \\
\hline 160.0 & 98.0 & 85.7 & 12.8 & 1.2392 & .03021 & 72.67 & .1452 & 18.24 \\
\hline 160.0 & 96.0 & 82.3 & 11.5 & 1.1114 & .02696 & 68.99 & .1384 & 18.15 \\
\hline 160.0 & 94.0 & 78.7 & 10.2 & .9892 & .02388 & 65.50 & .1320 & 18.06 \\
\hline 160.0 & 92.0 & 74.9 & 9.0 & .8724 & .02096 & 62.20 & .1258 & 17.98 \\
\hline 160.0 & 90.0 & 70.8 & 7.9 & .7606 & .01820 & 59.07 & .1199 & 17.90 \\
\hline 160.0 & 88.0 & 66.4 & 6.8 & .6538 & .01558 & 56.11 & .1143 & 17.83 \\
\hline 160.0 & 86.0 & 61.6 & 5.7 & .5517 & .01309. & 53.29 & .1090 & 17.76 \\
\hline 160.0 & 84.0 & 56.1 & 4.7 & .4540 & .01073. & 50.63 & .1038 & 17.70 \\
\hline 160.0 & 82.0 & 49.9 & 3.7 & .3606 & .00849 & 48.09 & .0989 & 17.63 \\
\hline 160.0 & 80.0 & 42.4 & 2.8 & .2713 & .00637 & 45.69 & .0942 & 17.57 \\
\hline 160.0 & 78.0 & 32.8 & 1.9 & .1859 & .00435 & 43.40 & .0896 & 17.52 \\
\hline 160.0 & 76.0 & 20.3 & 1.1 & .1042 & .00243 & 41.23 & .0852 & 17.46 \\
\hline 160.0 & 74.0 & -6.9 & .3 & .0261 & .00061 & 39.16 & .0808 & 17.41 \\
\hline
\end{tabular}


$\mathrm{PB}=25.88$, Altitude $=4000$

\begin{tabular}{|c|c|c|c|c|c|c|c|c|}
\hline DB & WB & $\mathrm{DP}$ & $\mathrm{RH}$ & PV & W & $\mathrm{H}$ & S & V \\
\hline-40.0 & -40.0 & -40.0 & 100.0 & .0038 & .00009 & -9.49 & -.0116 & 12.22 \\
\hline $\begin{array}{l}.0 \\
.0 \\
.0 \\
.0 \\
.0\end{array}$ & $\begin{array}{r}.0 \\
-.1 \\
-.2 \\
-.3 \\
-.4\end{array}$ & $\begin{array}{r}.0 \\
-.5 \\
-1.1 \\
-1.6 \\
-2.2\end{array}$ & $\begin{array}{r}100.0 \\
97.3 \\
94.6 \\
91.9 \\
89.3\end{array}$ & $\begin{array}{l}.0376 \\
.0366 \\
.0356 \\
.0346 \\
.0336\end{array}$ & $\begin{array}{l}.00091 \\
.00089 \\
.00086 \\
.00084 \\
.00081\end{array}$ & $\begin{array}{l}.98 \\
.96 \\
.93 \\
.90 \\
.88\end{array}$ & $\begin{array}{l}.0122 \\
.0121 \\
.0121 \\
.0120 \\
.0120\end{array}$ & $\begin{array}{l}13.41 \\
13.41 \\
13.41 \\
13.41 \\
13.41\end{array}$ \\
\hline $\begin{array}{l}.0 \\
.0 \\
.0 \\
.0 \\
.0\end{array}$ & $\begin{array}{l}-.5 \\
-.6 \\
-.7 \\
-.8 \\
-.9\end{array}$ & $\begin{array}{l}-2.7 \\
-3.3 \\
-3.9 \\
-4.6 \\
-5.2\end{array}$ & $\begin{array}{l}86.6 \\
83.9 \\
81.2 \\
78.6 \\
75.9\end{array}$ & $\begin{array}{l}.0326 \\
.0316 \\
.0306 \\
.0296 \\
.0286\end{array}$ & $\begin{array}{l}.00079 \\
.00076 \\
.00074 \\
.00071 \\
.00069\end{array}$ & $\begin{array}{l}.85 \\
.83 \\
.80 \\
.77 \\
.75\end{array}$ & $\begin{array}{l}.0119 \\
.0119 \\
.0118 \\
.0117 \\
.0117\end{array}$ & $\begin{array}{l}13.40 \\
13.40 \\
13.40 \\
13.40 \\
13.40\end{array}$ \\
\hline $\begin{array}{l}.0 \\
.0 \\
.0 \\
.0 \\
.0\end{array}$ & $\begin{array}{l}-1.0 \\
-1.1 \\
-1.2 \\
-1.3 \\
-1.4\end{array}$ & $\begin{array}{l}-5.9 \\
-6.6 \\
-7.3 \\
-8.0 \\
-8.8\end{array}$ & $\begin{array}{l}73.2 \\
70.6 \\
67.9 \\
65.2 \\
62.6\end{array}$ & $\begin{array}{l}.0276 \\
.0266 \\
.0256 \\
.0246 \\
.0236\end{array}$ & $\begin{array}{l}.00067 \\
.00064 \\
.00062 \\
.00059 \\
.00057\end{array}$ & $\begin{array}{l}.72 \\
.70 \\
.67 \\
.65 \\
.62\end{array}$ & $\begin{array}{l}.0116 \\
.0116 \\
.0115 \\
.0115 \\
.0114\end{array}$ & $\begin{array}{l}13.40 \\
13.40 \\
13.40 \\
13.40 \\
13.40\end{array}$ \\
\hline $\begin{array}{l}.0 \\
.0 \\
.0 \\
.0 \\
.0\end{array}$ & $\begin{array}{l}-1.5 \\
-1.6 \\
-1.7 \\
-1.8 \\
-1.9\end{array}$ & $\begin{array}{l}-9.6 \\
-10.4 \\
-11.3 \\
-12.2 \\
-13.1\end{array}$ & $\begin{array}{l}59.9 \\
57.3 \\
54.6 \\
52.0 \\
49.3\end{array}$ & $\begin{array}{l}.0226 \\
.0216 \\
.0206 \\
.0196 \\
.0186\end{array}$ & $\begin{array}{l}.00054 \\
.00052 \\
.00050 \\
.00047 \\
.00045\end{array}$ & $\begin{array}{l}.59 \\
.57 \\
.54 \\
.52 \\
.49\end{array}$ & $\begin{array}{l}.0113 \\
.0113 \\
.0112 \\
.0112 \\
.0111\end{array}$ & $\begin{array}{l}13.40 \\
13.40 \\
13.40 \\
13.40 \\
13.40\end{array}$ \\
\hline $\begin{array}{l}.0 \\
.0 \\
.0 \\
.0 \\
.0\end{array}$ & $\begin{array}{l}-2.0 \\
-2.1 \\
-2.2 \\
-2.3 \\
-2.4\end{array}$ & $\begin{array}{l}-14.1 \\
-15.1 \\
-16.2 \\
-17.4 \\
-18.6\end{array}$ & $\begin{array}{l}46.7 \\
44.1 \\
41.4 \\
38.8 \\
36.2\end{array}$ & $\begin{array}{l}.0176 \\
.0166 \\
.0156 \\
.0146 \\
.0136\end{array}$ & $\begin{array}{l}.00042 \\
.00040 \\
.00038 \\
.00035 \\
.00033\end{array}$ & $\begin{array}{l}.47 \\
.44 \\
.42 \\
.39 \\
.37\end{array}$ & $\begin{array}{l}.0111 \\
.0110 \\
.0109 \\
.0109 \\
.0108\end{array}$ & $\begin{array}{l}13.40 \\
13.40 \\
13.40 \\
13.40 \\
13.40\end{array}$ \\
\hline $\begin{array}{l}.0 \\
.0 \\
.0 \\
.0 \\
.0 \\
.0\end{array}$ & $\begin{array}{l}-2.5 \\
-2.6 \\
-2.7 \\
-2.8 \\
-2.9 \\
-3.0\end{array}$ & $\begin{array}{l}-19.9 \\
-21.4 \\
-22.9 \\
-24.6 \\
-26.4 \\
-28.4\end{array}$ & $\begin{array}{l}33.5 \\
30.9 \\
28.3 \\
25.7 \\
23.0 \\
20.4\end{array}$ & $\begin{array}{l}.0126 \\
.0116 \\
.0106 \\
.0097 \\
.0087 \\
.0077\end{array}$ & $\begin{array}{l}.00030 \\
.00028 \\
.00026 \\
.00023 \\
.00021 \\
.00019\end{array}$ & $\begin{array}{l}.34 \\
.31 \\
.29 \\
.26 \\
.24 \\
.21\end{array}$ & $\begin{array}{l}.0108 \\
.0107 \\
.0106 \\
.0106 \\
.0105 \\
.0105\end{array}$ & $\begin{array}{l}13.39 \\
13.39 \\
13.39 \\
13.39 \\
13.39 \\
13.39\end{array}$ \\
\hline $\begin{array}{l}40.0 \\
40.0 \\
40.0 \\
40.0 \\
40.0\end{array}$ & $\begin{array}{l}40.0 \\
39.0 \\
38.0 \\
37.0 \\
36.0\end{array}$ & $\begin{array}{l}40.0 \\
38.0 \\
35.9 \\
33.6 \\
31.3\end{array}$ & $\begin{array}{r}100.0 \\
92.4 \\
85.0 \\
77.7 \\
70.6\end{array}$ & $\begin{array}{l}.2477 \\
.2290 \\
.2106 \\
.1925 \\
.1748\end{array}$ & $\begin{array}{l}.00603 \\
.00557 \\
.00512 \\
.00468 \\
.00425\end{array}$ & $\begin{array}{l}16.13 \\
15.63 \\
15.15 \\
14.67 \\
14.20\end{array}$ & $\begin{array}{l}.0437 \\
.0427 \\
.0417 \\
.0408 \\
.0398\end{array}$ & $\begin{array}{l}14.70 \\
14.69 \\
14.68 \\
14.67 \\
14.66\end{array}$ \\
\hline $\begin{array}{l}40.0 \\
40.0 \\
40.0 \\
40.0 \\
40.0\end{array}$ & $\begin{array}{l}35.0 \\
34.0 \\
33.0 \\
32.0 \\
31.0\end{array}$ & $\begin{array}{l}29.0 \\
26.6 \\
23.8 \\
22.4 \\
19.3\end{array}$ & $\begin{array}{l}63.5 \\
56.6 \\
49.8 \\
46.6 \\
40.0\end{array}$ & $\begin{array}{l}.1573 \\
.1402 \\
.1233 \\
.1154 \\
.0992\end{array}$ & $\begin{array}{l}.00382 \\
.00340 \\
.00299 \\
.00280 \\
.00240\end{array}$ & $\begin{array}{l}13.74 \\
13.29 \\
12.85 \\
12.64 \\
12.21\end{array}$ & $\begin{array}{l}.0389 \\
.0379 \\
.0370 \\
.0366 \\
.0357\end{array}$ & $\begin{array}{l}14.65 \\
14.64 \\
14.63 \\
14.62 \\
14.61\end{array}$ \\
\hline $\begin{array}{l}40.0 \\
40.0 \\
40.0 \\
40.0 \\
40.0 \\
40.0\end{array}$ & $\begin{array}{l}30.0 \\
29.0 \\
28.0 \\
27.0 \\
26.0 \\
25.0\end{array}$ & $\begin{array}{r}15.7 \\
11.5 \\
6.4 \\
-.1 \\
-9.4 \\
-27.0\end{array}$ & \begin{tabular}{r|}
33.6 \\
27.3 \\
21.2 \\
15.1 \\
9.2 \\
3.4
\end{tabular} & $\begin{array}{l}.0833 \\
.0677 \\
.0525 \\
.0375 \\
.0228 \\
.0084\end{array}$ & $\begin{array}{l}.00202 \\
.00164 \\
.00127 \\
.00091 \\
.00055 \\
.00020\end{array}$ & $\begin{array}{r}11.80 \\
11.39 \\
10.99 \\
10.60 \\
10.22 \\
9.84\end{array}$ & $\begin{array}{l}.0348 \\
.0340 \\
.0331 \\
.0323 \\
.0314 \\
.0306\end{array}$ & $\begin{array}{l}14.60 \\
14.60 \\
14.59 \\
14.58 \\
14.57 \\
14.56\end{array}$ \\
\hline
\end{tabular}


PB $=25.88$, Altitude $=4000-$ Continued

\begin{tabular}{|c|c|c|c|c|c|c|c|c|}
\hline $\mathrm{DB}$ & WB & $\mathrm{DP}$ & $\mathrm{RH}$ & PV & W & $\mathrm{H}$ & $\mathrm{S}$ & $\mathrm{V}$ \\
\hline $\begin{array}{l}80.0 \\
80.0 \\
80.0 \\
80.0 \\
80.0\end{array}$ & $\begin{array}{l}80.0 \\
79.0 \\
78.0 \\
77.0 \\
76.0\end{array}$ & $\begin{array}{l}80.0 \\
78.7 \\
77.4 \\
76.1 \\
74.8\end{array}$ & $\begin{array}{r}100.0 \\
95.9 \\
91.9 \\
87.9 \\
84.1\end{array}$ & $\begin{array}{r}1.0323 \\
.9898 \\
.9483 \\
.9076 \\
.8679\end{array}$ & $\begin{array}{l}.02595 \\
.02484 \\
.02376 \\
.02270 \\
.02167\end{array}$ & $\begin{array}{l}47.67 \\
46.45 \\
45.27 \\
44.11 \\
42.98\end{array}$ & $\begin{array}{l}.1041 \\
.1019 \\
.0996 \\
.0975 \\
.0954\end{array}$ & $\begin{array}{l}16.38 \\
16.35 \\
16.32 \\
16.30 \\
16.27\end{array}$ \\
\hline $\begin{array}{l}80.0 \\
80.0 \\
80.0 \\
80.0 \\
80.0\end{array}$ & $\begin{array}{l}75.0 \\
74.0 \\
73.0 \\
72.0 \\
71.0\end{array}$ & $\begin{array}{l}73.4 \\
72.0 \\
70.6 \\
69.1 \\
67.6\end{array}$ & $\begin{array}{l}80.3 \\
76.6 \\
73.0 \\
69.5 \\
66.0\end{array}$ & $\begin{array}{r}.8290 \\
.7910 \\
.7538 \\
.7174 \\
.6818\end{array}$ & $\begin{array}{l}.02067 \\
.01969 \\
.01874 \\
.01781 \\
.01690\end{array}$ & $\begin{array}{l}41.88 \\
40.81 \\
39.77 \\
38.75 \\
37.75\end{array}$ & $\begin{array}{l}.0933 \\
.0913 \\
.0893 \\
.0874 \\
.0855\end{array}$ & $\begin{array}{l}16.25 \\
16.22 \\
16.20 \\
16.17 \\
16.15\end{array}$ \\
\hline $\begin{array}{l}80.0 \\
80.0 \\
80.0 \\
80.0 \\
80.0\end{array}$ & $\begin{array}{l}70.0 \\
69.0 \\
68.0 \\
67.0 \\
66.0\end{array}$ & $\begin{array}{l}66.1 \\
64.6 \\
63.0 \\
61.3 \\
59.6\end{array}$ & $\begin{array}{l}62.7 \\
59.4 \\
56.1 \\
53.0 \\
49.9\end{array}$ & $\begin{array}{l}.6469 \\
.6129 \\
.5795 \\
.5469 \\
.5149\end{array}$ & $\begin{array}{l}.01601 \\
.01515 \\
.01430 \\
.01348 \\
.01268\end{array}$ & $\begin{array}{l}36.78 \\
35.83 \\
34.91 \\
34.01 \\
33.13\end{array}$ & $\begin{array}{l}.0837 \\
.0819 \\
.0801 \\
.0784 \\
.0767\end{array}$ & $\begin{array}{l}16.13 \\
16.11 \\
16.09 \\
16.07 \\
16.04\end{array}$ \\
\hline $\begin{array}{l}80.0 \\
80.0 \\
80.0 \\
80.0 \\
80.0\end{array}$ & $\begin{array}{l}65.0 \\
64.0 \\
63.0 \\
62.0 \\
61.0\end{array}$ & $\begin{array}{l}57.9 \\
56.1 \\
54.2 \\
52.2 \\
50.2\end{array}$ & $\begin{array}{l}46.9 \\
43.9 \\
41.0 \\
38.1 \\
35.4\end{array}$ & $\begin{array}{l}.4836 \\
.4530 \\
.4231 \\
.3937 \\
.3650\end{array}$ & $\begin{array}{l}.01189 \\
.01113 \\
.01038 \\
.00965 \\
.00893\end{array}$ & $\begin{array}{l}32.27 \\
31.43 \\
30.61 \\
29.81 \\
29.02\end{array}$ & $\begin{array}{l}.0750 \\
.0734 \\
.0718 \\
.0702 \\
.0687\end{array}$ & $\begin{array}{l}16.03 \\
16.01 \\
15.99 \\
15.97 \\
15.95\end{array}$ \\
\hline $\begin{array}{l}80.0 \\
80.0 \\
80.0 \\
80.0 \\
80.0\end{array}$ & $\begin{array}{l}60.0 \\
59.0 \\
58.0 \\
57.0 \\
56.0\end{array}$ & $\begin{array}{l}48.1 \\
45.8 \\
43.4 \\
40.9 \\
38.1\end{array}$ & $\begin{array}{l}32.6 \\
30.0 \\
27.4 \\
24.8 \\
22.3\end{array}$ & $\begin{array}{l}.3369 \\
.3094 \\
.2824 \\
.2560 \\
.2302\end{array}$ & $\begin{array}{l}.00824 \\
.00755 \\
.00689 \\
.00624 \\
.00560\end{array}$ & $\begin{array}{l}28.26 \\
27.51 \\
26.78 \\
26.07 \\
25.37\end{array}$ & $\begin{array}{l}.0672 \\
.0657 \\
.0643 \\
.0629 \\
.0615\end{array}$ & $\begin{array}{l}15.93 \\
15.92 \\
15.90 \\
15.88 \\
15.87\end{array}$ \\
\hline $\begin{array}{l}80.0 \\
80.0 \\
80.0 \\
80.0 \\
80.0\end{array}$ & $\begin{array}{l}55.0 \\
54.0 \\
53.0 \\
52.0 \\
51.0\end{array}$ & $\begin{array}{l}35.2 \\
32.0 \\
28.8 \\
25.2 \\
21.1\end{array}$ & $\begin{array}{l}19.8 \\
17.4 \\
15.1 \\
12.8 \\
10.5\end{array}$ & $\begin{array}{l}.2048 \\
.1800 \\
.1557 \\
.1318 \\
.1085\end{array}$ & $\begin{array}{l}.00498 \\
.00437 \\
.00378 \\
.00320 \\
.00263\end{array}$ & $\begin{array}{l}24.69 \\
24.03 \\
23.37 \\
22.74 \\
22.11\end{array}$ & $\begin{array}{l}.0601 \\
.0587 \\
.0574 \\
.0561 \\
.0548\end{array}$ & $\begin{array}{l}15.85 \\
15.84 \\
15.82 \\
15.81 \\
15.79\end{array}$ \\
\hline $\begin{array}{l}80.0 \\
80.0 \\
80.0 \\
80.0\end{array}$ & $\begin{array}{l}50.0 \\
49.0 \\
48.0 \\
47.0\end{array}$ & $\begin{array}{r}16.2 \\
10.1 \\
1.7 \\
-12.2\end{array}$ & $\begin{array}{l}8.3 \\
6.1 \\
4.0 \\
1.9\end{array}$ & $\begin{array}{l}.0856 \\
.0631 \\
.0411 \\
.0195\end{array}$ & $\begin{array}{l}.00207 \\
.00153 \\
.00099 \\
.00047\end{array}$ & $\begin{array}{l}21.50 \\
20.91 \\
20.32 \\
19.75\end{array}$ & $\begin{array}{l}.0535 \\
.0523 \\
.0510 \\
.0497\end{array}$ & $\begin{array}{l}15.78 \\
15.76 \\
15.75 \\
15.74\end{array}$ \\
\hline
\end{tabular}


$\mathrm{PB}=25.88$, Altitude $=4000-$ Continued

\begin{tabular}{|c|c|c|c|c|c|c|c|c|}
\hline $\mathrm{DB}$ & WB & $\mathrm{DP}$ & $\mathrm{RH}$ & PV & W & $\mathrm{H}$ & $\mathrm{S}$ & V \\
\hline 120.0 & 120.0 & 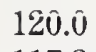 & 100.0 & 3.4476 & .09615 & 135.86 & .2614 & 19.49 \\
\hline 120.0 & 118.0 & 117.8 & 94.1 & 3.2432 & .08962 & 128.60 & .2487 & 19.32 \\
\hline 120.0 & 116.0 & 115.6 & 88.4 & 3.0474 & .08348 & 121.77 & .2368 & 19.15 \\
\hline 120.0 & 114.0 & 113.4 & 83.0 & 2.8598 & .07769 & 115.34 & .2255 & 18.99 \\
\hline 120.0 & 112.0 & 111.1 & 77.7 & 2.6803 & .07224 & 109.28 & .2149 & 18.85 \\
\hline 120.0 & 110.0 & 108.8 & 72.8 & 2.5083 & .06711 & 103.56 & .2048 & 18.71 \\
\hline 120.0 & 108.0 & 106.5 & 68.0 & 2.3438 & .06226 & 98.17 & .1953 & 18.58 \\
\hline 120.0 & 106.0 & 104.1 & 63.4 & 2.1863 & .05768 & 93.08 & .1862 & 18.45 \\
\hline 120.0 & 104.0 & 101.7 & 59.0 & 2.0356 & .05336 & 88.27 & .1776 & 18.34 \\
\hline 120.0 & 102.0 & 99.3 & 54.9 & 1.8914 & .04928 & 83.73 & .1695 & 18.23 \\
\hline 120.0 & 100.0 & 96.8 & 50.9 & 1.7535 & .04542 & 79.43 & .1617 & 18.12 \\
\hline 120.0 & 98.0 & 94.2 & 47.0 & 1.6215 & .04177 & 75.37 & .1544 & 18.02 \\
\hline 120.0 & 96.0 & 91.6 & 43.4 & 1.4953 & .03832 & 71.52 & .1474 & 17.93 \\
\hline 120.0 & 94.0 & 88.9 & 39.9 & 1.3747 & .03505 & 67.89 & .1408 & 17.84 \\
\hline 120.0 & 92.0 & 86.2 & 36.5 & 1.2593 & .03195 & 64.44 & .1345 & 17.76 \\
\hline 120.0 & 90.0 & 83.3 & 33.3 & 1.1489 & .02902 & 61.17 & .1285 & 17.68 \\
\hline 120.0 & 88.0 & 80.3 & 30.3 & 1.0434 & .02624 & 58.08 & .1227 & 17.60 \\
\hline 120.0 & 86.0 & 77.2 & 27.3 & .9425 & .02361 & 55.15 & .1173 & 17.53 \\
\hline 120.0 & 84.0 & 74.0 & 24.5 & .8461 & .02111 & 52.36 & .1121 & 17.47 \\
\hline 120.0 & 82.0 & 70.6 & 21.9 & .7538 & .01874 & 49.72 & .1071 & 17.40 \\
\hline 120.0 & 80.0 & 66.9 & 19.3 & .6656 & .01649 & 47.21 & .1024 & 17.34 \\
\hline 120.0 & 78.0 & 63.1 & 16.9 & .5813 & .01435 & 44.83 & .0978 & 17.28 \\
\hline 120.0 & 76.0 & 58.8 & 14.5 & .5006 & .01232 & 42.57 & .0935 & 17.23 \\
\hline 120.0 & 74.0 & 54.2 & 12.3 & .4234 & .01039 & 40.42 & .0893 & 17.17 \\
\hline 120.0 & 72.0 & 49.0 & 10.1 & .3496 & .00855 & 38.38 & .0853 & 17.13 \\
\hline 120.0 & 70.0 & 43.1 & 8.1 & .2790 & .00680 & 36.43 & .0815 & 17.08 \\
\hline 120.0 & 68.0 & 36.0 & 6.1 & .2114 & .00514 & 34.58 & .0778 & 17.03 \\
\hline 120.0 & 66.0 & 27.5 & 4.3 & .1467 & .00356 & 32.82 & .0742 & 16.99 \\
\hline 120.0 & 64.0 & 16.0 & 2.5 & .0848 & .00205 & 31.14 & .0707 & 16.95 \\
\hline 120.0 & 62.0 & -7.3 & .7 & .0255 & .00062 & 29.53 & .0673 & 16.91 \\
\hline
\end{tabular}


$\mathrm{PB}=25.88$, Altitude $=4000-$ Continued

\begin{tabular}{|c|c|c|c|c|c|c|c|c|}
\hline $\mathrm{DB}$ & WB & $\mathrm{DP}$ & $\mathrm{RH}$ & PV & W & $\mathrm{H}$ & S & $\mathrm{V}$ \\
\hline 160.0 & 160.0 & 160.0 & 100.0 & 9.6555 & .37343 & 460.35 & .8039 & 28.81 \\
\hline 160.0 & 158.0 & 157.9 & 95.2 & 9.1904 & .34547 & 428.80 & .7522 & 28.01 \\
\hline 160.0 & 156.0 & 155.9 & 90.5 & 8.7426 & 31999 & 400.05 & .7050 & 27.28 \\
\hline 160.0 & 154.0 & 153.8 & 86.1 & 8.3117 & .29670 & 373.77 & .6617 & 26.61 \\
\hline 160.0 & 152.0 & 151.7 & 81.8 & 7.8972 & .27535 & 349.68 & .6218 & 26.00 \\
\hline 160.0 & 150.0 & 149.6 & 77.7 & 7.4986 & .25574 & 327.53 & .5851 & 25.44 \\
\hline 160.0 & 148.0 & 147.5 & 73.7 & 7.1152 & .23766 & 307.13 & .5512 & 24.92 \\
\hline 160.0 & 146.0 & 145.4 & 69.9 & 6.7468 & .22098 & 288.29 & .5198 & 24.44 \\
\hline 160.0 & 144.0 & 143.2 & 66.2 & 6.3927 & .20554 & 270.86 & .4906 & 23.99 \\
\hline 160.0 & 142.0 & 141.1 & 62.7 & 6.0525 & .19123 & 254.69 & .4635 & 23.58 \\
\hline 160.0 & 140.0 & 138.9 & 59.3 & 5.7257 & .17794 & 239.69 & .4382 & 23.20 \\
\hline 160.0 & 138.0 & 136.8 & 56.0 & 5.4119 & .16559 & 225.73 & .4147 & 22.84 \\
\hline 160.0 & 136.0 & 134.6 & 52.9 & 5.1107 & .15408 & 212.73 & .3926 & 22.51 \\
\hline 160.0 & 134.0 & 132.4 & 49.9 & 4.8216 & .14335 & 200.60 & .3720 & 22.20 \\
\hline 160.0 & 132.0 & 130.1 & 47.1 & 4.5442 & .13332 & 189.27 & .3527 & 21.91 \\
\hline 160.0 & 130.0 & 127.9 & 44.3 & 4.2781 & .12395 & 178.68 & .3346 & 21.64 \\
\hline 160.0 & 128.0 & 125.6 & 41.7 & 4.0229 & .11518 & 168.77 & .3175 & 21.39 \\
\hline 160.0 & 126.0 & 123.3 & 39.1 & 3.7782 & .10696 & 159.48 & .3015 & 21.15 \\
\hline 160.0 & 124.0 & 121.0 & 36.7 & 3.5436 & .09926 & 150.77 & .2865 & 20.93 \\
\hline 160.0 & 122.0 & 118.6 & 34.4 & 3.3188 & .09202 & 142.59 & .2723 & 20.72 \\
\hline 160.0 & 120.0 & 116.2 & 32.1 & 3.1034 & .08523 & 134.90 & .2589 & 20.53 \\
\hline 160.0 & 118.0 & 113.8 & 30.0 & 2.8971 & .07884 & 127.68 & .2462 & 20.34 \\
\hline 160.0 & 116.0 & 111.3 & 28.0 & 2.6995 & .07282 & 120.88 & .2343 & 20.17 \\
\hline 160.0 & 114.0 & 108.8 & 26.0 & 2.5103 & .06716 & 114.48 & .2230 & 20.00 \\
\hline 160.0 & 112.0 & 106.3 & 24.1 & 2.3291 & .06183 & 108.44 & .2123 & 19.85 \\
\hline 160.0 & 110.0 & 103.6 & 22.3 & 2.1557 & .05680 & 102.76 & .2022 & 19.71 \\
\hline 160.0 & 108.0 & 101.0 & 20.6 & 1.9898 & .05206 & 97.39 & .1927 & 19.57 \\
\hline 160.0 & 106.0 & 98.2 & 19.0 & 1.8310 & .04758 & 92.33 & .1836 & 19.44 \\
\hline 160.0 & 104.0 & 95.4 & 17.4 & 1.6791 & .04336 & 87.55 & .1750 & 19.32 \\
\hline 160.0 & 102.0 & 92.4 & 15.9 & 1.5338 & .03937 & 83.03 & .1668 & 19.20 \\
\hline 160.0 & 100.0 & 89.4 & 14.4 & 1.3948 & .03559 & 78.76 & .1590 & 19.09 \\
\hline 160.0 & 98.0 & 86.2 & 13.1 & 1.2619 & .03202 & 74.72 & .1517 & 18.99 \\
\hline 160.0 & 96.0 & 82.9 & 11.8 & 1.1348 & .02865 & 70.90 & .1446 & 18.89 \\
\hline 160.0 & 94.0 & 79.4 & 10.5 & 1.0133 & .02545 & 67.29 & .1379 & 18.80 \\
\hline 160.0 & 92.0 & 75.7 & 9.3 & .8972 & .02243 & 63.87 & .1316 & 18.71 \\
\hline 160.0 & 90.0 & 71.8 & 8.1 & .7861 & .01957 & 60.63 & .1255 & 18.63 \\
\hline 160.0 & 88.0 & 67.6 & 7.0 & .6800 & .01685 & 57.55 & .1197 & 18.55 \\
\hline 160.0 & 86.0 & 62.9 & 6.0 & .5785 & .01428 & 54.64 & .1142 & 18.47 \\
\hline 160.0 & 84.0 & 57.8 & 5.0 & .4816 & .01184 & 51.88 & .1089 & 18.40 \\
\hline 160.0 & 82.0 & 51.9 & 4.0 & .3889 & .00953 & 49.26 & .1038 & 18.34 \\
\hline 160.0 & 80.0 & 45.0 & 3.1 & .3003 & .00733 & 46.77 & .0989 & 18.27 \\
\hline 160.0 & 78.0 & 36.5 & 2.2 & .2155 & .00524 & 44.41 & .0942 & 18.21 \\
\hline 160.0 & 76.0 & 25.7 & 1.4 & .1346 & .00326 & 42.17 & .0897 & 18.16 \\
\hline 160.0 & 74.0 & 8.1 & .6 & .0571 & .00138 & 40.04 & .0853 & 18.10 \\
\hline
\end{tabular}


$\mathrm{PB}=24.89$, Altitude $=5000$

\begin{tabular}{|c|c|c|c|c|c|c|c|c|}
\hline DB & WB & $\mathrm{DP}$ & $\mathrm{RH}$ & PV & W & $\mathrm{H}$ & S & V \\
\hline-40.0 & -40.0 & -40.0 & 100.0 & .0038 & .00010 & -9.48 & -.0089 & 12.71 \\
\hline $\begin{array}{l}.0 \\
.0 \\
.0 \\
.0 \\
.0\end{array}$ & $\begin{array}{r}.0 \\
-.1 \\
-.2 \\
-.3 \\
-.4\end{array}$ & $\begin{array}{r}.0 \\
-.5 \\
-1.0 \\
-1.5 \\
-2.1\end{array}$ & $\begin{array}{r}100.0 \\
97.4 \\
94.8 \\
92.2 \\
89.6\end{array}$ & $\begin{array}{l}.0376 \\
.0367 \\
.0357 \\
.0347 \\
.0337\end{array}$ & $\begin{array}{l}.00095 \\
.00092 \\
.00090 \\
.00087 \\
.00085\end{array}$ & $\begin{array}{r}1.02 \\
1.00 \\
.97 \\
.95 \\
.92\end{array}$ & $\begin{array}{l}.0150 \\
.0149 \\
.0149 \\
.0148 \\
.0147\end{array}$ & $\begin{array}{l}13.94 \\
13.94 \\
13.94 \\
13.94 \\
13.94\end{array}$ \\
\hline $\begin{array}{l}.0 \\
.0 \\
.0 \\
.0 \\
.0\end{array}$ & $\begin{array}{l}-.5 \\
-.6 \\
-.7 \\
-.8 \\
-.9\end{array}$ & $\begin{array}{l}-2.6 \\
-3.2 \\
-3.8 \\
-4.4 \\
-5.0\end{array}$ & $\begin{array}{l}87.0 \\
84.4 \\
81.8 \\
79.2 \\
76.6\end{array}$ & $\begin{array}{l}.0327 \\
.0318 \\
.0308 \\
.0298 \\
.0288\end{array}$ & $\begin{array}{l}.00082 \\
.00080 \\
.00077 \\
.00075 \\
.00072\end{array}$ & $\begin{array}{l}.89 \\
.87 \\
.84 \\
.82 \\
.79\end{array}$ & $\begin{array}{l}.0147 \\
.0146 \\
.0146 \\
.0145 \\
.0145\end{array}$ & $\begin{array}{l}13.94 \\
13.94 \\
13.94 \\
13.94 \\
13.94\end{array}$ \\
\hline $\begin{array}{l}.0 \\
.0 \\
.0 \\
.0 \\
.0\end{array}$ & $\begin{array}{r}-1.0 \\
-1.1 \\
-1.2 \\
-1.3 \\
-1.4\end{array}$ & $\begin{array}{l}-5.7 \\
-6.3 \\
-7.0 \\
-7.7 \\
-8.4\end{array}$ & $\begin{array}{l}74.1 \\
71.5 \\
68.9 \\
66.3 \\
63.7\end{array}$ & $\begin{array}{l}.0279 \\
.0269 \\
.0259 \\
.0250 \\
.0240\end{array}$ & $\begin{array}{l}.00070 \\
.00068 \\
.00065 \\
.00063 \\
.00060\end{array}$ & $\begin{array}{l}.76 \\
.74 \\
.71 \\
.69 \\
.66\end{array}$ & $\begin{array}{l}.0144 \\
.0143 \\
.0143 \\
.0142 \\
.0142\end{array}$ & $\begin{array}{l}13.94 \\
13.94 \\
13.94 \\
13.93 \\
13.93\end{array}$ \\
\hline $\begin{array}{l}.0 \\
.0 \\
.0 \\
.0 \\
.0\end{array}$ & $\begin{array}{l}-1.5 \\
-1.6 \\
-1.7 \\
-1.8 \\
-1.9\end{array}$ & $\begin{array}{r}-9.2 \\
-10.0 \\
-10.8 \\
-11.6 \\
-12.5\end{array}$ & $\begin{array}{l}61.2 \\
58.6 \\
56.0 \\
53.5 \\
50.9\end{array}$ & $\begin{array}{l}.0230 \\
.0221 \\
.0211 \\
.0201 \\
.0192\end{array}$ & $\begin{array}{l}.00058 \\
.00055 \\
.00053 \\
.00051 \\
.00048\end{array}$ & $\begin{array}{l}.63 \\
.61 \\
.58 \\
.56 \\
.53\end{array}$ & $\begin{array}{l}.0141 \\
.0140 \\
.0140 \\
.0139 \\
.0139\end{array}$ & $\begin{array}{l}13.93 \\
13.93 \\
13.93 \\
13.93 \\
13.93\end{array}$ \\
\hline $\begin{array}{l}.0 \\
.0 \\
.0 \\
.0 \\
.0\end{array}$ & $\begin{array}{l}-2.0 \\
-2.1 \\
-2.2 \\
-2.3 \\
-2.4\end{array}$ & $\begin{array}{l}-13.5 \\
-14.4 \\
-15.5 \\
-16.5 \\
-17.7\end{array}$ & $\begin{array}{l}48.4 \\
45.8 \\
43.2 \\
40.7 \\
38.1\end{array}$ & $\begin{array}{l}.0182 \\
.0172 \\
.0163 \\
.0153 \\
.0144\end{array}$ & $\begin{array}{l}.00046 \\
.00043 \\
.00041 \\
.00038 \\
.00036\end{array}$ & $\begin{array}{l}.51 \\
.48 \\
.45 \\
.43 \\
.40\end{array}$ & $\begin{array}{l}.0138 \\
.0138 \\
.0137 \\
.0136 \\
.0136\end{array}$ & $\begin{array}{l}13.93 \\
13.93 \\
13.93 \\
13.93 \\
13.93\end{array}$ \\
\hline $\begin{array}{l}.0 \\
.0 \\
.0 \\
.0 \\
.0\end{array}$ & $\begin{array}{l}-2.5 \\
-2.6 \\
-2.7 \\
-2.8 \\
-2.9\end{array}$ & $\begin{array}{l}-18.9 \\
-20.2 \\
-21.6 \\
-23.1 \\
-24.7\end{array}$ & $\begin{array}{l}35.6 \\
33.1 \\
30.5 \\
28.0 \\
25.4\end{array}$ & $\begin{array}{l}.0134 \\
.0124 \\
.0115 \\
.0105 \\
.0096\end{array}$ & $\begin{array}{l}.00034 \\
.00031 \\
.00029 \\
.00026 \\
.00024\end{array}$ & $\begin{array}{l}.38 \\
.35 \\
.33 \\
.30 \\
.28\end{array}$ & $\begin{array}{l}.0135 \\
.0135 \\
.0134 \\
.0133 \\
.0133\end{array}$ & $\begin{array}{l}13.93 \\
13.93 \\
13.93 \\
13.93 \\
13.93\end{array}$ \\
\hline $\begin{array}{l}.0 \\
.0\end{array}$ & $\begin{array}{l}-3.0 \\
-3.1\end{array}$ & $\begin{array}{l}-26.5 \\
-28.5\end{array}$ & $\begin{array}{l}22.9 \\
20.4\end{array}$ & $\begin{array}{l}.0086 \\
.0077\end{array}$ & $\begin{array}{l}.00022 \\
.00019\end{array}$ & $\begin{array}{l}.25 \\
.23\end{array}$ & $\begin{array}{l}.0132 \\
.0132\end{array}$ & $\begin{array}{l}13.93 \\
13.93\end{array}$ \\
\hline $\begin{array}{l}40.0 \\
40.0 \\
40.0 \\
40.0 \\
40.0\end{array}$ & $\begin{array}{l}40.0 \\
39.0 \\
38.0 \\
37.0 \\
36.0\end{array}$ & $\begin{array}{l}40.0 \\
38.0 \\
36.0 \\
33.8 \\
31.5\end{array}$ & $\begin{array}{r}100.0 \\
92.6 \\
85.3 \\
78.2 \\
71.1\end{array}$ & $\begin{array}{l}.2477 \\
.2293 \\
.2113 \\
.1936 \\
.1762\end{array}$ & $\begin{array}{l}.00627 \\
.00581 \\
.00535 \\
.00489 \\
.00445\end{array}$ & $\begin{array}{l}16.39 \\
15.89 \\
15.39 \\
14.90 \\
14.43\end{array}$ & $\begin{array}{l}.0469 \\
.0459 \\
.0449 \\
.0439 \\
.0430\end{array}$ & $\begin{array}{l}15.29 \\
15.28 \\
15.27 \\
15.25 \\
15.24\end{array}$ \\
\hline $\begin{array}{l}40.0 \\
40.0 \\
40.0 \\
40.0 \\
40.0\end{array}$ & $\begin{array}{l}35.0 \\
34.0 \\
33.0 \\
32.0 \\
31.0\end{array}$ & $\begin{array}{l}29.3 \\
26.9 \\
24.3 \\
22.9 \\
19.8\end{array}$ & $\begin{array}{l}64.2 \\
57.5 \\
50.8 \\
47.6 \\
41.2\end{array}$ & $\begin{array}{l}.1591 \\
.1423 \\
.1258 \\
.1179 \\
.1020\end{array}$ & $\begin{array}{l}.00402 \\
.00359 \\
.00317 \\
.00297 \\
.00257\end{array}$ & $\begin{array}{l}13.96 \\
13.50 \\
13.05 \\
12.83 \\
12.40\end{array}$ & $\begin{array}{l}.0420 \\
.0411 \\
.0401 \\
.0397 \\
.0388\end{array}$ & $\begin{array}{l}15.23 \\
15.22 \\
15.21 \\
15.21 \\
15.20\end{array}$ \\
\hline $\begin{array}{l}40.0 \\
40.0 \\
40.0 \\
40.0 \\
40.0 \\
40.0\end{array}$ & $\begin{array}{l}30.0 \\
29.0 \\
28.0 \\
27.0 \\
26.0 \\
25.0\end{array}$ & $\begin{array}{r}16.4 \\
12.5 \\
7.8 \\
1.9 \\
-6.1 \\
-19.3\end{array}$ & $\begin{array}{r}34.9 \\
28.7 \\
22.7 \\
16.8 \\
11.0 \\
5.3\end{array}$ & $\begin{array}{l}.0864 \\
.0711 \\
.0562 \\
.0415 \\
.0272 \\
.0131\end{array}$ & $\begin{array}{l}.00217 \\
.00179 \\
.00141 \\
.00104 \\
.00068 \\
.00033\end{array}$ & $\begin{array}{r}11.97 \\
11.56 \\
11.15 \\
10.75 \\
10.36 \\
9.98\end{array}$ & $\begin{array}{l}.0379 \\
.0370 \\
.0361 \\
.0353 \\
.0344 \\
.0336\end{array}$ & $\begin{array}{l}15.19 \\
15.18 \\
15.17 \\
15.16 \\
15.15 \\
15.14\end{array}$ \\
\hline
\end{tabular}


$\mathrm{PB}=24.89$, Altitude $=5000-$ Continued

\begin{tabular}{|c|c|c|c|c|c|c|c|c|}
\hline DB & WB & $\mathrm{DP}$ & $\mathrm{RH}$ & PV & W & $\mathrm{H}$ & $\mathrm{S}$ & $\mathrm{V}$ \\
\hline 80.0 & 80.0 & 80.0 & 100.0 & 1.0323 & .02703 & 48.85 & .1091 & 17.06 \\
\hline 80.0 & 79.0 & 78.7 & 95.9 & .9902 & .02588 & 47.59 & .1068 & 17.03 \\
\hline 80.0 & 78.0 & 77.4 & 91.9 & .9490 & .02476 & 46.37 & .1045 & 17.00 \\
\hline 80.0 & 77.0 & 76.1 & 88.0 & .9087 & .02367 & 45.17 & .1022 & 16.97 \\
\hline 80.0 & 76.0 & 74.8 & 84.2 & .8693 & .02260 & 44.01 & .1001 & 16.94 \\
\hline 80.0 & 75.0 & 73.5 & 80.5 & .8308 & .02157 & 42.87 & .0979 & 16.92 \\
\hline 80.0 & 74.0 & 72.1 & 76.8 & .7932 & .02056 & 41.76 & .0958 & 16.89 \\
\hline 80.0 & 73.0 & 70.7 & 73.3 & .7563 & .01957 & 40.68 & .0938 & 16.86 \\
\hline 80.0 & 72.0 & 69.2 & 69.8 & .7203 & .01861 & 39.63 & .0918 & 16.84 \\
\hline 80.0 & 71.0 & 67.8 & 66.4 & .6850 & .01767 & 38.60 & .0899 & 16.81 \\
\hline 80.0 & 70.0 & 66.3 & 63.0 & .6506 & .01676 & 37.60 & .0880 & 16.79 \\
\hline 80.0 & 69.0 & 64.8 & 59.8 & .6168 & .01587 & 36.63 & .0861 & 16.77 \\
\hline 80.0 & 68.0 & 63.2 & 56.6 & .5838 & .01500 & 35.67 & .0843 & 16.74 \\
\hline 80.0 & 67.0 & 61.6 & 53.4 & .5515 & .01415 & 34.74 & .0825 & 16.72 \\
\hline 80.0 & 66.0 & 59.9 & 50.4 & .5199 & .01332 & 33.84 & .0808 & 16.70 \\
\hline 80.0 & 65.0 & 58.2 & 47.4 & .4890 & .01251 & 32.95 & .0791 & 16.68 \\
\hline 80.0 & 64.0 & 56.4 & 44.4 & .4588 & .01172 & 32.09 & .0774 & 16.66 \\
\hline 80.0 & 63.0 & 54.6 & 41.6 & .4292 & .01095 & 31.24 & .0757 & 16.64 \\
\hline 80.0 & 62.0 & 52.7 & 38.8 & .4002 & .01020 & 30.42 & .0741 & 16.62 \\
\hline 80.0 & 61.0 & 50.7 & 36.0 & .3719 & .00947 & 29.61 & .0726 & 16.60 \\
\hline 80.0 & 60.0 & 48.6 & 33.3 & .3441 & .00875 & 28.83 & .0710 & 16.58 \\
\hline 80.0 & 59.0 & 46.4 & 30.7 & .3169 & .00805 & 28.06 & .0695 & 16.56 \\
\hline 80.0 & 58.0 & 44.1 & 28.1 & .2903 & .00737 & 27.31 & .0680 & 16.54 \\
\hline 80.0 & 57.0 & 41.7 & 25.6 & .2643 & .00670 & 26.58 & .0666 & 16.53 \\
\hline 80.0 & 56.0 & 39.1 & 23.1 & .2388 & .00605 & 25.86 & .0651 & 16.51 \\
\hline 80.0 & 55.0 & 36.3 & 20.7 & .2138 & .00541 & 25.16 & .0637 & 16.49 \\
\hline 80.0 & 54.0 & 33.2 & 18.3 & .1893 & .00478 & 24.48 & .0624 & 16.48 \\
\hline 80.0 & 53.0 & 30.1 & 16.0 & .1653 & .00417 & 23.81 & .0610 & 16.46 \\
\hline 80.0 & 52.0 & 26.8 & 13.7 & .1418 & .00358 & 23.16 & .0597 & 16.44 \\
\hline 80.0 & 51.0 & 23.0 & 11.5 & .1188 & .00299 & 22.52 & .0583 & 16.43 \\
\hline 80.0 & 50.0 & 18.7 & 9.3 & .0963 & .00242 & 21.89 & .0570 & 16.41 \\
\hline 80.0 & 49.0 & 13.3 & 7.2 & .0742 & .00187 & 21.28 & .0557 & 16.40 \\
\hline 80.0 & 48.0 & 6.4 & 5.1 & .0525 & .00132 & 20.68 & .0545 & 16.39 \\
\hline 80.0 & 47.0 & -3.5 & 3.0 & .0313 & .00079 & 20.10 & .0532 & 16.37 \\
\hline 80.0 & 46.0 & -23.3 & 1.0 & .0104 & .00026 & 19.52 & .0519 & 16.36 \\
\hline
\end{tabular}


$\mathrm{PB}=24.89$, Altitude $=5000-$ Continued

\begin{tabular}{|c|c|c|c|c|c|c|c|c|}
\hline $\mathrm{DB}$ & WB & $\mathrm{DP}$ & $\mathrm{RH}$ & PV & W & $\mathrm{H}$ & S & $\mathrm{V}$ \\
\hline 120.0 & 120.0 & 120.0 & 100.0 & 3.4476 & .10058 & 140.80 & .2730 & 20.39 \\
\hline 120.0 & 118.0 & 117.8 & 94.1 & 3.2439 & .09374 & 133.19 & .2598 & 20.20 \\
\hline 120.0 & 116.0 & 115.6 & 88.4 & 3.0489 & .08730 & 126.03 & .2473 & 20.02 \\
\hline 120.0 & 114.0 & 113.4 & 83.0 & 2.8621 & .08125 & 119.30 & .2355 & 19.85 \\
\hline 120.0 & 112.0 & 111.1 & 77.8 & 2.6832 & .07555 & 112.96 & .2244 & 19.69 \\
\hline 120.0 & 110.0 & 108.9 & 72.9 & 2.5120 & .07018 & 106.99 & .2138 & 19.54 \\
\hline 120.0 & 108.0 & 106.5 & 68.1 & 2.3482 & .06512 & 101.36 & .2039 & 19.40 \\
\hline 120.0 & 106.0 & 104.2 & 63.6 & 2.1914 & .06035 & 96.05 & .1944 & 19.26 \\
\hline 120.0 & 104.0 & 101.8 & 59.2 & 2.0415 & .05584 & 91.04 & .1855 & 19.14 \\
\hline 120.0 & 102.0 & 99.4 & 55.1 & 1.8980 & .05159 & 86.30 & .1770 & 19.02 \\
\hline 120.0 & 100.0 & 96.9 & 51.1 & 1.7608 & .04757 & 81.83 & .1689 & 18.90 \\
\hline 120.0 & 98.0 & 94.4 & 47.3 & 1.6296 & .04378 & 77.60 & .1613 & 18.80 \\
\hline 120.0 & 96.0 & 91.8 & 43.6 & 1.5041 & .04019 & 73.61 & .1541 & 18.70 \\
\hline 120.0 & 94.0 & 89.1 & 40.1 & 1.3842 & .03679 & 69.83 & .1472 & 18.60 \\
\hline 120.0 & 92.0 & 86.4 & 36.8 & 1.2695 & .03357 & 66.25 & .1406 & 18.51 \\
\hline 120.0 & 90.0 & 83.6 & 33.6 & 1.1599 & .03053 & 62.86 & .1344 & 18.43 \\
\hline 120.0 & 88.0 & 80.7 & 30.6 & 1.0551 & .02765 & 59.65 & .1284 & 18.34 \\
\hline 120.0 & 86.0 & 77.6 & 27.7 & .9549 & .02492 & 56.61 & 1228 & 18.27 \\
\hline 120.0 & 84.0 & 74.5 & 24.9 & .8591 & .02233 & 53.72 & .1174 & 18.19 \\
\hline 120.0 & 82.0 & 71.1 & 22.3 & .7676 & .01987 & 50.99 & .1123 & 18.13 \\
\hline 120.0 & 80.0 & 67.6 & 19.7 & .6801 & .01754 & 48.39 & .1074 & 18.06 \\
\hline 120.0 & 78.0 & 63.8 & 17.3 & .5965 & .01533 & 45.93 & .1027 & 18.00 \\
\hline 120.0 & 76.0 & 59.7 & 15.0 & .5165 & .01323 & 43.59 & .0982 & 17.94 \\
\hline 120.0 & 74.0 & 55.3 & 12.8 & .4401 & .01124 & 41.37 & .0939 & 17.88 \\
\hline 120.0 & 72.0 & 50.3 & 10.6 & .3669 & .00934 & 39.26 & .0898 & 17.83 \\
\hline 120.0 & 70.0 & 44.7 & 8.6 & .2970 & .00754 & 37.25 & .0858 & 17.78 \\
\hline 120.0 & 68.0 & 38.1 & 6.7 & .2301 & .00583 & 35.34 & .0820 & 17.73 \\
\hline 120.0 & 66.0 & 30.2 & 4.8 & .1661 & .00420 & 33.53 & .0784 & 17.68 \\
\hline 120.0 & 64.0 & 20.4 & 3.0 & .1049 & .00264 & 31.80 & .0748 & 17.64 \\
\hline 120.0 & 62.0 & 4.0 & 1.3 & .0463 & .00116 & 30.15 & .0713 & 17.60 \\
\hline
\end{tabular}


$\mathrm{PB}=24.89$, Altitude $=5000-$ Continued

\begin{tabular}{|c|c|c|c|c|c|c|c|c|}
\hline $\mathrm{DB}$ & WB & $\mathrm{DP}$ & $\mathrm{RH}$ & PV & W & $\mathrm{H}$ & $\mathrm{S}$ & V \\
\hline 160.0 & 160.0 & 160.0 & 100.0 & 9.6555 & .39767 & 487.73 & .8530 & 30.69 \\
\hline 160.0 & 158.0 & 157.9 & 95.2 & 9.1911 & .36727 & 453.44 & .7968 & 29.78 \\
\hline 160.0 & 156.0 & 155.9 & 90.6 & 8.7442 & .33967 & 422.30 & .7456 & 28.96 \\
\hline 160.0 & 154.0 & 153.8 & 86.1 & 8.3141 & .31452 & 393.92 & .6989 & 28.21 \\
\hline 160.0 & 152.0 & 151.7 & 81.8 & 7.9003 & .29154 & 367.97 & .6560 & 27.52 \\
\hline 160.0 & 150.0 & 149.6 & 77.7 & 7.5024 & .27047 & 344.19 & .6166 & 26.89 \\
\hline 160.0 & 148.0 & 147.5 & 73.7 & 7.1198 & 25111 & 322.33 & .5802 & 26.31 \\
\hline 160.0 & 146.0 & 145.4 & 69.9 & 6.7521 & .23327 & 302.19 & .5467 & 25.78 \\
\hline 160.0 & 144.0 & 143.3 & 66.3 & 6.3987 & .21680 & 283.59 & .5155 & 25.29 \\
\hline 160.0 & 142.0 & 141.1 & 62.8 & 6.0593 & 20156 & 266.38 & .4867 & 24.83 \\
\hline 160.0 & 140.0 & 139.0 & 59.4 & 5.7332 & .18744 & 250.43 & .4598 & 24.41 \\
\hline 160.0 & 138.0 & 136.8 & 56.1 & 5.4202 & .17433 & 235.62 & .4348 & 24.02 \\
\hline 160.0 & 136.0 & 134.6 & 53.0 & 5.1197 & .16214 & 221.84 & .4115 & 23.65 \\
\hline 160.0 & 134.0 & 132.4 & 50.0 & 4.8313 & .15078 & 209.01 & .3896 & 23.31 \\
\hline 160.0 & 132.0 & 130.2 & 47.2 & 4.5547 & .14019 & 197.05 & .3692 & 2299 \\
\hline 160.0 & 130.0 & 128.0 & 44.4 & 4.2893 & .13031 & 185.87 & .3501 & 22.70 \\
\hline 160.0 & 128.0 & 125.7 & 41.8 & 4.0348 & .12106 & 175.43 & .3322 & 22.42 \\
\hline 160.0 & 126.0 & 123.4 & 39.3 & 3.7909 & .11241 & 165.65 & .3153 & 22.16 \\
\hline 160.0 & 124.0 & 121.1 & 36.8 & 3.5570 & .10431 & 156.49 & .2995 & 21.92 \\
\hline 160.0 & 122.0 & 118.8 & 34.5 & 3.3330 & .09671 & 147.89 & .2846 & 21.69 \\
\hline 160.0 & 120.0 & 116.4 & 32.3 & 3.1183 & .08958 & 139.83 & .2705 & 21.47 \\
\hline 160.0 & 118.0 & 114.0 & 30.2 & 2.9127 & .08288 & 132.25 & .2573 & 21.27 \\
\hline 160.0 & 116.0 & 111.6 & 28.1 & 2.7158 & .07658 & 125.13 & .2448 & 21.08 \\
\hline 160.0 & 114.0 & 109.1 & 26.2 & 2.5273 & .07066 & 118.43 & .2330 & 20.91 \\
\hline 160.0 & 112.0 & 106.5 & 24.3 & 2.3468 & .06508 & 112.12 & .2218 & 20.74 \\
\hline 160.0 & 110.0 & 103.9 & 22.5 & 2.1741 & .05983 & 106.18 & .2113 & 20.58 \\
\hline 160.0 & 108.0 & 101.3 & 20.8 & 2.0089 & .05487 & 100.58 & .2013 & 20.43 \\
\hline 160.0 & 106.0 & 98.6 & 19.2 & 1.8508 & .05020 & 95.30 & .1918 & 20.29 \\
\hline 160.0 & 104.0 & 95.8 & 17.6 & 1.6996 & .04580 & 90.31 & .1828 & 20.16 \\
\hline 160.0 & 102.0 & 92.9 & 16.1 & 1.5550 & .04164 & 85.61 & .1743 & 20.03 \\
\hline 160.0 & 100.0 & 89.9 & 14.7 & 1.4168 & .03771 & 81.16 & .1662 & 19.91 \\
\hline 160.0 & 98.0 & 86.8 & 13.3 & 1.2846 & .03400 & 76.96 & .1586 & 19.80 \\
\hline 160.0 & 96.0 & 83.5 & 12.0 & 1.1582 & .03049 & 72.99 & .1513 & 19.70 \\
\hline 160.0 & 94.0 & 80.2 & 10.7 & 1.0374 & .02717 & 69.23 & .1443 & 19.60 \\
\hline 160.0 & 92.0 & 76.6 & 9.5 & .9219 & .02402 & 65.67 & .1377 & 19.50 \\
\hline 160.0 & 90.0 & 72.8 & 8.4 & .8116 & .02105 & 62.31 & .1314 & 19.41 \\
\hline 160.0 & 88.0 & 68.7 & 7.3 & .7062 & .01823 & 59.12 & .1254 & 19.33 \\
\hline 160.0 & 86.0 & 64.2 & 6.3 & .6054 & .01557 & 56.10 & .1197 & 19.25 \\
\hline 160.0 & 84.0 & 59.3 & 5.3 & .5091 & .01304 & 53.24 & .1142 & 19.17 \\
\hline 160.0 & 82.0 & 53.8 & 4.3 & .4171 & .01064 & 50.53 & .1090 & 19.10 \\
\hline 160.0 & 80.0 & 47.4 & 3.4 & .3292 & .00837 & 47.95 & .1040 & 19.03 \\
\hline 160.0 & 78.0 & 39.7 & 2.5 & .2452 & .00621 & 45.51 & .0991 & 18.97 \\
\hline 160.0 & 76.0 & 30.0 & 1.7 & .1649 & .00416 & 43.19 & .0945 & 18.90 \\
\hline 160.0 & 74.0 & 16.8 & .9 & .0881 & .00222 & 40.99 & .0899 & 18.85 \\
\hline 160.0 & 72.0 & -17.2 & .2 & .0148 & .00037 & 38.90 & .0854 & 18.79 \\
\hline
\end{tabular}


$\mathrm{PB}=22.65$, Altitude $=7500$

\begin{tabular}{|c|c|c|c|c|c|c|c|c|}
\hline DB & WB & $\mathrm{DP}$ & $\mathrm{RH}$ & PV & W & $\mathrm{H}$ & $\mathrm{S}$ & $\mathrm{V}$ \\
\hline-40.0 & -40.0 & -40.0 & 100.0 & .0038 & .00010 & -9.46 & -.0024 & 13.96 \\
\hline $\begin{array}{l}.0 \\
.0 \\
.0 \\
.0 \\
.0\end{array}$ & $\begin{array}{r}.0 \\
-.1 \\
-.2 \\
-.3 \\
-.4\end{array}$ & $\begin{array}{r}.0 \\
-.5 \\
-.9 \\
-1.4 \\
-1.9\end{array}$ & $\begin{array}{r}100.0 \\
97.6 \\
95.2 \\
92.8 \\
90.3\end{array}$ & $\begin{array}{l}.0376 \\
.0367 \\
.0358 \\
.0349 \\
.0340\end{array}$ & $\begin{array}{l}.00104 \\
.00101 \\
.00099 \\
.00096 \\
.00094\end{array}$ & $\begin{array}{l}1.13 \\
1.11 \\
1.08 \\
1.05 \\
1.03\end{array}$ & $\begin{array}{l}.0217 \\
.0216 \\
.0216 \\
.0215 \\
.0214\end{array}$ & $\begin{array}{l}15.32 \\
15.32 \\
15.32 \\
15.32 \\
15.32\end{array}$ \\
\hline $\begin{array}{l}.0 \\
.0 \\
.0 \\
.0 \\
.0\end{array}$ & $\begin{array}{l}-.5 \\
-.6 \\
-.7 \\
-.8 \\
-.9\end{array}$ & $\begin{array}{l}-2.4 \\
-3.0 \\
-3.5 \\
-4.1 \\
-4.6\end{array}$ & $\begin{array}{l}87.9 \\
85.5 \\
83.1 \\
80.7 \\
78.3\end{array}$ & $\begin{array}{l}.0331 \\
.0322 \\
.0313 \\
.0304 \\
.0295\end{array}$ & $\begin{array}{l}.00091 \\
.00089 \\
.00086 \\
.00084 \\
.00081\end{array}$ & $\begin{array}{r}1.00 \\
.97 \\
.95 \\
.92 \\
.89\end{array}$ & $\begin{array}{l}.0214 \\
.0213 \\
.0213 \\
.0212 \\
.0212\end{array}$ & $\begin{array}{l}15.32 \\
15.32 \\
15.32 \\
15.32 \\
15.32\end{array}$ \\
\hline $\begin{array}{l}.0 \\
.0 \\
.0 \\
.0 \\
.0\end{array}$ & $\begin{array}{l}-1.0 \\
-1.1 \\
-1.2 \\
-1.3 \\
-1.4\end{array}$ & $\begin{array}{l}-5.2 \\
-5.8 \\
-6.4 \\
-7.0 \\
-7.7\end{array}$ & $\begin{array}{l}75.9 \\
73.5 \\
71.1 \\
68.8 \\
66.4\end{array}$ & $\begin{array}{l}.0286 \\
.0277 \\
.0268 \\
.0259 \\
.0250\end{array}$ & $\begin{array}{l}.00079 \\
.00076 \\
.00074 \\
.00071 \\
.00069\end{array}$ & $\begin{array}{l}.87 \\
.84 \\
.82 \\
.79 \\
.76\end{array}$ & $\begin{array}{l}.0211 \\
.0210 \\
.0210 \\
.0209 \\
.0209\end{array}$ & $\begin{array}{l}15.32 \\
15.32 \\
15.32 \\
15.32 \\
15.32\end{array}$ \\
\hline $\begin{array}{l}.0 \\
.0 \\
.0 \\
.0 \\
.0\end{array}$ & $\begin{array}{l}-1.5 \\
-1.6 \\
-1.7 \\
-1.8 \\
-1.9\end{array}$ & $\begin{array}{r}-8.4 \\
-9.1 \\
-9.8 \\
-10.5 \\
-11.3\end{array}$ & $\begin{array}{l}64.0 \\
61.6 \\
59.2 \\
56.9 \\
54.5\end{array}$ & $\begin{array}{l}.0241 \\
.0232 \\
.0223 \\
.0214 \\
.0205\end{array}$ & $\begin{array}{l}.00066 \\
.00064 \\
.00062 \\
.00059 \\
.00057\end{array}$ & $\begin{array}{l}.74 \\
.71 \\
.68 \\
.66 \\
.63\end{array}$ & $\begin{array}{l}.0208 \\
.0207 \\
.0207 \\
.0206 \\
.0206\end{array}$ & $\begin{array}{l}15.31 \\
15.31 \\
15.31 \\
15.31 \\
15.31\end{array}$ \\
\hline $\begin{array}{l}.0 \\
.0 \\
.0 \\
.0 \\
.0\end{array}$ & $\begin{array}{l}-2.0 \\
-2.1 \\
-2.2 \\
-2.3 \\
-2.4\end{array}$ & $\begin{array}{l}-12.1 \\
-13.0 \\
-13.8 \\
-14.7 \\
-15.7\end{array}$ & $\begin{array}{l}52.1 \\
49.7 \\
47.4 \\
45.0 \\
42.6\end{array}$ & $\begin{array}{l}.0196 \\
.0187 \\
.0178 \\
.0169 \\
.0161\end{array}$ & $\begin{array}{l}.00054 \\
.00052 \\
.00049 \\
.00047 \\
.00044\end{array}$ & $\begin{array}{l}.61 \\
.58 \\
.55 \\
.53 \\
.50\end{array}$ & $\begin{array}{l}.0205 \\
.0204 \\
.0204 \\
.0203 \\
.0203\end{array}$ & $\begin{array}{l}15.31 \\
15.31 \\
15.31 \\
15.31 \\
15.31\end{array}$ \\
\hline $\begin{array}{l}.0 \\
.0 \\
.0 \\
.0 \\
.0\end{array}$ & $\begin{array}{l}-2.5 \\
-2.6 \\
-2.7 \\
-2.8 \\
-2.9\end{array}$ & $\begin{array}{l}-16.7 \\
-17.8 \\
-18.9 \\
-20.1 \\
-21.4\end{array}$ & $\begin{array}{l}40.3 \\
37.9 \\
35.6 \\
33.2 \\
30.9\end{array}$ & $\begin{array}{l}.0152 \\
.0143 \\
.0134 \\
.0125 \\
.0116\end{array}$ & $\begin{array}{l}.00042 \\
.00039 \\
.00037 \\
.00034 \\
.00032\end{array}$ & $\begin{array}{l}.48 \\
.45 \\
.42 \\
.40 \\
.37\end{array}$ & $\begin{array}{l}.0202 \\
.0201 \\
.0201 \\
.0200 \\
.0200\end{array}$ & $\begin{array}{l}15.31 \\
15.31 \\
15.31 \\
15.31 \\
15.31\end{array}$ \\
\hline $\begin{array}{l}.0 \\
.0 \\
.0 \\
.0 \\
.0\end{array}$ & $\begin{array}{l}-3.0 \\
-3.1 \\
-3.2 \\
-3.3 \\
-3.4\end{array}$ & $\begin{array}{l}-22.8 \\
-24.2 \\
-25.8 \\
-27.6 \\
-29.5\end{array}$ & $\begin{array}{l}28.5 \\
26.2 \\
23.8 \\
21.5 \\
19.1\end{array}$ & $\begin{array}{l}.0107 \\
.0099 \\
.0090 \\
.0081 \\
.0072\end{array}$ & $\begin{array}{l}.00030 \\
.00027 \\
.00025 \\
.00022 \\
.00020\end{array}$ & $\begin{array}{l}.35 \\
.32 \\
.29 \\
.27 \\
.24\end{array}$ & $\begin{array}{l}.0199 \\
.0198 \\
.0198 \\
.0197 \\
.0197\end{array}$ & $\begin{array}{l}15.31 \\
15.31 \\
15.30 \\
15.30 \\
15.30\end{array}$ \\
\hline
\end{tabular}


$\mathrm{PB}=22.65$, Altitude $=7500-$ Continued

$\begin{array}{lrrrrrrrr}\text { DB } & \text { WB } & \text { DP } & \text { RH } & \text { PV } & \text { W } & \text { H } & \text { S } & \text { V } \\ & & & & & & & & \\ 40.0 & 40.0 & 40.0 & 100.0 & .2477 & .00690 & 17.08 & .0548 & 16.82 \\ 40.0 & 39.0 & 38.1 & 92.9 & .2301 & .00641 & 16.54 & .0538 & 16.80 \\ 40.0 & 38.0 & 36.1 & 86.0 & .2129 & .00592 & 16.02 & .0527 & 16.79 \\ 40.0 & 37.0 & 34.1 & 79.1 & .1960 & .00545 & 15.51 & .0517 & 16.78 \\ 40.0 & 36.0 & 31.9 & 72.4 & .1794 & .00498 & 15.01 & .0507 & 16.77 \\ 40.0 & 35.0 & 29.8 & 65.9 & .1631 & .00453 & 14.52 & .0497 & 16.75 \\ 40.0 & 34.0 & 27.6 & 59.4 & .1471 & .00408 & 14.04 & .0487 & 16.74 \\ 40.0 & 33.0 & 25.2 & 53.1 & .1314 & .00364 & 13.56 & .0477 & 16.73 \\ 40.0 & 32.0 & 23.9 & 49.9 & .1235 & .00342 & 13.33 & .0472 & 16.73 \\ 40.0 & 31.0 & 21.1 & 43.7 & .1083 & .00300 & 12.87 & .0463 & 16.71 \\ 40.0 & 30.0 & 18.0 & 37.7 & .0935 & .00259 & 12.42 & .0453 & 16.70 \\ 40.0 & 29.0 & 14.6 & 31.9 & .0789 & .00218 & 11.99 & .0444 & 16.69 \\ 40.0 & 28.0 & 10.6 & 26.1 & .0647 & .00179 & 11.56 & .0435 & 16.68 \\ 40.0 & 27.0 & 5.8 & 20.5 & .0507 & .00140 & 11.15 & .0426 & 16.67 \\ 40.0 & 26.0 & -.3 & 15.0 & .0370 & .00102 & 10.74 & .0417 & 16.66 \\ 40.0 & 25.0 & -8.7 & 9.6 & .0237 & .00065 & 10.34 & .0408 & 16.65 \\ 40.0 & 24.0 & -23.1 & 4.2 & .0105 & .00029 & 9.95 & .0399 & 16.64\end{array}$


PB $=22.65$, Altitude $=7500-$ Continued

\begin{tabular}{|c|c|c|c|c|c|c|c|c|}
\hline DB & WB & $\mathrm{DP}$ & $\mathrm{RH}$ & PV & W & $\mathrm{H}$ & $\mathrm{S}$ & V \\
\hline 80.0 & 80.0 & 80.0 & 100.0 & 1.0323 & .02982 & 51.92 & .1216 & 18.83 \\
\hline 80.0 & 79.0 & 78.8 & 96.0 & .9910 & .02857 & 50.55 & .1190 & 18.79 \\
\hline 80.0 & 78.0 & 77.5 & 92.1 & .9506 & .02735 & 49.22 & .1165 & 18.76 \\
\hline 80.0 & 77.0 & 76.2 & 88.3 & .9112 & .02617 & 47.92 & .1141 & 18.72 \\
\hline 80.0 & 76.0 & 74.9 & 84.5 & .8726 & .02502 & 46.66 & .1117 & 18.69 \\
\hline 80.0 & 75.0 & 73.6 & 80.9 & .8349 & .02390 & 45.43 & .1094 & 18.66 \\
\hline 80.0 & 74.0 & 72.3 & 77.3 & .7981 & .02280 & 44.23 & .1072 & 18.63 \\
\hline 80.0 & 73.0 & 70.9 & 73.8 & .7620 & .02174 & 43.07 & .1050 & 18.59 \\
\hline 80.0 & 72.0 & 69.5 & 70.4 & .7268 & .02070 & 41.93 & .1028 & 18.56 \\
\hline 80.0 & 71.0 & 68.1 & 67.1 & .6924 & .01969 & 40.82 & .1007 & 18.54 \\
\hline 80.0 & 70.0 & 66.6 & 63.8 & .6587 & .01870 & 39.74 & .0987 & 18.51 \\
\hline 80.0 & 69.0 & 65.2 & 60.6 & .6258 & .01774 & 38.68 & .0967 & 18.48 \\
\hline 80.0 & 68.0 & 63.7 & 57.5 & .5936 & .01680 & 37.66 & .0947 & 18.45 \\
\hline 80.0 & 67.0 & 62.1 & 54.5 & .5621 & .01589 & 36.66 & .0928 & 18.43 \\
\hline 80.0 & 66.0 & 60.5 & 51.5 & .5314 & .01500 & 35.68 & .0909 & 18.40 \\
\hline 80.0 & 65.0 & 58.9 & 48.6 & .5013 & .01413 & 34.73 & .0891 & 18.38 \\
\hline 80.0 & 64.0 & 57.2 & 45.7 & .4718 & .01328 & 33.80 & .0873 & 18.35 \\
\hline 80.0 & 63.0 & 55.5 & 42.9 & .4430 & .01245 & 32.89 & .0855 & 18.33 \\
\hline 80.0 & 62.0 & 53.7 & 40.2 & .4149 & .01165 & 32.01 & .0838 & 18.30 \\
\hline 80.0 & 61.0 & 51.8 & 37.5 & .3873 & .01086 & 31.15 & .0821 & 18.28 \\
\hline 80.0 & 60.0 & 49.8 & 34.9 & .3603 & .01009 & 30.30 & .0805 & 18.26 \\
\hline 80.0 & 59.0 & 47.8 & 32.4 & .3340 & .00934 & 29.48 & .0789 & 18.24 \\
\hline 80.0 & 58.0 & 45.7 & 29.9 & .3082 & .00861 & 28.68 & .0773 & 18.22 \\
\hline 80.0 & 57.0 & 43.4 & 27.4 & .2829 & .00789 & 27.90 & .0757 & 18.20 \\
\hline 80.0 & 56.0 & 41.1 & 25.0 & .2582 & .00720 & 27.13 & .0742 & 18.18 \\
\hline 80.0 & 55.0 & 38.5 & 22.7 & .2340 & .00652 & 26.39 & .0727 & 18.16 \\
\hline 80.0 & 54.0 & 35.8 & 20.4 & .2103 & .00585 & 25.66 & .0713 & 18.14 \\
\hline 80.0 & 53.0 & 32.9 & 18.1 & .1872 & .00520 & 24.94 & .0698 & 18.12 \\
\hline 80.0 & 52.0 & 30.0 & 15.9 & .1645 & .00456 & 24.25 & .0684 & 18.10 \\
\hline 80.0 & 51.0 & 26.9 & 13.8 & .1422 & .00394 & 23.57 & .0670 & 18.08 \\
\hline 80.0 & 50.0 & 23.3 & 11.7 & .1205 & .00334 & 22.90 & .0656 & 18.06 \\
\hline 80.0 & 49.0 & 19.3 & 9.6 & .0992 & .00274 & 22.25 & .0643 & 18.05 \\
\hline 80.0 & 48.0 & 14.4 & 7.6 & .0783 & .00217 & 21.62 & .0629 & 18.03 \\
\hline 80.0 & 47.0 & 8.4 & 5.6 & .0578 & .00160 & 20.99 & .0616 & 18.01 \\
\hline 80.0 & 46.0 & .1 & 3.7 & .0378 & .00104 & 20.39 & .0603 & 18.00 \\
\hline 80.0 & 45.0 & -13.5 & 1.8 & .0182 & .00050 & 19.79 & .0590 & 17.98 \\
\hline
\end{tabular}


$\mathrm{PB}=22.65$, Altitude $=7500-$ Continued

\begin{tabular}{|c|c|c|c|c|c|c|c|c|}
\hline $\mathrm{DB}$ & WB & $\mathrm{DP}$ & $\mathrm{RH}$ & PV & W & $\mathrm{H}$ & $\mathrm{S}$ & $\mathrm{V}$ \\
\hline 120.0 & 120.0 & 120.0 & 100.0 & 3.4476 & .11229 & 153.84 & .3032 & 22.77 \\
\hline 120.0 & 118.0 & 117.8 & 94.1 & 3.2456 & .10460 & 145.29 & .2884 & 22.53 \\
\hline 120.0 & 116.0 & 115.7 & 88.5 & 3.0522 & .09738 & 137.26 & .2744 & 22.31 \\
\hline 120.0 & 114.0 & 113.4 & 83.2 & 2.8671 & .09061 & 129.73 & .2612 & 22.10 \\
\hline 120.0 & 112.0 & 111.2 & 78.0 & 2.6899 & .08425 & 122.65 & .2487 & 21.91 \\
\hline 120.0 & 110.0 & 109.0 & 73.1 & 2.5204 & .07826 & 116.00 & .2370 & 21.72 \\
\hline 120.0 & 108.0 & 106.7 & 68.4 & 2.3582 & .07264 & 109.73 & .2259 & 21.55 \\
\hline 120.0 & 106.0 & 104.4 & 63.9 & 2.2031 & .06734 & 103.84 & .2154 & 21.38 \\
\hline 120.0 & 104.0 & 102.0 & 59.6 & 2.0548 & .06234 & 98.28 & .2055 & 21.23 \\
\hline 120.0 & 102.0 & 99.6 & 55.5 & 1.9130 & .05764 & 93.05 & .1961 & 21.09 \\
\hline 120.0 & 100.0 & 97.2 & 51.6 & 1.7774 & .05320 & 88.11 & .1872 & 20.95 \\
\hline 120.0 & 98.0 & 94.7 & 47.8 & 1.6478 & .04902 & 83.45 & .1788 & 20.82 \\
\hline 120.0 & 96.0 & 92.2 & 44.2 & 1.5240 & .04506 & 79.05 & .1708 & 20.70 \\
\hline 120.0 & 94.0 & 89.6 & 40.8 & 1.4057 & .04133 & 74.89 & .1633 & 20.58 \\
\hline 120.0 & 92.0 & 87.0 & 37.5 & 1.2926 & .03780 & 70.96 & .1561 & 20.47 \\
\hline 120.0 & 90.0 & 84.2 & 34.4 & 1.1846 & .03447 & 67.25 & 1493 & 20.37 \\
\hline 120.0 & 88.0 & 81.4 & 31.4 & 1.0814 & .03131 & 63.73 & .1428 & 20.27 \\
\hline 120.0 & 86.0 & 78.5 & 28.5 & .9829 & .02833 & 60.41 & .1366 & 20.18 \\
\hline 120.0 & 84.0 & 75.5 & 25.8 & .8887 & .02550 & 57.26 & .1307 & 20.09 \\
\hline 120.0 & 82.0 & 72.3 & 23.2 & .7988 & .02282 & 54.28 & .1252 & 20.01 \\
\hline 120.0 & 80.0 & 68.9 & 20.7 & .7129 & .02029 & 51.46 & .1198 & 19.93 \\
\hline 120.0 & 78.0 & 65.4 & 18.3 & .6309 & .01789 & 48.78 & .1148 & 19.86 \\
\hline 120.0 & 76.0 & 61.6 & 16.0 & .5525 & .01561 & 46.25 & .1099 & 19.79 \\
\hline 120.0 & 74.0 & 57.5 & 13.9 & .4777 & .01345 & 43.84 & .1053 & 19.72 \\
\hline 120.0 & 72.0 & 53.1 & 11.8 & .4061 & .01140 & 41.55 & .1008 & 19.66 \\
\hline 120.0 & 70.0 & 48.1 & 9.8 & .3378 & .00945 & 39.39 & .0966 & 19.60 \\
\hline 120.0 & 68.0 & 42.5 & 7.9 & .2725 & .00760 & 37.33 & .0925 & 19.54 \\
\hline 120.0 & 66.0 & 35.8 & 6.1 & .2101 & .00584 & 35.37 & .0886 & 19.48 \\
\hline 120.0 & 64.0 & 28.1 & 4.4 & .1504 & .00417 & 33.51 & .0848 & 19.43 \\
\hline 120.0 & 62.0 & 18.0 & 2.7 & .0933 & .00258 & 31.74 & .0812 & 19.38 \\
\hline 120.0 & 60.0 & .6 & 1.1 & .0388 & .00107 & 30.05 & .0776 & 19.34 \\
\hline
\end{tabular}


$\mathrm{PB}=22.65$, Altitude $=7500-$ Continued

\begin{tabular}{|c|c|c|c|c|c|c|c|c|}
\hline $\mathrm{DB}$ & WB & $\mathrm{DP}$ & $\mathrm{RH}$ & PV & W & $\mathrm{H}$ & S & $\mathrm{V}$ \\
\hline 160.0 & 160.0 & 160.0 & 100.0 & 9.6555 & 46613 & 565.09 & .9904 & 35.98 \\
\hline 160.0 & 158.0 & 157.9 & 95.2 & 9.1929 & .42845 & 522.58 & .9208 & 34.74 \\
\hline 160.0 & 156.0 & 155.9 & 90.6 & 8.7477 & .39457 & 484.34 & .8580 & 33.63 \\
\hline 160.0 & 154.0 & 153.8 & 86.2 & 8.3193 & .36396 & 449.80 & .8011 & 32.63 \\
\hline 160.0 & 152.0 & 151.8 & 81.9 & 7.9073 & .33620 & 418.47 & .7493 & 31.71 \\
\hline 160.0 & 150.0 & 149.7 & 77.8 & 7.5111 & .31094 & 389.95 & .7020 & 30.89 \\
\hline 160.0 & 148.0 & 147.6 & 73.8 & 7.1302 & .28788 & 363.91 & .6587 & 30.13 \\
\hline 160.0 & 146.0 & 145.5 & 70.1 & 6.7642 & .26676 & 340.07 & .6190 & 29.43 \\
\hline 160.0 & 144.0 & 143.4 & 66.4 & 6.4125 & .24738 & 318.17 & .5823 & 28.80 \\
\hline 160.0 & 142.0 & 141.2 & 62.9 & 6.0748 & .22953 & 298.01 & .5485 & 28.21 \\
\hline 160.0 & 140.0 & 139.1 & 59.6 & 5.7504 & .21307 & 279.42 & .5172 & 27.67 \\
\hline 160.0 & 138.0 & 137.0 & 56.3 & 5.4390 & .19786 & 262.23 & .4882 & 27.17 \\
\hline 160.0 & 136.0 & 134.8 & 53.2 & 5.1402 & .18377 & 246.31 & .4612 & 26.70 \\
\hline 160.0 & 134.0 & 132.6 & 50.3 & 4.8535 & .17070 & 231.54 & .4361 & 26.27 \\
\hline 160.0 & 132.0 & 130.4 & 47.4 & 4.5785 & .15855 & 217.82 & .4127 & 25.87 \\
\hline 160.0 & 130.0 & 128.2 & 44.7 & 4.3148 & .14725 & 205.04 & .3909 & 25.50 \\
\hline 160.0 & 128.0 & 126.0 & 42.1 & 4.0619 & .13671 & 193.14 & .3704 & 25.15 \\
\hline 160.0 & 126.0 & 123.7 & 39.6 & 3.8196 & .12689 & 182.03 & .3513 & 24.83 \\
\hline 160.0 & 124.0 & 121.4 & 37.2 & 3.5874 & 11771. & 171.65 & .3334 & 24.53 \\
\hline 160.0 & 122.0 & 119.1 & 34.9 & 3.3650 & 10912 & 161.94 & .3165 & 24.24 \\
\hline 160.0 & 120.0 & 116.8 & 32.6 & 3.1519 & .10108 & 152.85 & .3007 & 23.98 \\
\hline 160.0 & 118.0 & 114.4 & 30.5 & 2.9479 & .09355 & 144.34 & .2858 & 23.73 \\
\hline 160.0 & 116.0 & 112.0 & 28.5 & 2.7526 & .08649 & 136.35 & .2718 & 23.50 \\
\hline 160.0 & 114.0 & 109.6 & 26.6 & 2.5658 & .07985 & 128.85 & .2586 & 23.28 \\
\hline 160.0 & 112.0 & 107.1 & 24.7 & 2.3869 & .07362 & 121.80 & .2461 & 23.07 \\
\hline 160.0 & 110.0 & 104.6 & 22.9 & 2.2159 & .06777 & 115.18 & .2344 & 22.88 \\
\hline 160.0 & 108.0 & 102.0 & 21.3 & 2.0522 & .06226 & 108.94 & .2233 & 22.70 \\
\hline 160.0 & 106.0 & 99.3 & 19.6 & 1.8958 & .05707 & 103.08 & .2128 & 22.53 \\
\hline 160.0 & 104.0 & 96.6 & 18.1 & 1.7462 & .05219 & 97.55 & .2028 & 22.36 \\
\hline 160.0 & 102.0 & 93.9 & 16.6 & 1.6032 & .04758 & 92.34 & .1934 & 22.21 \\
\hline 160.0 & 100.0 & 91.0 & 15.2 & 1.4665 & .04324 & 87.43 & .1845 & 22.07 \\
\hline 160.0 & 98.0 & 88.0 & 13.8 & 1.3359 & .03915 & 82.79 & .1761 & 21.93 \\
\hline 160.0 & 96.0 & 84.9 & 12.5 & 1.2111 & .03528 & 78.42 & .1681 & 21.80 \\
\hline 160.0 & 94.0 & 81.7 & 11.3 & 1.0919 & .03163 & 74.29 & .1605 & 21.68 \\
\hline 160.0 & 92.0 & 78.4 & 10.1 & .9780 & .02818 & 70.38 & .1532 & 21.57 \\
\hline 160.0 & 90.0 & 74.8 & 9.0 & .8693 & .02492 & 66.69 & .1463 & 21.46 \\
\hline 160.0 & 88.0 & 71.0 & 7.9 & .7654 & .02184 & 63.20 & .1398 & 21.36 \\
\hline 160.0 & 86.0 & 67.0 & 6.9 & .6662 & .01892 & 59.90 & .1336 & 21.26 \\
\hline 160.0 & 84.0 & 62.6 & 5.9 & .5715 & .01616 & 56.78 & .1276 & 21.17 \\
\hline 160.0 & 82.0 & 57.7 & 5.0 & .4811 & .01355 & 53.82 & .1219 & 21.09 \\
\hline 160.0 & 80.0 & 52.3 & 4.1 & .3947 & .01107 & 51.02 & .1165 & 21.00 \\
\hline 160.0 & 78.0 & 46.0 & 3.2 & .3123 & .00872 & 48.36 & .1113 & 20.93 \\
\hline 160.0 & 76.0 & 38.5 & 2.4 & .2335 & .00650 & 45.85 & .1063 & 20.85 \\
\hline 160.0 & 74.0 & 29.2 & 1.6 & .1583 & .00439 & 43.46 & .1015 & 20.78 \\
\hline 160.0 & 72.0 & 16.5 & .9 & .0865 & .00239 & 41.19 & .0969 & 20.72 \\
\hline 160.0 & 70.0 & -13.7 & .2 & .0179 & .00049 & 39.04 & .0922 & 20.65 \\
\hline
\end{tabular}


$\mathrm{PB}=20.58$, Altitude $=10000$

\begin{tabular}{|c|c|c|c|c|c|c|c|c|}
\hline $\mathrm{DB}$ & WB & DP & $\mathrm{RH}$ & PV & W & $\mathrm{H}$ & $\mathrm{S}$ & $\mathrm{V}$ \\
\hline-40.0 & -40.0 & -40.0 & 100.0 & .0038 & .00011 & -9.44 & .0042 & 15.37 \\
\hline $\begin{array}{l}.0 \\
.0 \\
.0 \\
.0 \\
.0\end{array}$ & $\begin{array}{r}.0 \\
-.1 \\
-.2 \\
-.3 \\
-.4\end{array}$ & $\begin{array}{r}.0 \\
-.4 \\
-.9 \\
-1.3 \\
-1.8\end{array}$ & $\begin{array}{r}100.0 \\
97.8 \\
95.5 \\
93.3 \\
91.0\end{array}$ & $\begin{array}{l}.0376 \\
.0368 \\
.0360 \\
.0351 \\
.0343\end{array}$ & $\begin{array}{l}.00114 \\
.00112 \\
.00109 \\
.00107 \\
.00104\end{array}$ & $\begin{array}{l}1.25 \\
1.23 \\
1.20 \\
1.17 \\
1.14\end{array}$ & $\begin{array}{l}.0285 \\
.0285 \\
.0284 \\
.0283 \\
.0283\end{array}$ & $\begin{array}{l}16.87 \\
16.87 \\
16.87 \\
16.87 \\
16.87\end{array}$ \\
\hline $\begin{array}{l}.0 \\
.0 \\
.0 \\
.0 \\
.0\end{array}$ & $\begin{array}{r}-.5 \\
-.6 \\
-.7 \\
-.8 \\
-.9\end{array}$ & $\begin{array}{l}-2.3 \\
-2.7 \\
-3.2 \\
-3.7 \\
-4.2\end{array}$ & $\begin{array}{l}88.8 \\
86.6 \\
84.3 \\
82.1 \\
79.9\end{array}$ & $\begin{array}{l}.0334 \\
.0326 \\
.0317 \\
.0309 \\
.0301\end{array}$ & $\begin{array}{l}.00102 \\
.00099 \\
.00096 \\
.00094 \\
.00091\end{array}$ & $\begin{array}{l}1.12 \\
1.09 \\
1.06 \\
1.04 \\
1.01\end{array}$ & $\begin{array}{l}.0282 \\
.0282 \\
.0281 \\
.0280 \\
.0280\end{array}$ & $\begin{array}{l}16.87 \\
16.87 \\
16.86 \\
16.86 \\
16.86\end{array}$ \\
\hline $\begin{array}{l}.0 \\
.0 \\
.0 \\
.0 \\
.0\end{array}$ & $\begin{array}{l}-1.0 \\
-1.1 \\
-1.2 \\
-1.3 \\
-1.4\end{array}$ & $\begin{array}{l}-4.8 \\
-5.3 \\
-5.9 \\
-6.4 \\
-7.0\end{array}$ & $\begin{array}{l}77.7 \\
75.4 \\
73.2 \\
71.0 \\
68.8\end{array}$ & $\begin{array}{l}.0292 \\
.0284 \\
.0276 \\
.0267 \\
.0259\end{array}$ & $\begin{array}{l}.00089 \\
.00086 \\
.00084 \\
.00081 \\
.00079\end{array}$ & $\begin{array}{l}.98 \\
.96 \\
.93 \\
.90 \\
.88\end{array}$ & $\begin{array}{l}.0279 \\
.0279 \\
.0278 \\
.0277 \\
.0277\end{array}$ & $\begin{array}{l}16.86 \\
16.86 \\
16.86 \\
16.86 \\
16.86\end{array}$ \\
\hline $\begin{array}{l}.0 \\
.0 \\
.0 \\
.0 \\
.0\end{array}$ & $\begin{array}{l}-1.5 \\
-1.6 \\
-1.7 \\
-1.8 \\
-1.9\end{array}$ & $\begin{array}{r}-7.6 \\
-8.3 \\
-8.9 \\
-9.6 \\
-10.2\end{array}$ & $\begin{array}{l}66.6 \\
64.4 \\
62.2 \\
60.0 \\
57.8\end{array}$ & $\begin{array}{l}.0251 \\
.0242 \\
.0234 \\
.0226 \\
.0217\end{array}$ & $\begin{array}{l}.00076 \\
.00074 \\
.00071 \\
.00069 \\
.00066\end{array}$ & $\begin{array}{l}.85 \\
.82 \\
.79 \\
.77 \\
.74\end{array}$ & $\begin{array}{l}.0276 \\
.0276 \\
.0275 \\
.0274 \\
.0274\end{array}$ & $\begin{array}{l}16.86 \\
16.86 \\
16.86 \\
16.86 \\
16.86\end{array}$ \\
\hline $\begin{array}{l}.0 \\
.0 \\
.0 \\
.0 \\
.0\end{array}$ & $\begin{array}{l}-2.0 \\
-2.1 \\
-2.2 \\
-2.3 \\
-2.4\end{array}$ & $\begin{array}{l}-10.9 \\
-11.7 \\
-12.4 \\
-13.2 \\
-14.0\end{array}$ & $\begin{array}{l}55.6 \\
53.4 \\
51.2 \\
49.0 \\
46.8\end{array}$ & $\begin{array}{l}.0209 \\
.0201 \\
.0193 \\
.0184 \\
.0176\end{array}$ & $\begin{array}{l}.00063 \\
.00061 \\
.00058 \\
.00056 \\
.00053\end{array}$ & $\begin{array}{l}.71 \\
.69 \\
.66 \\
.63 \\
.61\end{array}$ & $\begin{array}{l}.0273 \\
.0273 \\
.0272 \\
.0271 \\
.0271\end{array}$ & $\begin{array}{l}16.86 \\
16.85 \\
16.85 \\
16.85 \\
16.85\end{array}$ \\
\hline $\begin{array}{l}.0 \\
.0 \\
.0 \\
.0 \\
.0\end{array}$ & $\begin{array}{l}-2.5 \\
-2.6 \\
-2.7 \\
-2.8 \\
-2.9\end{array}$ & $\begin{array}{l}-14.9 \\
-15.8 \\
-16.7 \\
-17.7 \\
-18.8\end{array}$ & $\begin{array}{l}44.6 \\
42.4 \\
40.2 \\
38.1 \\
35.9\end{array}$ & $\begin{array}{l}.0168 \\
.0160 \\
.0151 \\
.0143 \\
.0135\end{array}$ & $\begin{array}{l}.00051 \\
.00048 \\
.00046 \\
.00043 \\
.00041\end{array}$ & $\begin{array}{l}.58 \\
.56 \\
.53 \\
.50 \\
.48\end{array}$ & $\begin{array}{l}.0270 \\
.0270 \\
.0269 \\
.0268 \\
.0268\end{array}$ & $\begin{array}{l}16.85 \\
16.85 \\
16.85 \\
16.85 \\
16.85\end{array}$ \\
\hline $\begin{array}{l}.0 \\
.0 \\
.0 \\
.0 \\
.0\end{array}$ & $\begin{array}{l}-3.0 \\
-3.1 \\
-3.2 \\
-3.3 \\
-3.4\end{array}$ & $\begin{array}{l}-19.9 \\
-21.0 \\
-22.2 \\
-23.6 \\
-25.0\end{array}$ & $\begin{array}{l}33.7 \\
31.5 \\
29.4 \\
27.2 \\
25.0\end{array}$ & $\begin{array}{l}.0127 \\
.0119 \\
.0111 \\
.0102 \\
.0094\end{array}$ & $\begin{array}{l}.00039 \\
.00036 \\
.00034 \\
.00031 \\
.00029\end{array}$ & $\begin{array}{l}.45 \\
.42 \\
.40 \\
.37 \\
.34\end{array}$ & $\begin{array}{l}.0267 \\
.0267 \\
.0266 \\
.0265 \\
.0265\end{array}$ & $\begin{array}{l}16.85 \\
16.85 \\
16.85 \\
16.85 \\
16.85\end{array}$ \\
\hline $\begin{array}{l}.0 \\
.0 \\
.0 \\
.0\end{array}$ & $\begin{array}{l}-3.5 \\
-3.6 \\
-3.7 \\
-3.8\end{array}$ & $\begin{array}{l}-26.5 \\
-28.2 \\
-30.0 \\
-32.1\end{array}$ & $\begin{array}{l}22.9 \\
20.7 \\
18.5 \\
16.4\end{array}$ & $\begin{array}{l}.0086 \\
.0078 \\
.0070 \\
.0062\end{array}$ & $\begin{array}{l}.00026 \\
.00024 \\
.00021 \\
.00019\end{array}$ & $\begin{array}{l}.32 \\
.29 \\
.27 \\
.24\end{array}$ & $\begin{array}{l}.0264 \\
.0264 \\
.0263 \\
.0262\end{array}$ & $\begin{array}{l}16.85 \\
16.84 \\
16.84 \\
16.84\end{array}$ \\
\hline
\end{tabular}


$\mathrm{PB}=20.58$, Altitude $=10000-$ Continued

$\begin{array}{lrrrrrrrr}\text { DB } & \text { WB } & \text { DP } & \text { RH } & \text { PV } & \text { W } & \text { H } & \text { S } & \text { V } \\ & & & & & & & & \\ 40.0 & 40.0 & 40.0 & 100.0 & .2477 & .00760 & 17.84 & .0630 & 18.53 \\ 40.0 & 39.0 & 38.2 & 93.2 & .2309 & .00708 & 17.28 & .0619 & 18.52 \\ 40.0 & 38.0 & 36.3 & 86.6 & .2144 & .00657 & 16.73 & .0608 & 18.50 \\ 40.0 & 37.0 & 34.4 & 80.0 & .1982 & .00607 & 16.19 & .0597 & 18.49 \\ 40.0 & 36.0 & 32.3 & 73.6 & .1824 & .00558 & 15.66 & .0586 & 18.47 \\ 40.0 & 35.0 & 30.3 & 67.4 & .1668 & .00510 & 15.14 & .0576 & 18.46 \\ 40.0 & 34.0 & 28.2 & 61.2 & .1516 & .00463 & 14.64 & .0565 & 18.44 \\ 40.0 & 33.0 & 26.0 & 55.1 & .1366 & .00417 & 14.14 & .0555 & 18.43 \\ 40.0 & 32.0 & 24.7 & 52.0 & .1287 & .00393 & 13.88 & .0550 & 18.42 \\ 40.0 & 31.0 & 22.2 & 46.1 & .1142 & .00348 & 13.40 & .0540 & 18.41 \\ 40.0 & 30.0 & 19.4 & 40.4 & .1000 & .00305 & 12.93 & .0530 & 18.40 \\ 40.0 & 29.0 & 16.4 & 34.8 & .0861 & .00262 & 12.47 & .0520 & 18.38 \\ 40.0 & 28.0 & 12.9 & 29.3 & .0725 & .00221 & 12.02 & .0511 & 18.37 \\ 40.0 & 27.0 & 8.8 & 23.9 & .0592 & .00180 & 11.58 & .0501 & 18.36 \\ 40.0 & 26.0 & 3.9 & 18.6 & .0462 & .00140 & 11.16 & .0492 & 18.35 \\ 40.0 & 25.0 & -2.2 & 13.5 & .0334 & .00102 & 10.74 & .0483 & 18.34 \\ 40.0 & 24.0 & -10.9 & 8.5 & .0210 & .00064 & 10.33 & .0474 & 18.33 \\ 40.0 & 23.0 & -26.3 & 3.5 & .0087 & .00026 & 9.93 & .0465 & 18.32\end{array}$


$\mathrm{PB}=20.58$, Altitude $=10000-$ Continued

\begin{tabular}{|c|c|c|c|c|c|c|c|c|}
\hline DB & WB & $\mathrm{DP}$ & $\mathrm{RH}$ & PV & W & $\mathrm{H}$ & $\mathrm{S}$ & V \\
\hline 80.0 & 80.0 & 80.0 & 100.0 & 1.0323 & .03297 & 55.38 & .1349 & 20.82 \\
\hline 80.0 & 79.0 & 78.8 & 96.1 & .9917 & .03161 & 53.89 & .1321 & 20.78 \\
\hline 80.0 & 78.0 & 77.5 & 92.2 & .9522 & .03028 & 52.44 & .1294 & 20.74 \\
\hline 80.0 & 77.0 & 76.3 & 88.5 & .9135 & .02899 & 51.03 & .1268 & 20.70 \\
\hline 80.0 & 76.0 & 75.0 & 84.8 & .8757 & .02774 & 49.65 & .1242 & 20.66 \\
\hline 80.0 & 75.0 & 73.7 & 81.2 & .8387 & .02652 & 48.31 & .1217 & 20.62 \\
\hline 80.0 & 74.0 & 72.4 & 77.8 & .8026 & .02533 & 47.01 & .1192 & 20.58 \\
\hline 80.0 & 73.0 & 71.1 & 74.3 & .7673 & .02417 & 45.74 & .1169 & 20.54 \\
\hline 80.0 & 72.0 & 69.8 & 71.0 & .7329 & .02305 & 44.51 & .1145 & 20.51 \\
\hline 80.0 & 71.0 & 68.4 & 67.7 & .6992 & .02195 & 43.31 & .1122 & 20.47 \\
\hline 80.0 & 70.0 & 67.0 & 64.5 & .6663 & .02088 & 42.14 & .1100 & 20.44 \\
\hline 80.0 & 69.0 & 65.5 & 61.4 & .6341 & .01984 & 41.00 & .1079 & 20.41 \\
\hline 80.0 & 68.0 & 64.1 & 58.4 & .6027 & .01883 & 39.89 & .1057 & 20.37 \\
\hline 80.0 & 67.0 & 62.6 & 55.4 & .5719 & .01784 & 38.80 & .1037 & 20.34 \\
\hline 80.0 & 66.0 & 61.1 & 52.5 & .5419 & .01688 & 37.75 & .1016 & 20.31 \\
\hline 80.0 & 65.0 & 59.5 & 49.7 & .5126 & .01594 & 36.72 & .0997 & 20.28 \\
\hline 80.0 & 64.0 & 57.9 & 46.9 & .4839 & .01503 & 35.72 & .0977 & 20.25 \\
\hline 80.0 & 63.0 & 56.2 & 44.2 & .4558 & .01413 & 34.74 & .0958 & 20.22 \\
\hline 80.0 & 62.0 & 54.5 & 41.5 & .4284 & .01327 & 33.79 & .0940 & 20.20 \\
\hline 80.0 & 61.0 & 52.8 & 38.9 & .4016 & .01242 & 32.86 & .0922 & 20.17 \\
\hline 80.0 & 60.0 & 50.9 & 36.4 & .3754 & .01159 & 31.96 & .0904 & 20.14 \\
\hline 80.0 & 59.0 & 49.0 & 33.9 & .3497 & .01079 & 31.07 & .0887 & 20.12 \\
\hline 80.0 & 58.0 & 47.1 & 31.5 & .3247 & .01000 & 30.21 & .0870 & 20.09 \\
\hline 80.0 & 57.0 & 45.0 & 29.1 & .3002 & .00924 & 29.37 & .0853 & 20.07 \\
\hline 80.0 & 56.0 & 42.8 & 26.8 & .2762 & .00849 & 28.55 & .0837 & 20.05 \\
\hline 80.0 & 55.0 & 40.5 & 24.5 & .2527 & .00776 & 27.75 & .0821 & 20.02 \\
\hline 80.0 & 54.0 & 38.1 & 22.3 & .2298 & .00705 & 26.97 & .0805 & 20.00 \\
\hline 80.0 & 53.0 & 35.5 & 20.1 & .2073 & .00635 & 26.21 & .0790 & 19.98 \\
\hline 80.0 & 52.0 & 32.7 & 18.0 & .1854 & .00567 & 25.47 & .0775 & 19.96 \\
\hline 80.0 & 51.0 & 29.9 & 15.9 & .1639 & .00501 & 24.74 & .0760 & 19.94 \\
\hline 80.0 & 50.0 & 27.0 & 13.8 & .1429 & .00436 & 24.03 & .0746 & 19.91 \\
\hline 80.0 & 49.0 & 23.7 & 11.8 & .1223 & .00373 & 23.34 & .0731 & 19.89 \\
\hline 80.0 & 48.0 & 19.9 & 9.9 & .1021 & .00311 & 22.66 & .0717 & 19.87 \\
\hline 80.0 & 47.0 & 15.5 & 8.0 & .0824 & .00251 & 22.00 & .0703 & 19.86 \\
\hline 80.0 & 46.0 & 10.1 & 6.1 & .0631 & .00192 & 21.35 & .0690 & 19.84 \\
\hline 80.0 & 45.0 & 3.1 & 4.3 & .0442 & .00134 & 20.72 & .0676 & 19.82 \\
\hline 80.0 & 44.0 & -7.2 & 2.5 & .0257 & .00078 & 20.10 & .0662 & 19.80 \\
\hline 80.0 & 43.0 & -28.7 & .7 & .0076 & .00023 & 19.50 & .0649 & 19.78 \\
\hline
\end{tabular}




$$
\mathrm{PB}=20.58, \text { Altitude }=10000-\text { Continued }
$$

\begin{tabular}{|c|c|c|c|c|c|c|c|c|}
\hline $\mathrm{DB}$ & WB & $\mathrm{DP}$ & $\mathrm{RH}$ & PV & W & $\mathrm{H}$ & S & V \\
\hline $\begin{array}{l}120.0 \\
120.0 \\
120.0 \\
120.0 \\
120.0\end{array}$ & $\begin{array}{l}120.0 \\
118.0 \\
116.0 \\
114.0 \\
112.0\end{array}$ & $\begin{array}{l}120.0 \\
117.9 \\
115.7 \\
113.5 \\
111.3\end{array}$ & $\begin{array}{r}100.0 \\
94.2 \\
88.6 \\
83.3 \\
78.2\end{array}$ & $\begin{array}{l}3.4476 \\
3.2472 \\
3.0553 \\
2.8717 \\
2.6961\end{array}$ & $\begin{array}{l}.12583 \\
.11713 \\
.10899 \\
.10137 \\
.09422\end{array}$ & $\begin{array}{l}168.92 \\
159.25 \\
150.19 \\
141.71 \\
133.77\end{array}$ & $\begin{array}{l}.3373 \\
.3204 \\
.3046 \\
.2898 \\
.2758\end{array}$ & $\begin{array}{l}25.52 \\
25.23 \\
24.95 \\
24.69 \\
24.45\end{array}$ \\
\hline $\begin{array}{l}120.0 \\
120.0 \\
120.0 \\
120.0 \\
120.0\end{array}$ & $\begin{array}{l}110.0 \\
108.0 \\
106.0 \\
104.0 \\
102.0\end{array}$ & $\begin{array}{r}109.1 \\
106.8 \\
104.5 \\
102.2 \\
99.9\end{array}$ & $\begin{array}{l}73.3 \\
68.7 \\
64.2 \\
60.0 \\
55.9\end{array}$ & $\begin{array}{l}2.5281 \\
2.3675 \\
2.2139 \\
2.0671 \\
1.9268\end{array}$ & $\begin{array}{l}.08752 \\
.08123 \\
.07532 \\
.06976 \\
.06453\end{array}$ & $\begin{array}{l}126.31 \\
119.31 \\
112.73 \\
106.54 \\
100.72\end{array}$ & $\begin{array}{l}.2627 \\
.2503 \\
.2386 \\
.2275 \\
.2171\end{array}$ & $\begin{array}{l}24.22 \\
24.01 \\
23.81 \\
23.62 \\
23.44\end{array}$ \\
\hline $\begin{array}{l}120.0 \\
120.0 \\
120.0 \\
120.0 \\
120.0\end{array}$ & $\begin{array}{r}100.0 \\
98.0 \\
96.0 \\
94.0 \\
92.0\end{array}$ & $\begin{array}{l}97.5 \\
95.1 \\
92.6 \\
90.1 \\
87.5\end{array}$ & $\begin{array}{l}52.0 \\
48.3 \\
44.7 \\
41.3 \\
38.1\end{array}$ & $\begin{array}{l}1.7928 \\
1.6647 \\
1.5424 \\
1.4256 \\
1.3140\end{array}$ & $\begin{array}{l}.05961 \\
.05497 \\
.05060 \\
.04648 \\
.04259\end{array}$ & $\begin{array}{l}95.25 \\
90.08 \\
85.22 \\
80.63 \\
76.30\end{array}$ & $\begin{array}{l}.2073 \\
.1979 \\
.1891 \\
.1808 \\
.1729\end{array}$ & $\begin{array}{l}23.27 \\
23.12 \\
22.97 \\
22.83 \\
22.70\end{array}$ \\
\hline $\begin{array}{l}120.0 \\
120.0 \\
120.0 \\
120.0 \\
120.0\end{array}$ & $\begin{array}{l}90.0 \\
88.0 \\
86.0 \\
84.0 \\
82.0\end{array}$ & $\begin{array}{l}84.8 \\
82.1 \\
79.3 \\
76.4 \\
73.3\end{array}$ & $\begin{array}{l}35.0 \\
32.1 \\
29.3 \\
26.6 \\
24.0\end{array}$ & $\begin{array}{r}1.2075 \\
1.1058 \\
1.0088 \\
.9161 \\
.8277\end{array}$ & $\begin{array}{l}.03892 \\
.03545 \\
.03218 \\
.02908 \\
.02616\end{array}$ & $\begin{array}{l}72.21 \\
68.35 \\
64.71 \\
61.26 \\
58.00\end{array}$ & $\begin{array}{l}.1654 \\
.1582 \\
.1515 \\
.1451 \\
.1390\end{array}$ & $\begin{array}{l}22.57 \\
22.45 \\
22.34 \\
22.24 \\
22.14\end{array}$ \\
\hline $\begin{array}{l}120.0 \\
120.0 \\
120.0 \\
120.0 \\
120.0\end{array}$ & $\begin{array}{l}80.0 \\
78.0 \\
76.0 \\
74.0 \\
72.0\end{array}$ & $\begin{array}{l}70.2 \\
66.8 \\
63.3 \\
59.5 \\
55.4\end{array}$ & $\begin{array}{l}21.6 \\
19.2 \\
17.0 \\
14.9 \\
12.8\end{array}$ & $\begin{array}{l}.7433 \\
.6627 \\
.5858 \\
.5124 \\
.4424\end{array}$ & $\begin{array}{l}.02339 \\
.02077 \\
.01829 \\
.01594 \\
.01371\end{array}$ & $\begin{array}{l}54.92 \\
52.00 \\
49.23 \\
46.62 \\
44.14\end{array}$ & $\begin{array}{l}.1332 \\
.1276 \\
.1224 \\
.1173 \\
.1126\end{array}$ & $\begin{array}{l}22.04 \\
21.95 \\
21.87 \\
21.79 \\
21.71\end{array}$ \\
\hline $\begin{array}{l}120.0 \\
120.0 \\
120.0 \\
120.0 \\
120.0\end{array}$ & $\begin{array}{l}70.0 \\
68.0 \\
66.0 \\
64.0 \\
62.0\end{array}$ & $\begin{array}{l}51.0 \\
46.0 \\
40.3 \\
33.6 \\
26.0\end{array}$ & $\begin{array}{r}10.9 \\
9.0 \\
7.3 \\
5.6 \\
4.0\end{array}$ & $\begin{array}{l}.3755 \\
.3116 \\
.2507 \\
.1925 \\
.1368\end{array}$ & $\begin{array}{l}.01160 \\
.00959 \\
.00769 \\
.00589 \\
.00418\end{array}$ & $\begin{array}{l}41.78 \\
39.55 \\
37.44 \\
35.43 \\
33.52\end{array}$ & $\begin{array}{l}.1080 \\
.1036 \\
.0994 \\
.0954 \\
.0915\end{array}$ & $\begin{array}{l}21.64 \\
21.57 \\
21.51 \\
21.45 \\
21.39\end{array}$ \\
\hline $\begin{array}{l}120.0 \\
120.0\end{array}$ & $\begin{array}{l}60.0 \\
58.0\end{array}$ & $\begin{array}{r}15.8 \\
-2.5\end{array}$ & $\begin{array}{l}2.4 \\
1.0\end{array}$ & $\begin{array}{l}.0837 \\
.0329\end{array}$ & $\begin{array}{l}.00255 \\
.00100\end{array}$ & $\begin{array}{l}31.70 \\
29.98\end{array}$ & $\begin{array}{l}.0877 \\
.0840\end{array}$ & $\begin{array}{l}21.33 \\
21.28\end{array}$ \\
\hline
\end{tabular}


$\mathrm{PB}=20.58$, Altitude $=10000-$ Continued

\begin{tabular}{|c|c|c|c|c|c|c|c|c|}
\hline $\mathrm{DB}$ & WB & $\mathrm{DP}$ & $\mathrm{RH}$ & PV & W & $\mathrm{H}$ & $\mathrm{S}$ & V \\
\hline 160.0 & 160.0 & 160.0 & 100.0 & 9.6555 & .55435 & 664.78 & 1.1654 & 42.79 \\
\hline 160.0 & 158.0 & 158.0 & 95.2 & 9.1946 & .50641 & 610.68 & 1.0768 & 41.06 \\
\hline 160.0 & 156.0 & 155.9 & 90.6 & 8.7510 & .46382 & 562.61 & .9978 & 39.52 \\
\hline 160.0 & 154.0 & 153.9 & 86.2 & 8.3242 & .42575 & 519.65 & .9270 & 38.15 \\
\hline 160.0 & 152.0 & 151.8 & 82.0 & 7.9138 & .39157 & 481.06 & .8632 & 36.91 \\
\hline 160.0 & 150.0 & 149.7 & 77.9 & 7.5192 & .36074 & 446.25 & .8055 & 35.80 \\
\hline 160.0 & 148.0 & 147.6 & 73.9 & 7.1399 & .33282 & 414.72 & .7531 & 34.79 \\
\hline 160.0 & 146.0 & 145.5 & 70.2 & 6.7754 & .30744 & 386.06 & .7053 & 33.87 \\
\hline 160.0 & 144.0 & 143.4 & 66.5 & 6.4253 & .28429 & 359.92 & .6616 & 33.03 \\
\hline 160.0 & 142.0 & 141.3 & 63.1 & 6.0891 & .26312 & 336.00 & .6215 & 32.27 \\
\hline 160.0 & 140.0 & 139.2 & 59.7 & 5.7663 & .24370 & 314.07 & .5846 & 31.56 \\
\hline 160.0 & 138.0 & 137.1 & 56.5 & 5.4565 & .22585 & 293.89 & .5505 & 30.92 \\
\hline 160.0 & 136.0 & 134.9 & 53.4 & 5.1592 & .20939 & 275.30 & .5190 & 30.32 \\
\hline 160.0 & 134.0 & 132.8 & 50.5 & 4.8740 & .19420 & 258.13 & .4899 & 29.77 \\
\hline 160.0 & 132.0 & 130.6 & 47.6 & 4.6005 & .18014 & 242.24 & .4628 & 29.26 \\
\hline 160.0 & 130.0 & 128.4 & 44.9 & 4.3383 & .16711 & 227.51 & .4376 & 28.79 \\
\hline 160.0 & 128.0 & 126.2 & 42.3 & 4.0870 & .15500 & 213.83 & .4141 & 28.35 \\
\hline 160.0 & 126.0 & 124.0 & 39.8 & 3.8462 & .14375 & 201.11 & .3922 & 27.94 \\
\hline 160.0 & 124.0 & 121.7 & 37.4 & 3.6155 & .13328 & 189.27 & .3717 & 27.56 \\
\hline 160.0 & 122.0 & 119.4 & 35.2 & 3.3946 & .12351 & 178.22 & .3526 & 27.21 \\
\hline 160.0 & 120.0 & 117.1 & 33.0 & 3.1830 & .11439 & 167.91 & .3347 & 26.87 \\
\hline 160.0 & 118.0 & 114.8 & 30.9 & 2.9805 & 10587 & 158.28 & .3178 & 26.57 \\
\hline 160.0 & 116.0 & 112.5 & 28.9 & 2.7868 & .09789 & 149.26 & .3020 & 26.28 \\
\hline 160.0 & 114.0 & 110.1 & 26.9 & 2.6014 & .09043 & 140.82 & .2872 & 26.00 \\
\hline 160.0 & 112.0 & 107.6 & 25.1 & 2.4240 & .08343 & 132.90 & .2732 & 25.75 \\
\hline 160.0 & 110.0 & 105.2 & 23.3 & 2.2544 & .07687 & 125.48 & .2600 & 25.51 \\
\hline 160.0 & 108.0 & 102.6 & 21.7 & 2.0923 & .07071 & 118.51 & .2476 & 25.29 \\
\hline 160.0 & 106.0 & 100.1 & 20.1 & 1.9373 & .06492 & 111.96 & .2359 & 25.08 \\
\hline 160.0 & 104.0 & 97.4 & 18.5 & 1.7892 & .05948 & 105.80 & .2249 & 24.88 \\
\hline 160.0 & 102.0 & 94.7 & 17.1 & 1.6477 & .05436 & 100.01 & .2144 & 24.69 \\
\hline 160.0 & 100.0 & 92.0 & 15.7 & 1.5125 & .04954 & 94.56 & .2045 & 24.52 \\
\hline 160.0 & 98.0 & 89.1 & 14.3 & 1.3833 & .04500 & 89.42 & .1952 & 24.35 \\
\hline 160.0 & 96.0 & 86.2 & 13.0 & 1.2600 & .04072 & 84.59 & .1863 & 24.20 \\
\hline 160.0 & 94.0 & 83.1 & 11.8 & 1.1423 & .03669 & 80.02 & .1780 & 24.05 \\
\hline 160.0 & 92.0 & 79.9 & 10.7 & 1.0299 & .03289 & 75.72 & .1700 & 23.91 \\
\hline 160.0 & 90.0 & 76.6 & 9.6 & .9226 & .02930 & 71.66 & .1625 & 23.78 \\
\hline 160.0 & 88.0 & 73.1 & 8.5 & .8202 & .02591 & 67.82 & .1553 & 23.66 \\
\hline 160.0 & 86.0 & 69.3 & 7.5 & .7224 & .02271 & 64.20 & .1485 & 23.54 \\
\hline 160.0 & 84.0 & 65.3 & 6.5 & .6292 & .01968 & 60.77 & .1420 & 23.43 \\
\hline 160.0 & 82.0 & 61.0 & 5.6 & .5402 & .01682 & 57.53 & .1358 & 23.33 \\
\hline 160.0 & 80.0 & 56.2 & 4.7 & .4553 & .01412 & 54.47 & .1299 & 23.23 \\
\hline 160.0 & 78.0 & 50.9 & 3.9 & .3742 & .01156 & 51.57 & .1243 & 23.13 \\
\hline 160.0 & 76.0 & 44.7 & 3.1 & .2969 & .00913 & 48.83 & .1189 & 23.05 \\
\hline 160.0 & 74.0 & 37.3 & 2.3 & .2232 & .00684 & 46.23 & .1138 & 22.96 \\
\hline 160.0 & 72.0 & 28.4 & 1.6 & .1528 & .00467 & 43.77 & .1088 & 22.88 \\
\hline 160.0 & 70.0 & 16.2 & .9 & .0856 & .00261 & 41.44 & .1040 & 22.81 \\
\hline 160.0 & 68.0 & -10.4 & .2 & .0215 & .00065 & 39.23 & .0992 & 22.74 \\
\hline
\end{tabular}




\section{Announcement of New Publications in Building Science Series}

\section{Superintendent of Documents,}

Government Printing Office,

Washington, D.C. 20402

\section{Dear Sir:}

Please add my name to the announcement list of new publications to be issued in the series: National Bureau of Standards Building Science Series.

Name

Company

Address

City State Zip Code

(Notification key N-339) 



\section{NATIONAL BUREAU OF STANDARDS}

The National Bureau of Standards ' was established by an act of Congress March 3, 1901. Today, in addition to serving as the Nation's central measurement laboratory, the Burcau is a principal focal point in the Federal Government for assuring maximum application of the physical and engineering sciences to the advancement of tcchnology in industry and commercc. To this cnd the Bureau conducts research and provides central national scrvices in four broad program areas. These are: (1) basic measurcments and standards, (2) matcrials measurcments and standards, (3) technological measurements and standards, and (4) transfer of tcchnology.

The Bureau comprises the Institute for Basic Standards, the Institute for Materials Rcscarch, the Institute for Applied Technology, the Center for Radiation Research, the Center for Computer Sciences and Technology, and the Office for Information Programs.

THE INSTITUTE FOR BASIC STANDARDS provides the central basis within the United States of a complete and consistent system of physical measurement; coordinates that system with measurement systems of other nations; and furnishes essential services leading to accurate and uniform physical measurements throughout the Nation's scientific community, industry, and commerce. The Institute consists of an Office of Measurement Services and the following technical divisions:

Applied Mathematics-Electricity-Metrology-Mechanics-Hcat-Atomic and Molecular Physics_Radio Physics "-Radio Engineering "-Time and Frequency -_Astrophysics $=$-Cryogenics."

THE INSTITUTE FOR MATERIALS RESEA RCH conducts materials research leading to improved methods of measurement standards, and data on the properties of well-characterized materials needed by industry, commerce, educational institutions, and Government; develops, produces, and distributes standard reference materials; relates the physical and chemical properties of materials to their behavior and their interaction with their environments; and provides advisory and research services to other Government agencies. The Institute consists of an Office of Standard Reference Materials and the following divisions:

Analytical Chemistry_Polymers-Metallurgy_Inorganic Materials-Physical Chemistry. THE INSTITUTE FOR APPLIED TECHNOLOGY provides technical services to promote the use of available technology and to facilitate technological innovation in industry and Government; cooperates with public and private organizations in the development of technological standards, and test methodologies; and provides advisory and research services for Federal, state, and local government agencies. The Institute consists of the following technical divisions and offices:

Engineering Standards-Weights and Measures - Invention and Innovation - Vehicle Systems Research-Product Evaluation-Building Research-Instrument Shops-Measurement Engineering-Electronic Technology_Technical Analysis.

THE CENTER FOR RADIATION RESEARCH engages in research, measurement, and application of radiation to the solution of Bureau mission problems and the problems of other agencies and institutions. The Center consists of the following divisions:

Reactor Radiation-Linac Radiation-Nuclear Radiation-Applied Radiation.

THE CENTER FOR COMPUTER SCIENCES AND TECHNOLOGY conducts research and provides technical services designed to aid Government agencies in the selection, acquisition, and effective use of automatic data processing equipment; and serves as the principal focus for the development of Federal standards for automatic data processing equipment, techniques, and computer languages. The Center consists of the following offices and divisions:

Information Processing Standards-Computer Information - Computer Services - Systems Development-Information Processing Technology.

THE OFFICE FOR INFORMATION PROGRAMS promotes optimum dissemination and accessibility of scientific information generated within NBS and other agencies of the Federal government; promotes the development of the National Standard Reference Data System and a system of information analysis centers dealing with the broader aspects of the National Measurement System, and provides appropriate services to ensure that the NBS staff has optimum accessibility to the scientific information of the world. The Office consists of the following organizational units:

Office of Standard Reference Data-Clearinghouse for Federal Scientific and Technical Information "-Office of Technical Information and Publications-Library-Office of Public Information-Office of International Relations.

\footnotetext{
Headquarters and Laboratories at Gaithersburg. Maryland, unless otherwise noted; mailing address Washington, D.C. 20234.

Located at Boulder. Colorado 80302 .

is I.ocated at 5285 Port Royal Road, Springfield, Virginia 22151.
} 
U.S. DEPARTMENT OF COMMERCE

Washington, D.C. 20230

OFFICIAL BUSINESS

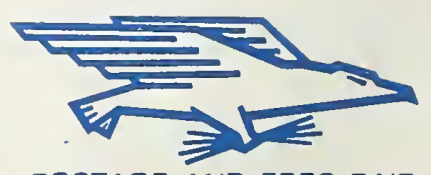

POSTAGE AND FEES PAID U.S. DEPARTMENT OF COMMERCE 